\title{
Kinematic Calibration of a
}

\section{Reconfigurable Robot (RoboTwin)}

by

Nitin Juneja

\author{
A Thesis \\ Submitted in Partial Fulfillment of the Requirements \\ for the Degree of Master of Applied Science \\ in the \\ University of Toronto \\ copyright Nitin Juneja, 1996
}

Department of Mechanical Enginnering

University of Toronto

January 1996 
National Library

of Canada

Acquisitions and

Bibliographic Services

395 Wellington Street

Ottawa ON KIA ONA

Canada
Bibliothèque nationale du Canada

Acquisitions et services bibliographiques

395. nue Wellington Ottawa ON KIA ONY4

Canada
The author has granted a nonexclusive licence allowing the National Library of Canada to reproduce, loan, distribute or sell copies of this thesis in microform, paper or electronic formats.

The author retains ownership of the copyright in this thesis. Neither the thesis nor substantial extracts from it may be printed or otherwise reproduced without the author's permission.
L'auteur a accordé une licence non exclusive permettant à la Bibliothèque nationale du Canada de reproduire, prêter, distribuer ou vendre des copies de cette thèse sous la forme de microfiche/film, de reproduction sur papier ou sur format électronique.

L'auteur conserve la propriété du droit d'auteur qui protège cette thèse. Ni la thèse ni des extraits substantiels de celle-ci ne doivent être imprimés ou autrement reproduits sans son autorisation. 


\section{Acknowledgments}

I would like to thank Professor A.A. Goldenberg for giving me the opportunity to work with him and learn from him. His help in writing this thesis is invaluable.

I would also like to thank Dr. M. Kircanski who was always there for critical discussions.

I am grateful to Institute for Robotics and Intelligent Systems (IRIS) for the financial support to this project.

I would also like to thank my colleagues in Robotics and Automation Lab (RAL) for their support and help. 


\section{Abstract}

Kinematic calibration is the process of determination of actual robot parameters using the nominal geometric parameters of the robot and specific measurements made on the robot. Periodic kinematic calibration of a robot is important to maintain its end point accuracy in the presence of wear. For a new robot, kinematic calibration is essential for improving the end point accuracy. For a robot programmed off-line, a calibration methodology which can be used easily at regular intervals, is necessary.

The RoboTwin is a modular, expandable and re-configurable robot system, designed and patented by Robotics and Automation Lab (RAL), University of Toronto. It can be configured in one of many configurations. Kinematic calibration of such a system possesses a new challenge as the calibration methodology has to be generic enough to be configuration independent. This thesis presents a calibration methodology for a re-configurable robot system (RoboTwin).

In general, calibration consists of four phases: modelling, measurement, identification and implementation. Modelling deals with development of a kinematic model representing the end point position as a function of the robot parameters. This is done by making use of homogeneous transformations and a modified Denavit Hartenberg notation. Measurement deals with collecting end-point pose data by using a new 6-dof ball bar mechanism. The data collected is used in the 
identification phase. The identification phase determines the corrected parameters of the robot parameters based on the data collected during the measurement phase. The identification algorithm used is based on a linear least squares method. To remove the error due to linearization of the identification problem, the least squares method is used iteratively. The implementation phase deals with issues related to using the corrected robot parameters in the robot's controller. Implementation is not dealt with in this thesis, as the RoboTwin is currently used as a test bed for controllers and does not have one controller.

The calibration methodology developed is implemented on the RoboTwin. A user interface is developed to guide the user through the task of calibration. The results of calibration for two RoboTwin configurations are presented. 


\section{Table of Contents}

Acknowledgments

Abstract

Table of Contents

List of Figures

List of Tables

Chapter 1 :Introduction

1.1 What is Calibration?

1.2 Motivation

1.3 Layout of the Thesis

1.4 Contributions of the Thesis

2.1 Why is Kinematic Calibration necessary? 
2.2 How is Kinematic Calibration achieved?

2.2.1 Modelling

2.2.2 Measurement

22

2.2.3 Identification

2.2.4 Implementation

29

Chapter 3 :An Algorithm for RoboTwin Calibration

30

3.1 The RoboTwin: A modular, re-configurable and expandable robot 30

3.1.1 Mechanical Structure 30

3.1.2 Control Architecture

3.2 Design Criterion 34

3.3 An Algorithm for Calibration 38

3.3.1 Modelling 38

3.3.1.1 The DH Model 39

3.3.1.2 Hayati-DH Model for Near-Parallel Rotary Joints 42 3.3.1.3 A Model for RoboTwin 
Chapter 4 :Implementation of the Calibration Algorithm and Analysis of Results

4.1 Implementation on a DH configuration

4.2 Implementation on a Hayati Configuration

4.3 Analysis of Results

Chapter 5 :Conclusions and Future Work

5.1 Future Work

References 


\section{List of Figures}

Figure 1. Dynamic and Static Calibration ...................................................................... 13

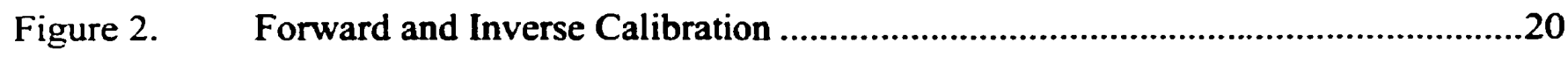

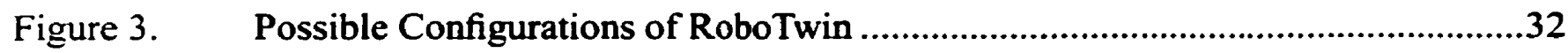

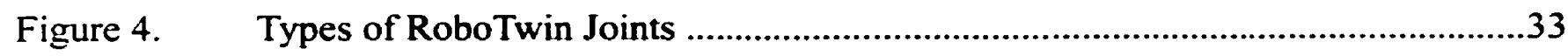

Figure 5. Schematic Diagram of Robot Joints ................................................................34

Figure 6. Schematic Diagram of Re-configurable Joints ….................................................35

Figure 7. Base-line Configuration of RoboTwin ..............................................................36

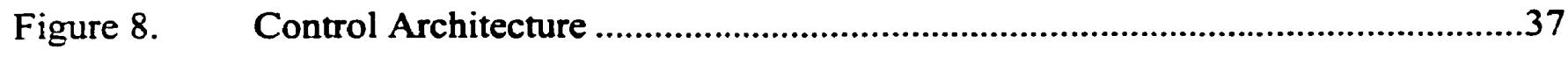

Figure 9. Labelling and Allocation of Frames in an open loop kinematic chain ..................40

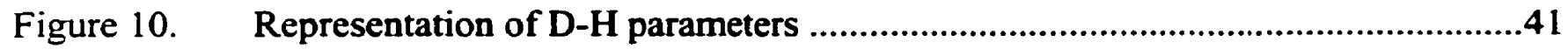

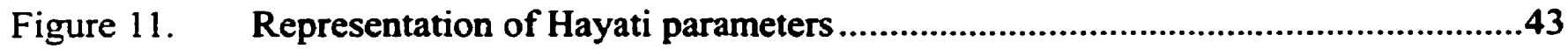

Figure 12. Mobile Closed Kinematic Chain........................................................................48 


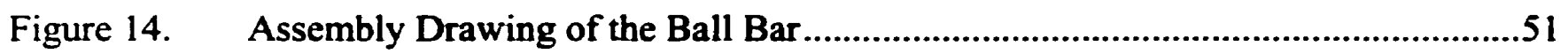

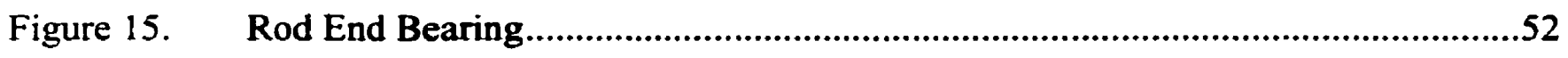

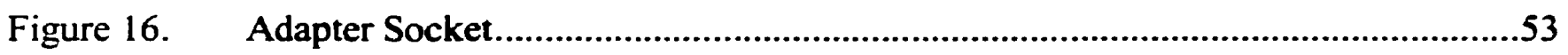

Figure 17. Workspace covered by the Ball-bar mechanism ...........................................54

Figure 18. Vector Representation of the Ball-bar in base frame .......................................55

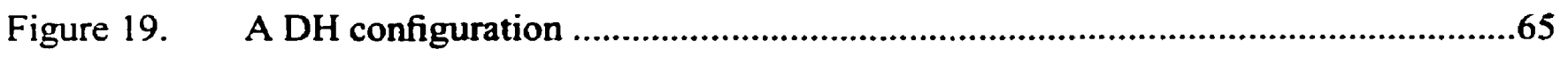

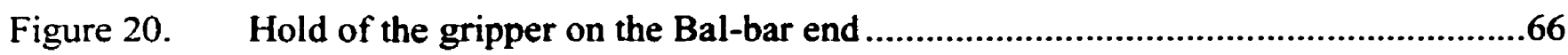

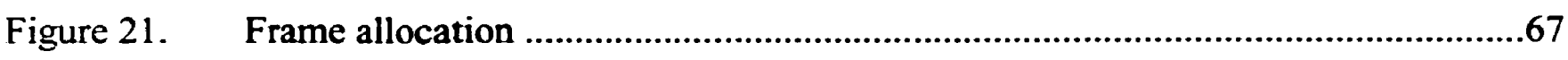

Figure 22. Error in the Bar Length (Identification Using data set 1) .............................72

Figure 23. Error Frequency Distribution (Identification using data set 1) .......................72

Figure 24. Error in Bar length (Identification Using data set 2) ..................................73

Figure 25. Error Frequency Distribution (Identification Using data set 2) ......................73

Figure 26. Error in bar length (Identification Using data set 3) ..................................74

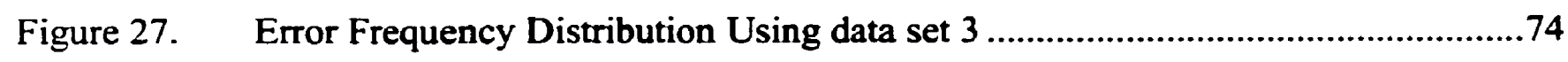

Figure 28. Error in Bar Length (Identification Using the complete data set)......................75

Figure 29. Error Frequency Distribution (Identification Using the Complete Data Set) ........75

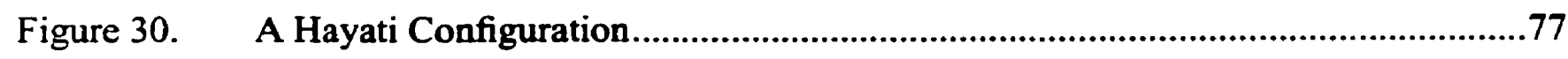


Figure 31. Frame Allocation for the Hayati Configuration .............................................78

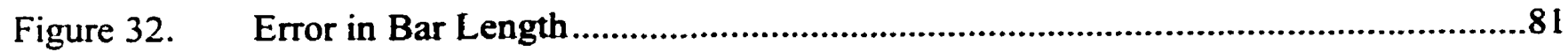

Figure 33. Frequency Distribution of Error in Bar Length .......................................81 


\section{List of Tables}

TABLE I. Nominal Measurements of the Robot .......................................................68

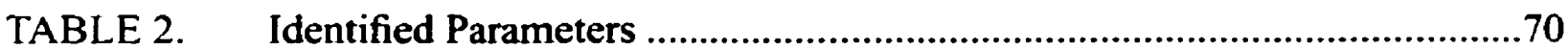

TABLE 3. Mean and SD of bar length error using identified parameters ....................76

TABLE 4. Mean and SD of the bar error using nominal parameters ..........................76

TABLE 5. Nominal parameters for Hayati configuration ..........................................78

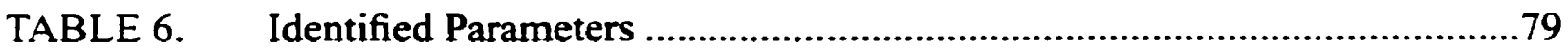

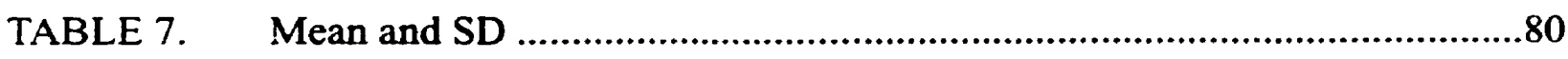




\section{Chapter 1}

\section{Introduction}

High accuracy is critical for a robot to be used in automated flexible manufacturing. Unfortunately, most of industrial manipulators are unable to reach a point in the workspace with a very high accuracy.

A manufactured robot is usually not accurate due to assembly errors and manufacturing limitations. The accuracy of the end-point can be improved by manufacturing the components with tighter tolerances and improving the manufacturing methods. However, this is capital intensive. A low cost alternative of improving accuracy is kinematic calibration of the robot.

\subsection{What is Calibration?}

Designers describe the robot by means of parameters representing the geometry (link lengths, link orientations) and the mechanical characteristics (link masses, link stiffness). Robot calibration is a term applied to the procedures used in determining the 'actual' parameters which describe the geometrical dimensions and mechanical characteristics of the manufactured robot structure. 
The parameters describing a robot structure may be kinematic or dynamic parameters. Based on the parameters, robot calibration may either be kinematic calibration or dynamic calibration (figure 1). Kinematic calibration involves identification of those parameters which influence the static characteristics of the robot (kinematic parameters). These parameters include link lengths, relative joint axis orientations, actuator/link elasticity. Dynamic calibration involves identification of parameters affecting the motion characteristics of the robot (dynamic parameters). These parameters include friction, masses, inertias, stiffness and elasticity.

The accuracy of the end-point at steady state is affected by the kinematic parameters. The precise knowledge of kinematic parameters of a robot is useful for improving the end-point accuracy of the robot. Kinematic calibration can also be defined as a means of improving the accuracy of the end-point of the robot by identifying the kinematic parameters of a robot more accurately and making changes in the positioning software. This thesis deals with the kinematic calibration of a re-configurable robot system (RoboTwin).

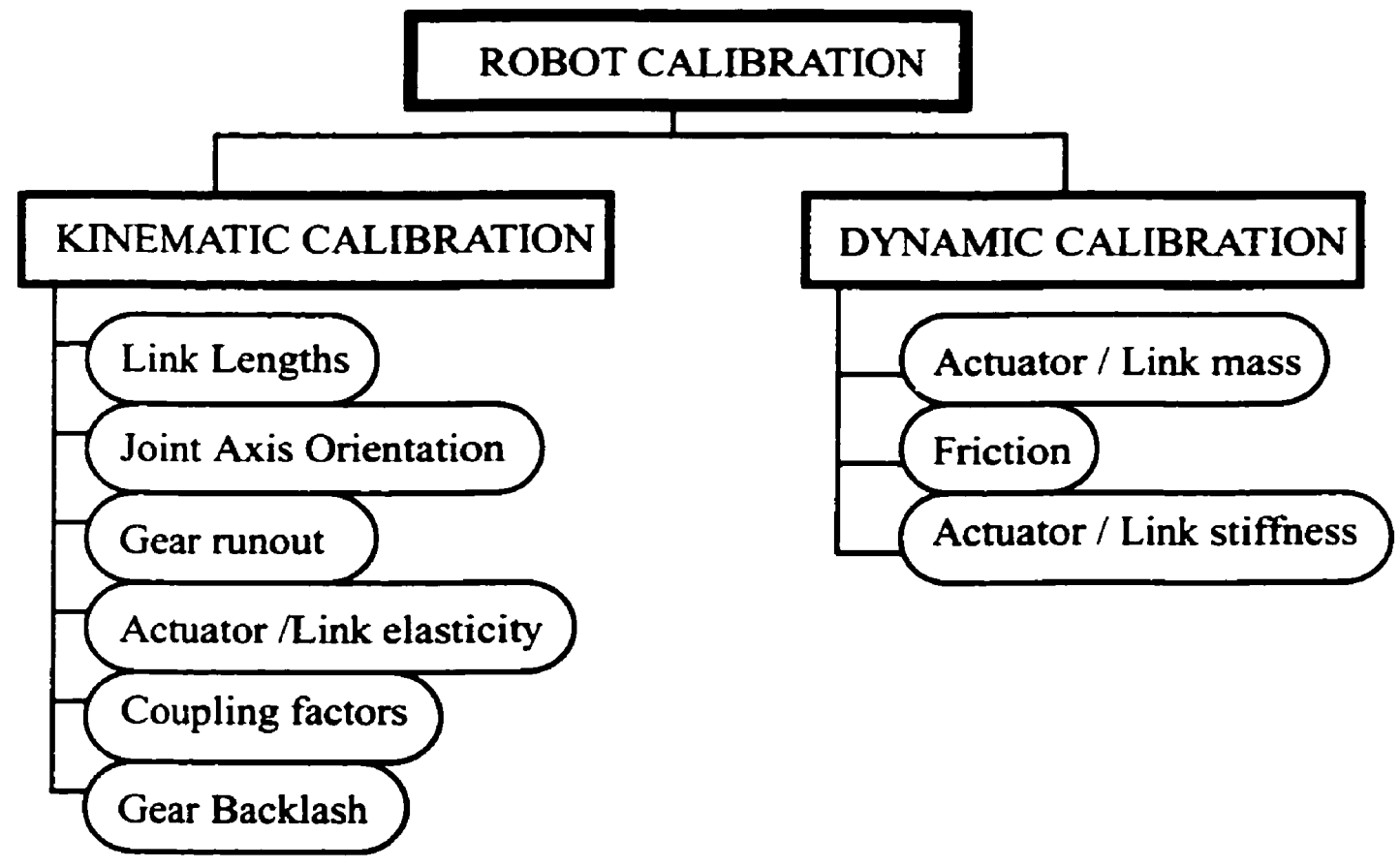

Figure 1. Dynamic and Static Calibration 
The differences between a real robot and its 'nominal robot' (as described by the designer) arise due to many factors. These factors can be classified as geometrical or non-geometrical factors. Geometrical errors (caused due to geometrical factors) include errors due to manufacturing tolerances during manufacturing and mounting errors during robot assembly. Nongeometrical errors include link and joint flexibility, backlash, gear transmission errors and errors due to temperature effects.

Both geometrical and non-geometrical errors affect the end-point accuracy achieved by the robot. However, all non-geometrical errors account for only $10 \%$ of the total end-point error [1].

\subsection{Motivation}

The RoboTwin is a modular, reconfigurable and expandable system developed and patented by the Robotics and Automation Lab (RAL), University of Toronto, Canada. Kinematic calibration is essential for improving the accuracy of such a system. The reconfigurability of the RoboTwin adds a new challenge in developing a calibration system that is generic enough to accommodate the wide range of configurations that the system can be assembled into.

This thesis develops a calibration methodology for calibrating the RoboTwin to achieve a significant improvement in the end-point accuracy.

\subsection{Layout of the Thesis}

In chapter 2 the why and how of kinematic calibration is explained. The question: Why is kinematic calibration necessary? is answered in section 2.1 . In section 2.2 , the methodology to calibrate a robot is explained by classifying calibration in four stages of Modelling, Measurement, 
Identification and Implementation. Previous work in the field of calibration is presented as it relates to the four stages of calibration.

In chapter 3, the RoboTwin is described and an algorithm for calibration of the RoboTwin is developed. In section 3.1, the mechanical structure and control architecture of the RoboTwin is described. In section 3.2, a design criterion for designing a calibration system is established. An algorithm for calibration the RoboTwin is developed in section 3.3. The modelling methodology is developed in section 3.3.1; the measurement methodology is developed in section 3.3.2; and the identification methodology is developed in section 3.3.3. A summary of the algorithm is presented in 3.3.4. A description of the user-interface is presented in section 3.3.5.

In chapter 4 , the algorithm is implemented on two RoboTwin configurations. The results of calibration are presented in sections 4.1 and 4.2. The results of calibration are analyzed in section 4.3.

In chapter 5, conclusions are presented and future work is suggested.

\subsection{Contributions of the Thesis}

A calibration methodology for calibrating the RoboTwin was developed and implemented, leading to a $90 \%$ improvement in the accuracy of the robot. A user-interface was designed for guiding the user through the task of calibration.

A new passive measurement scheme was developed using a flexible 6-dof ball bar mechanism which made it possible to collect partial pose data over the entire workspace of the robot.

An identification algorithm using the least square method was implemented. The identification was achieved by using the length of the ball-bar mechanism as a 'yardstick'. The 
length of the bar was determined the nominal robot geometry and subtracted from the actual length of the bar to obtain the error in the bar length. This error was minimized using the least squares method. The identification problem of minimizing the error in the bar length was linearized. A new method of calculating the Jacobian matrix representing the change in bar length with respect to the robot parameters was developed. This considerably reduced the computational time during identification. 


\section{Chapter 2}

\section{Kinematic Calibration: Why and How?}

\subsection{Why is Kinematic Calibration necessary?}

Significant improvement in accuracy can be achieved by calibrating a robot. Calibration provides a more precise knowledge of the parameters of the actual robot. Some instances where this is useful are listed below:

1. Improvement in accuracy is achieved by modifying the robot positioning software rather than changing the robot design or the control system. This is a major cost saving advantage.

2. By calibrating a robot periodically and studying its results, robot component wear can be monitored.

3. It becomes possible to implement off-line planned and simulated tasks, in real time, due to the high accuracy achieved. 


\subsection{How is Kinematic Calibration achieved?}

Attempts have been made by researchers to classify most of the current approaches to kinematic calibration (referred to as 'calibration' in the rest of the document). Roth et al [2] have classified calibration into three levels: Level I; Level II; Level III. Level I calibration is defined as "joint level" calibration. The goal is to determine the correct relationship between the signal produced by the joint displacement transducer and the actual joint displacement. Level II calibration is defined as the entire robot kinematic calibration. The purpose of calibration at this level is to determine the actual kinematic geometry of the robot as well as correct joint angle relationships. Level III calibration is defined as "non-kinematic calibration". The goal is to calibrate in the presence of the non-geometrical errors like joint flexibility, friction etc.

In general, calibration for all three levels involves four steps: Modelling, Measurement, Identification, and Implementation.

Modelling is the first stage in calibration. It deals with identifying the parameters needed to determine the robot's end-point pose and developing a suitable relationship between these parameters and the end-point pose. The parameters needed to determine the end-point pose may be:

1. Not known exactly. These parameters are also known as 'nominally known' or desired parameters. Calibration provides the exact knowledge of the unknown parameters. These may include link lengths, link offsets etc.

2. Known exactly. These parameters include those that can be measured exactly. These may be encoder readings of the robot joints.

Measurement deals with two aspects: 
1. Measurement of physical data from the actual robot. This may mean devising a measurement system to measure the end-point position and orientation with a very high accuracy. It is important that the accuracy of the measurement system is very high as the calibration procedure assumes that the measurement data obtained is exact.

2. This stage also includes the measurement of parameters that can be measured.

Identification involves mathematical processes using the model developed and the data collected to identify the desired parameters. The measured physical data is used as a 'yard stick' to determine the desired parameters. These parameters are now referred to as 'corrected parameters'.

The implementation phase deals with issues relating to using the corrected parameters in the robot model.

Shamma et al [3] classified calibration as forward and inverse calibration (figure 2). Forward calibration is the process of using encoder readings as input to a model that will yield the actual end point pose. Inverse calibration is the reverse process of calculating the joint position from the desired robot pose. The first three stages (Modelling, Measurement and Identification) can be considered forward calibration, while the Implementation stage can be considered as inverse calibration (figure 2).This thesis deals with only forward calibration of the RoboTwin. The algorithm developed uses the nominal parameters to give the corrected values of the parameters.

\subsubsection{Modelling}

A very suitable choice for developing a robot model is by the use of the DH convention [4]. It was initially developed for representing machine axes. It was later expanded by Paul [5] for application to robotics. The DH convention represents a serial set of linkages by allocating frames 

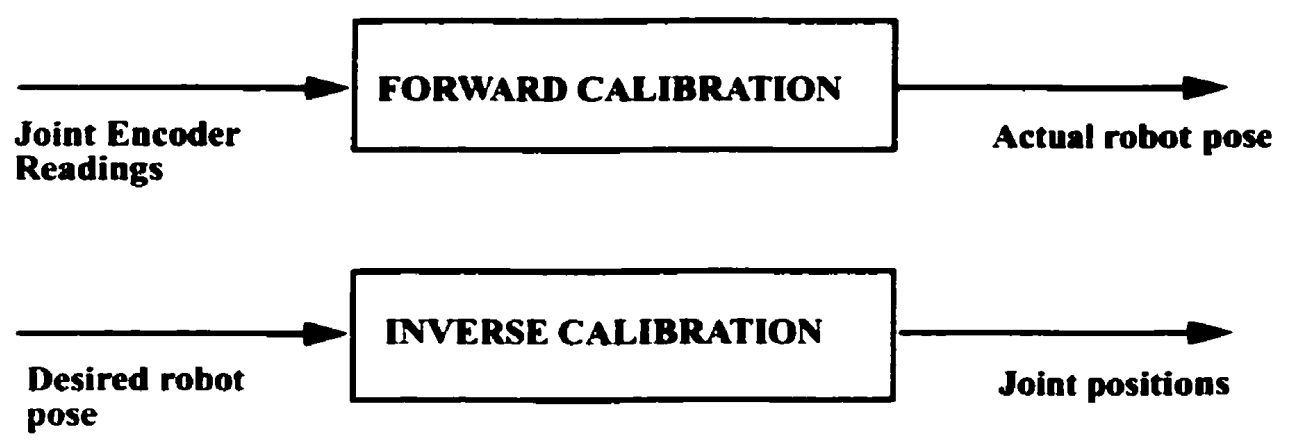

\section{Figure 2. Forward and Inverse Calibration}

to every link and representing the link transformation by means of four parameters. A number of papers have used the DH model for calibration [6].

Unfortunately, a DH model, when used for calibration leads to a singularity [7]. This happens when two consecutive joints are parallel or nearly parallel. The singularity arises due to the fact that a small variation between the actual and nominal model leads to very large variations in the DH parameters. This leads to problems relating to numerical stability while implementing the identification. In some cases, the problem become very highly ill-conditioned and thus unsolvable.

To solve this problem of singularity, many researchers have used different approaches. Ibarra and Perreira [8] attempted to circumvent this problem by assuming that parallel axes are indeed parallel, and slightly misaligned axes can never be calibrated. Hayati [9] modified the D-H parameter representation by replacing one D-H distance parameter with an angle parameter. A modification to the frame allocation convention was also suggested. This modification, popularly known as the Hayati modification, has proved effective only for parallel or near parallel joints and fails when used as a general representation. However, many researchers have used a hybrid 
representation which makes use of Hayati's representation for parallel joints and D-H representation for all other joints $([10],[11])$. Such a representation is popularly known as HayatiDH representation. Many researchers have felt the need for a uniform convention for all robots. Vietschegger and $\mathrm{Wu}$ [12] used a five-parameter representation which included the four DH parameter and the Hayati angle. They claimed the advantages of both D-H and Hayati's representation. However, Schroer [7] proved that the manifold of singularities of Vietschegger convention was similar to that of Hayati DH representation. Many authors have abandoned the DH model altogether to use a six-parameter convention (three parameters for origin displacement and three parameters for relative coordinate system orientation) $([13],[14])$. One model developed by Sheth and Uicker [15] used by Ziegert et al [16] and Goswami et al [17], decomposes the transformation from one link to another into two transformation. The first transformation is a function of joint variable and describes motion of the joint. The second transformation consists of six independent parameters which characterize a particular link. Thus. a complete transformation from one frame to another consists of eight parameters.

The question about the choice of kinematic model led to an attempt by researchers to address a very important question: What are the requirements for a model suitable to be used for calibration? Everett et al [18] identified three desirable properties for a kinematic model to be useful for calibration.

1. Completeness: a model should contain a sufficient number of parameters to completely represent the motion of the robot. Later, Everett and Wong [19] have shown that the maximum number of necessary independent parameters is:

$$
4 \times N-2 \times S+6
$$

where $N$ represents the total number of robot joints and $S$ represents the number of prismatic joints. 
2. Proportionality or Model-Continuity: a small change in the robot geometry should be reflected by a small change in the model parameters. As mentioned before, this is not the case for a DH model used for parallel or near-parallel joint axes. A model lacking continuity gives rise to numerical stability problems.

3. Equivalence: between two acceptable models, there must exist a uniform functional relationship.

Not satisfied with the results of calibration by considering errors due to geometrical factors only, many researchers have tried to incorporate errors due to non-geometrical errors while calibrating the robot $([1],[10])$. Renders [1] has developed a global error model by modeling errors due all possible non-geometrical factors. Results show that consideration of nongeometrical factors is necessary to reduce the error in position below the level of 0.5 to $1 \mathrm{~mm}$. Zeigert [20] recommends that non-geometrical errors should be included in the model only if the desired level of accuracy is not possible otherwise.

\subsubsection{Measurement}

The nature of physical data to be measured for the actual robot depends upon the model developed and the identification method used. The data may include measurement of the complete end-point pose (position and orientation); partial end-point pose (only position); or some aspect of the end-point pose. However, the measurement systems used can be classified based on the measurement technique, independent of the nature of measurement data.

1. Non-contact measurement techniques: These techniques do not involve any contact with the robot structure while performing measurements. Non-contact measurement techniques involve the use of measuring instruments like theodolites, laser interferometers, ultrasonic or sonic devices, infra-red LEDs and cameras. 
A theodolite is simply a telescope that has been instrumented so that the line of sight is very precisely known. It has been used in many measurement techniques in many different ways. Whitney et al [21] have made use of a single theodolite for calibrating a PUMA 560. A target point on the end-point was established and the theodolite was used to determine the line in space on which the target was located. A single theodolite is not capable of providing the distance of a target point from the theodolite. Researchers have therefore made use of triangulation techniques to determine the distance.

Triangulation is based on the determination of the line of sight to a target from several points. The interaction of these lines is used to locate the point. Judd et al [10] calibrated an Automatix AID-900 robot using triangulation with the help of two theodolites. Chen and Chao [14] used triangulation using three theodolites to calibrate a PUMA 560.

Theodolites provide a very high accuracy and allow measurements to be made over a big workspace. However, this technique can be used only statically and not during motion. Furthermore, it is time-consuming.

A laser interferometer uses light interference principles to precisely measure the linear displacement or velocity of the body. Lau, Hocken and Haight [22] have made use of a measurement system consisting of a laser interferometer with a steerable beam to measure the distance of a target point on the robot.

Ultrasonic or sonic devices are based on 'time of flight' principle. An ultrasonic or sonic pulse is emitted by an emitter (source) and received by a receiver. The time of flight for the pulse is measured and the distance is calculated. Stone et al ([23], [24]) have used an acoustic emitter located on the target point with microphones located at 
points throughout the workspace. A major disadvantage of these systems is the sensitivity of the speed of sound to air temperature and humidity. Therefore, the calibration has to be performed many times for accurate results.

Infra-red LEDs have been used in a 3D measurement system by Hollerbach [25] and An et al [26]. An IR LED is mounted on the end-effector and a stereoscopic analysis is carried out by two cameras to collect data to locate the end-point of the robot.

2. Contact measurement techniques: These techniques collect end-point pose information by making contact with the robot structure while performing measurements. They make use of devices such as coordinate measuring machines, dial indicators, fixtures, linear variable differential transformers (LVDTs) and probes to make measurements.

Coordinate measurement machines (CMM) are precise 3-dof mechanisms with three orthogonal prismatic axes. At the tip of its Z-axis, is a touch probe that is used to locate the position of the body with respect to the CMM. The use of CMMs is very appealing because of its ease of use. Unfortunately, most CMMs are too expensive to justify the use for calibration alone.Vietschegger and Wu [27] have used a CMM to construct a precise fixture plate having a set of precisely positioned holes. The end effector had a pointing device. The end-point was made to move to these previously located points and joint encoder data noted. Borm and Menq [28] have calibrated a RM501 robot by using a CMM and a simple cylinder as an end-effector. The CMM was used to locate four points on the cylinder face and four points around the circumference of the cylinder. This partial pose information was then used for calibration.

Dial indicators have been used as short range sensor for calibration [29]. These are available in two types: mechanical and digital. Mechanical indicators have to be manually read and are unsuitable for automated measurement. Geo-ry Tang et al [29] 
have use a electronic micrometer attached to the end-point of the robot. During data collection the robot is moved on a flat surface and the distance readings are noted by the micrometer.

3. Special measurement techniques: The issue of the contact with the robot becomes completely irrelevant in these techniques. These techniques are also called as passive measurement schemes. Hollerbach et al ([30],[31]) developed and simulated a passive measurement scheme known as 'closed loop kinematic calibration'. They argued that if a manipulator is formed into a mobile closed kinematic chain, then its joint angle readings alone were enough to identify the kinematic parameters. A mobile closed chain could be formed if it was redundant with respect to its end point constraint. This approach found an immediate application in calibrating robots performing tasks like opening a door as manipulators naturally form mobile closed kinematic chains while performing these tasks [32]. The authors used this approach in calibrating a manipulator and camera system [33], RSI 6-dof hand [34] and the Utah-MIT hand [35].

Driels [36] used a passive measurement technique to calibrate a PUMA 500 robot. A ball-bar mechanism was used as an extra joint to form a mobile closed chain robot. The ball-bar consisted of a bar having two spherical joints at both ends. This arrangement gave it an additional six dofs. Both joints were connected to plates. One plate was fixed to a point on the ground (work table) and the other was attached to the end-effector. The robot motors were given no current and the robot was manually moved into 45 poses to collect the joint encoder data. The data collected was used for identification of the unknown parameters. 
Goswami et al [17] also used a similar technique using a telescopic ball-bar system. The ball-bar had a LVDT to measure the distance of the robot end-point from a fixed location. The ball-bar had a magnetic chuck permanently mounted on one end and a removable sphere mounted on the other. An additional magnetic chuck fixed to the ground mated with the steel ball on one end of the ball-bar to form a spherical joint. The chuck end of the ball-bar mated with the steel ball on the moving end-point of the robot. The robot was then moved in the workspace to collect joint encoder and LVDT readings.

\subsubsection{Identification}

Consider two robots $\mathbf{A}$ and $\mathrm{B}$. Robot $\mathbf{A}$ is the robot as defined by the designer. It is represented mathematically by means of a model and designer specified parameters (also referred to as nominal parameters). Robot B is the actual robot structure. Robot B parameters differ from those of robot $\mathbf{A}$ due to reasons discussed previously in this chapter. The identification stage uses the model A and measurements made on the structure of B to determine a parameters for robot B (also referred to actual parameters).

Mathematically, the identification phase can be explained as follows:

If $\vec{q}$ represents the set of parameters representing a robot, then $\vec{q}_{A}$ and $\vec{q}_{B}$ represent the parameters for robot $A$ and robot $B$, respectively. The robot pose $\vec{x}$ can be written as,

$$
\vec{x}=\left(\begin{array}{l}
\vec{p} \\
\vec{\rho}
\end{array}\right)=f(\vec{q}, \vec{\Theta})
$$

where, $\vec{p}$ is the position vector, $\vec{\rho}$ is the orientation of the end-effector frame and $\vec{\theta}$ is the joint variable vector. The pose for robots $A$ and $B$ can be written as,

$$
\overrightarrow{\boldsymbol{x}}_{A}=\left(\begin{array}{l}
\vec{p}_{A} \\
\overrightarrow{\boldsymbol{\rho}}_{A}
\end{array}\right)=f\left(\vec{q}_{A}, \overrightarrow{\mathbf{\Theta}}\right)
$$




$$
\overrightarrow{\boldsymbol{x}}_{B}=\left(\begin{array}{l}
\vec{p}_{B} \\
\vec{\rho}_{B}
\end{array}\right)=f\left(\vec{q}_{B}, \vec{\Theta}\right)
$$

where, $\vec{p}_{A}, \vec{\rho}_{A}$ and $\vec{p}_{B}, \vec{\rho}_{B}$ are the position vectors and orientations of the end-effectors, respectively. The identification phase uses the model of the robot and measurements made on the pose $\vec{x}_{B}$ and the joint variable readings $\vec{\Theta}$ to determine the parameters of robot $\mathrm{B}, \vec{q}_{B}$.

Two approaches have been used to determine the parameters of robot B (or actual parameters): geometric approach and parametric approach. The geometric approach identifies the joint axes in space of the actual robot. The actual parameters are extracted from the actual robot geometry. The parametric approach on the other hand determines the error between the end-point pose of the actual robot (robot B) and the end-point pose of the nominal robot (robot A). This error is then used to determine the parameters of the actual robot.

One geometric approach is to move each joint of the robot to a goal point. Simultaneously, the motion of end-point is traced. For a perfect revolute joint, the end-point will move on a circular trajectory. The axis of rotation can be estimated from the trajectory. Stone et al ([23],[24],[37]) have used this approach by use of acoustic transducers mounted on each link. Sklar [38] has used a similar approach and called it Circle Point Analysis (CPA). However, only axes about which real motions occurs and can be located in space, and can be identified by this approach.

The parametric approach makes use of the Taylor's series approximation for the pose data measured. The assumption here is that the values of nominal parameters are close to the actual parameters to be determined.

$$
\begin{gathered}
\vec{x}_{B}=\vec{x}_{A}+\frac{\partial \vec{x}_{A}}{\partial \vec{q}_{A}} \cdot \Delta \vec{q}_{A}+\frac{\partial \vec{x}_{A}}{\partial \vec{q}_{A}^{2}} \cdot \Delta \vec{q}_{A}^{2}+\ldots \\
\vec{x}_{B}=\vec{x}_{A}+J \cdot \Delta \vec{q}_{A}+H \cdot \Delta \vec{q}_{A}^{2}+\ldots
\end{gathered}
$$




$$
\begin{gathered}
J=\frac{\partial \vec{x}_{A}}{\partial \vec{q}_{A}} \\
H=\frac{\partial^{2} \vec{x}_{A}}{\partial \vec{q}_{A}^{2}}
\end{gathered}
$$

where, $\vec{x}_{B}$ is the actual pose (measured), $\vec{x}_{A}$ is the nominal pose calculated by equation (3), $\vec{q}_{A}$ is the nominal parameter vector, $\Delta \vec{q}_{A}$ is the error in the nominal parameters, $J$ is the Jacobian matrix and $H$ is the Hessian matrix. The error in the parameters is determined by solving equation (5). Two approaches have been used to solve the equation: linear and non-linear.

The linear solution approach locally linearizes equation (5) by ignoring higher order terms of $\Delta \vec{p}_{A}$. The equation (6) can be written as,

$$
\begin{gathered}
\Delta \vec{x}=\vec{x}_{B}-\vec{x}_{A}=J \cdot \Delta \vec{q}_{A} \\
\Delta \vec{x}=J \cdot \Delta \vec{q}_{A}
\end{gathered}
$$

where, is $\Delta \vec{x}$ the error in the end-point pose.

Equation (10) can be solved by linear least squares method, which minimizes the square of the error in pose, $\Delta \vec{x}$, to give,

$$
\Delta \vec{q}_{A}=\left(J^{T} \cdot J\right)^{-1} \cdot J^{T} \cdot \Delta \vec{x}
$$

where $\left({ }^{*}\right)^{T}$ represents the transpose of the vector or matrix.

This linearized solution provides a local solution for $\Delta \vec{q}_{A}$. Many researchers have used this solution iteratively to obtain the corrected parameters. The value of $\Delta \vec{q}_{A}$ can be used to obtain an updated value for $\vec{q}_{A}$.

$$
\vec{q}_{A}^{\text {new }}=\vec{q}_{A}^{\text {old }}+\Delta \vec{q}_{A}
$$


The updated value of the parameters can used in the identification algorithm once again. New estimates of the error in parameters can be obtained till the error is zero or less than a specified tolerance.

Some researchers have chosen not to linearize equation (6) and have used non-linear methods to determine the error in the parameters. Driels et al ([36],[39],[40]) have made use of a standard IMSL library to solve the non-linear least squares problem. Ananthan et al [41] have used a modified Newton's method to achieve faster convergence.

\subsubsection{Implementation}

The Implementation phase deals with the usage of the actual robot parameters (determined by the first three phases) in the robot control software. This phase is also referred to 'error compensation'.

The inverse kinematic solution in the robot's controller is based on a simplified robot model (for example, with joints axes assumed to either perfectly parallel or normal to each other). This allows calculation of an efficient closed-form solution of the inverse kinematics. In an actual robot, the joint axes will be slightly misaligned. For such a robot, a closed form solution may not be practical or possible to find [42]. 


\section{Chapter 3}

\section{An Algorithm for RoboTwin Calibration}

The RoboTwin is a reconfigurable, modular and expandable robot. It is a patented and commercially viable robot developed at Robotics and Automation Lab (RAL), Department of Mechanical Engineering, University of Toronto. Development of a calibration system for this robot is very important for achieving better accuracy. Calibrating such a system possesses a new challenge due to its unique characteristic of reconfigurability.

\subsection{The RoboTwin: A modular, re-configurable and expandable robot}

The uniqueness of the robot arises due to its re-configurability, modularity and expandablity. These three properties are reflected in its mechanical structure and its control architecture. Both the mechanical structure and the control architecture are discussed in the following sections.

\subsubsection{Mechanical Structure}

The RoboTwin can be best described as a 'lego set' consisting of eight revolute joints, connecting linkages and grippers. The joints can be attached to linkages by means of set-screws 
and the gripper can be attached to its last link. The robot can be configured in one of many configurations (figure 3) thus making the robot structure re-configurable. The process of disassembling a configuration and assembling a new configuration takes less than ten minutes.

Two types of joints are available for the RoboTwin: Type I and Type II. These are shown schematically in figure 4. In Type I joints, the axis of rotation is perpendicular to at least one of the contiguous links. In Type II joints the axis of the robot is parallel to the link. A combination of both types of joints makes it possible to have a wider range of configurations.

Of the eight joints, two are reconfigurable. These joints are designed so they can behave either as Type I or Type II. However, these joints work out to be bulky and heavy and are thus used near the base and not as proximal joints.

The robot structure is expandable as additional joints and grippers can be manufactured and used.

The RoboTwin also exhibits modularity. Each robot joint is a separate module consisting of an electric motor controlled by one D/A channel. Each joint consists of a dc motor, a harmonic drive, a torque sensor and an optical encoder. A schematic diagram of the robot joint is shown in figure 5.[44]

Figure 6 [44] shows the schematic diagram of the reconfigurable joint. The distal link can be connected to end $\mathrm{A}$ to use it as a Type I joint or to end $\mathrm{C}$ to use it as a Type II joint. The end $\mathrm{A}$ is detachable.

In its base-line configuration, the robot is configured as two arms with four joints each (This is also the reason for its name). The base line configuration is shown in figure 7. Configured as a dual-arm robot it can be used for coordinated motion, grasping and rolling applications [43]. 

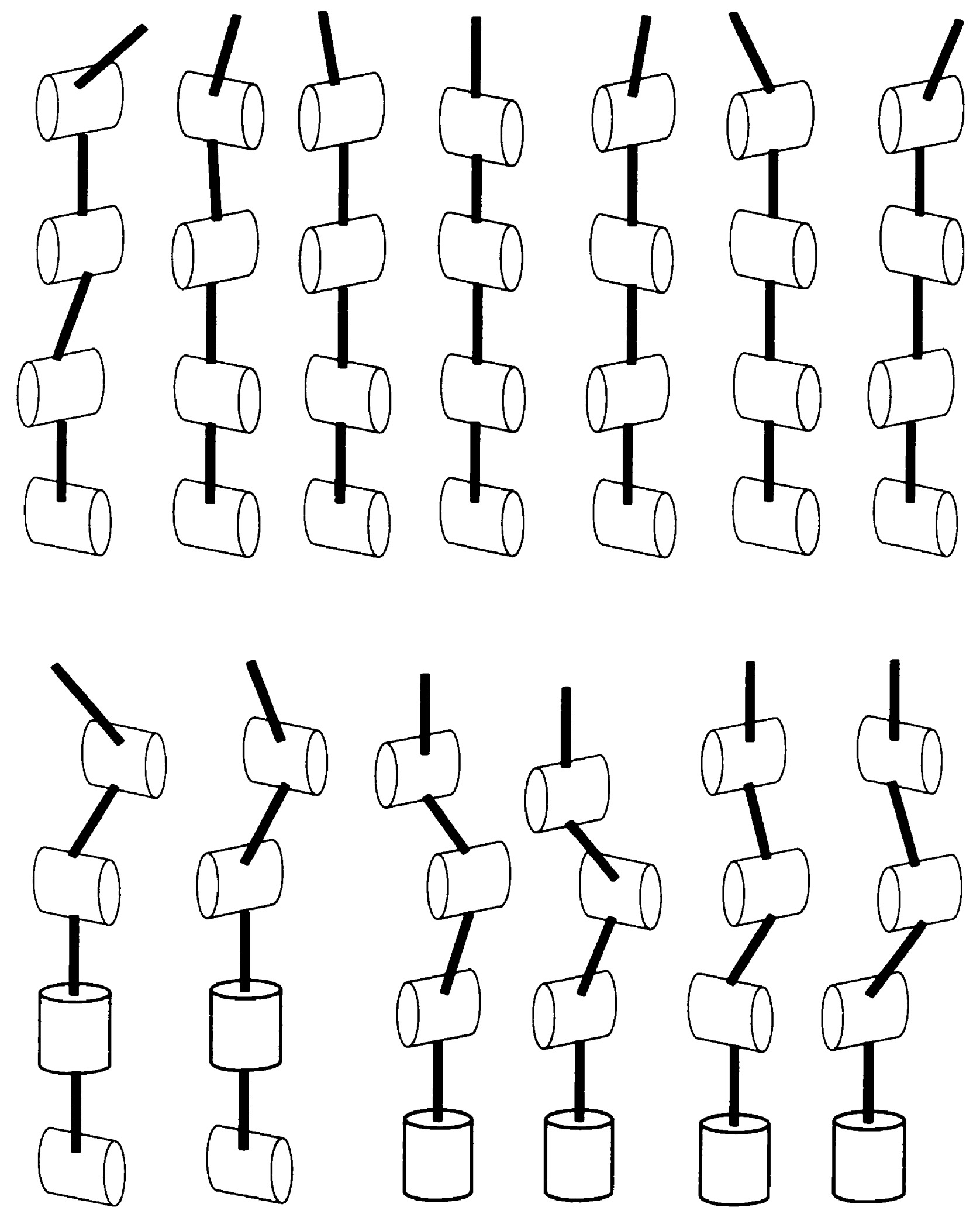

Figure 3. Possible Configurations of RoboTwin 


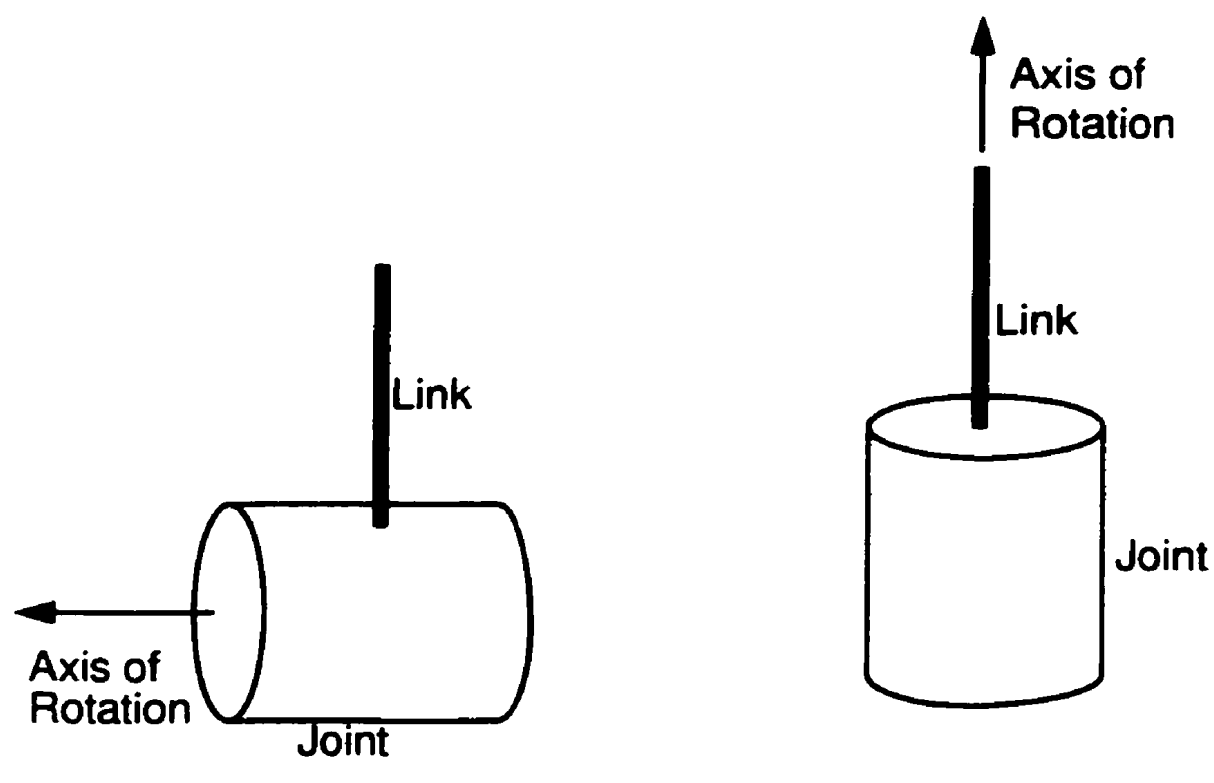

Type I Joints

Type II Joints

Figure 4. Types of RoboTwin Joints

\subsubsection{Control Architecture}

The real time controller of the RoboTwin has also been designed to be modular and expandable. The control system consists of one computer node running two arms simultaneously. Additional arms can be controlled by using additional identical nodes making the system expandable.

The node (figure 8) is built around a 50 MHz EISA bus-based IBM-PC compatible host computer. A RISC co-processor board (ATM-29050) is attached to the PC bus. The RISC board has a powerful in floating point unit and its own memory. The control system hardware includes three I/O boards attached to its host bus: (i) ADC boards (ii) DAC boards (iii) Digital I/O boards. 


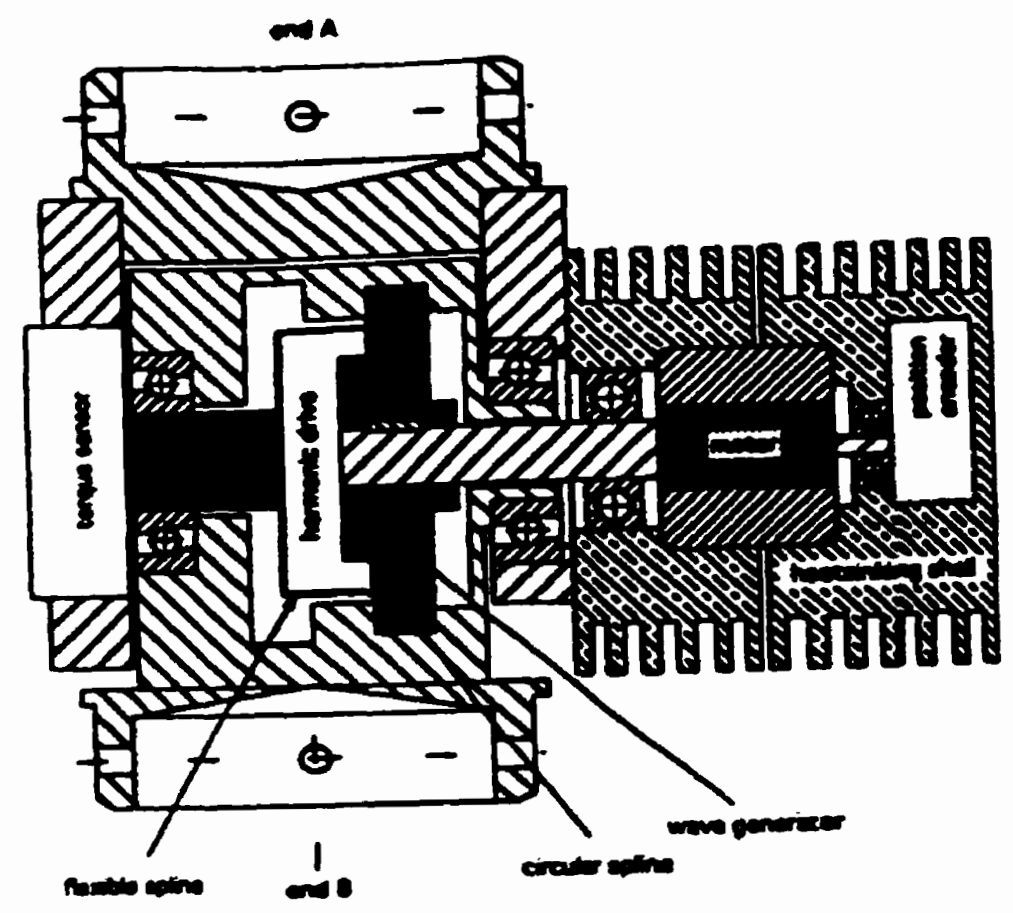

Figure 5. Schematic Diagram of Robot Joints

The L/O boards are connected to the low level control system that interfaces to the optical encoders, signal conditioning circuits and power amplifiers [45].

\subsection{Design Criterion}

The primary aspect of the calibration system designed was to accommodate reconfigurability and expandability.

Every time the robot is re-configured to perform a different task, calibration becomes imperative. Disassembling and re-assembling the robot lead to mounting errors which reflect a variation between the nominal and actual robot geometry. Moreover, every operation of re- 


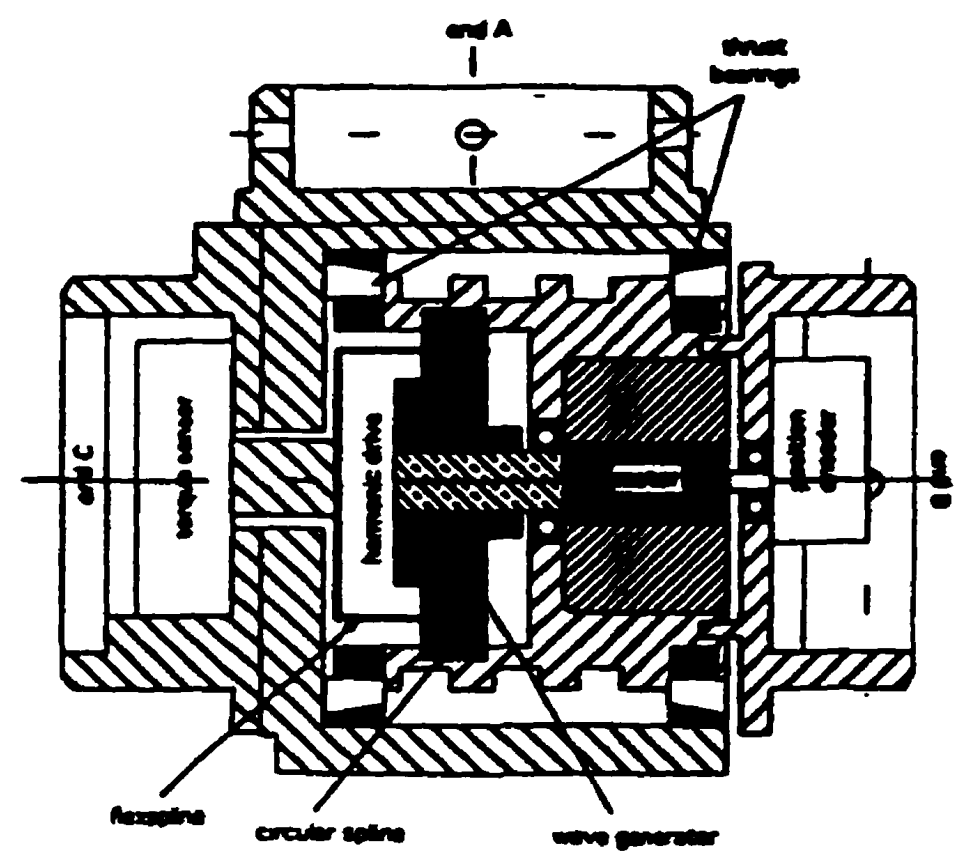

Figure 6. Schematic Diagram of Re-configurable Joints

configuring the robot leads to a unique geometry of the real robot. Even disassembling and reassembling the same configuration may lead to a different real robot. The calibration system should be independent of the chosen configuration in its modelling method, measurement strategy and the implemented software.

In its current form, the RoboTwin consists of eight joints and two grippers. However, as mentioned in section 3.1 , it is capable of being expanded to include more joints and linkages. It is capable of using a 3-dof wrist due to the system's expandability (A 3-dof wrist is currently being manufactured). The calibration system should be able to successfully incorporate more joints and linkages or a wrist, if dictated by need, with minimum effort.

As mentioned earlier, the RoboTwin will have to be calibrated every time it is re-configured. This could be quite often. Due to its perceived frequent use, the calibration system should be easy 


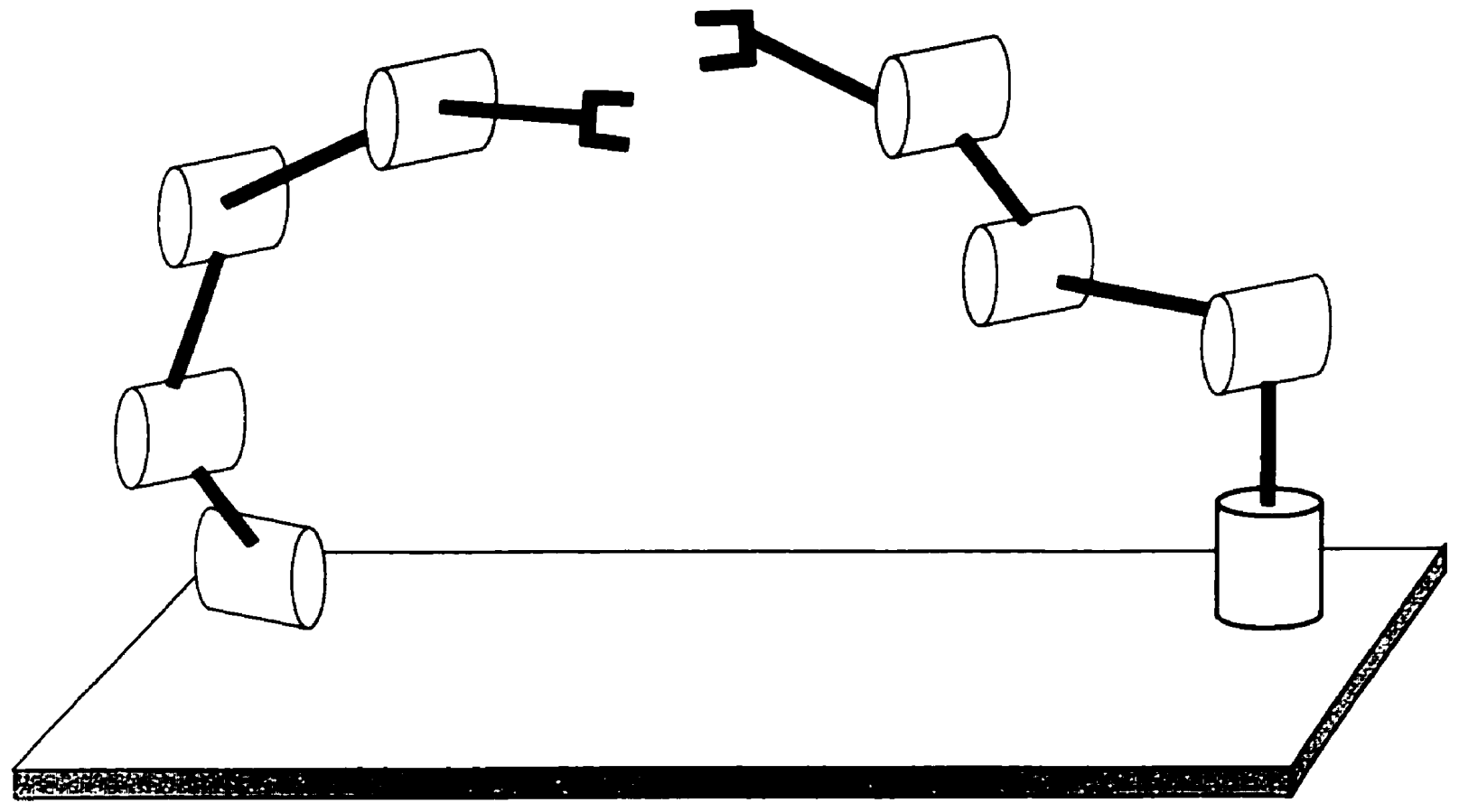

Figure 7. Base-line Configuration of RoboTwin

to use. The ease of use should be reflected in a user-friendly calibration interface. The modeling method used should be widely accepted. This would enable users to use calibration results without many changes to their control algorithm. The measurement strategy should not be cumbersome and tiring. The calibration procedure should be implemented without moving the robot from its workplace.

Finally, the cost of the calibration system should be proportionate with the cost of the RoboTwin. 


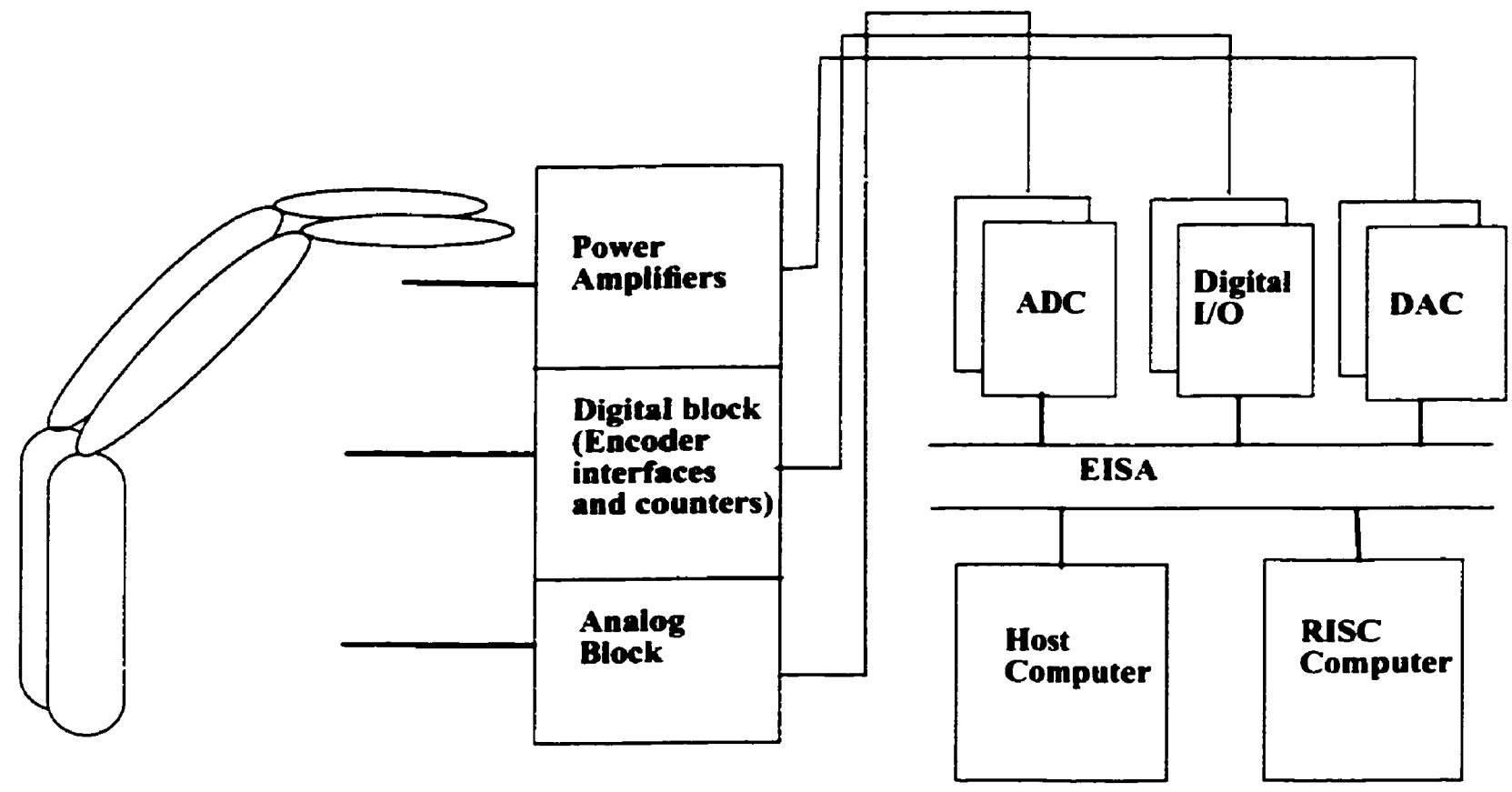

Figure 8. Control Architecture

To summarize, the following design criterion were established for developing a calibration system for the RoboTwin.

1. The calibration system should be independent of the chosen configuration;

2. The calibration system should be expandable to incorporate additional joints or wrists;

3. The calibration system should be easy to use. This should be reflected in:

- an easy to use calibration interface;

- use of a widely accepted modelling method;

- a measurement strategy that is not tiring and cumbersome; 
- implementation of calibration at its work place;

4. The calibration system should not be expensive.

\subsection{An Algorithm for Calibration}

A calibration system was designed using the criterion established in section 3.2 . In this section, the algorithm for calibration is presented. The process consists of the Modelling, Measurement and Identification phases. A summary of the design is presented at the end of this section.

\subsubsection{Modelling}

Due to its wide use by researchers in robotics, the Denavit-Hartenberg convention (DH convention) would be a good choice as a modelling methodology for developing a calibration system. Many control algorithms used for controlling the RoboTwin are implemented using D-H parameters. Calibration would provide the correct values of the D-H parameters and enhance the robot performance. The calibration results could be used in conjunction with any control algorithm based on the D-H convention.

As explained in section 2.2.1, the DH model is not suitable for calibration as it leads to a singularity when two consecutive joints are parallel. Heuristically, a singularity is observed when a small change in the joint variables generates a large change in the position and orientation of the end-effector [7]. Mathematically, a singularity is seen when the Jacobian matrix becomes rank deficient. Such a singularity is observed in the DH model when used for nominally parallel joints, making the model discontinuous. Many researchers have worked with alternative models (based on more than four parameters) to represent a homogeneous transformation. However, using a 
model that provides results in terms of parameters that are not widely accepted or are relatively unknown, would conflict with our design criterion of making the algorithm easy to implement. Hayati modified the D-H convention [9] for the parallel joint case and this has been a popular choice amongst researchers.

\subsubsection{The DH Model}

Consider an open-loop kinematic chain consisting of $N$ linkages and $(N+I)$ joints. The D-H convention labels the linkages from 0 to $n$ where link 0 represents the base. The joints are labelled $I$ to $(N+1)$. Joint $i$ connects links $(i-1)$ and $i$ where $i=1 \ldots(N+1)$.

The manipulator (open loop kinematic chain) can be modelled by assigning coordinate frames and representing the frames relative to each other by means of homogeneous transformations. In the D-H convention frames are attached to the joints such that their axes of rotation align with the $\mathrm{Z}$-axis of the coordinate frame. The frames are labelled from $O$ to $N$ corresponding to the linkages. The allocation of frames are as in the figure 9.

The frames are assigned to the linkages based on the following rules.

Base frame $\left(X_{0} Y_{0} Z_{0}\right)$ allocation

- Origin, $O_{0}$ located arbitrarily

- $Z$ axis, $Z_{0}$ aligns with the axis of joint 1

- $\mathrm{X}$ axis, $X_{0}$ oriented arbitrarily

- $\mathrm{Y}$ axis, $Y_{0}$ oriented in accordance with the right hand coordinate system

Link $i$ frame allocation $\left(X_{i} Y_{i} Z_{i}\right)$ where $i=1 \ldots(N-1)$ 


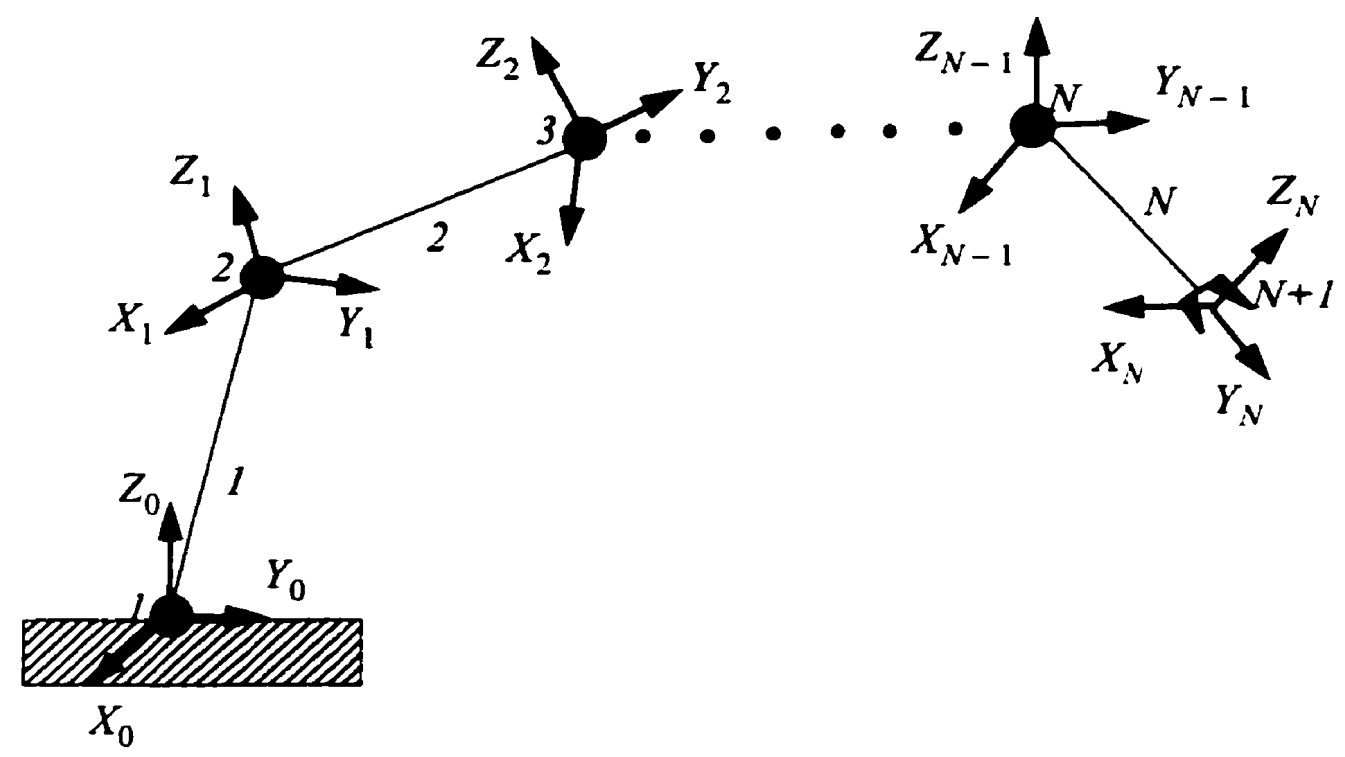

Figure 9. Labelling and Allocation of Frames in an open loop kinematic chain

- Origin, $O_{i}$ located at the intersection of the axis of rotation (joint axis) for joint $(i+1)$ and the common normal between the joint axes for link $i$ and $(i+1)$.

- $Z$ axis, $Z_{i}$ aligns with the axis of joint $(i+l)$

- $\mathrm{X}$ axis, $X_{i}$ aligns with the common normal between the joint axes for link $i$ and $(i+1)$

- $\mathrm{Y}$ axis, $Y_{i}$ oriented in accordance with the right hand coordinate system

End effector frame allocation $\left(X_{N} Y_{N} Z_{N}\right)$

- Origin, $O_{N}$ arbitrarily on the end-effector

- $Z$ axis, $Z_{N}$ aligns with the $Z$ axis of the previous frame, $Z_{N-1}$

- $\mathrm{X}$ axis, $X_{N}$ aligns with the common normal between $Z_{N-1}$ and $Z_{N}$

- $\mathrm{Y}$ axis, $Y_{N}$ oriented in accordance with the right hand coordinates system 
The location and orientation of frame $i$ with respect to frame $(i-l)$ is determined by means of a homogeneous transformation, $H_{i-1}^{i}$. The location and orientation of the end effector frame with respect to the base frame, $H_{0}^{N}$ can then be determined by $H_{i-1}^{i}$ as shown in the following equation.

$$
H_{0}^{N}=H_{0}^{1} \cdot H_{1}^{2} \cdot \ldots \cdot H_{i-1}^{i} \cdot \ldots \cdot H_{N-1}^{N}
$$

Four parameters are used to represent the transformation from frame (i-1) to $i$ in the D-H convention. These are the link length $\left(l_{i}\right)$, link offset $\left(r_{i}\right)$, twist angle $\left(\alpha_{i}\right)$ and joint angle $\left(\Theta_{i}\right)$. These four parameters and the relationship between two consecutive frames $(i-1)$ and $i$ is shown in the figure 10. Link length represents the distance along the common normal between the two consecutive $\mathrm{Z}$-axes. The link offset represents the distance between the origin of frame $(i-l)$ and the point of intersection of the common normal and $Z$-axis of frame (i-1). The twist angle represents the angle between the $Z$-axes of the two consecutive frames measured from $Z_{i-1}$ to $Z_{i}$. The joint angle represents the angle between the $\mathrm{X}$-axes of the two consecutive frames measured from $X_{i-1}$ to $X_{i}$.

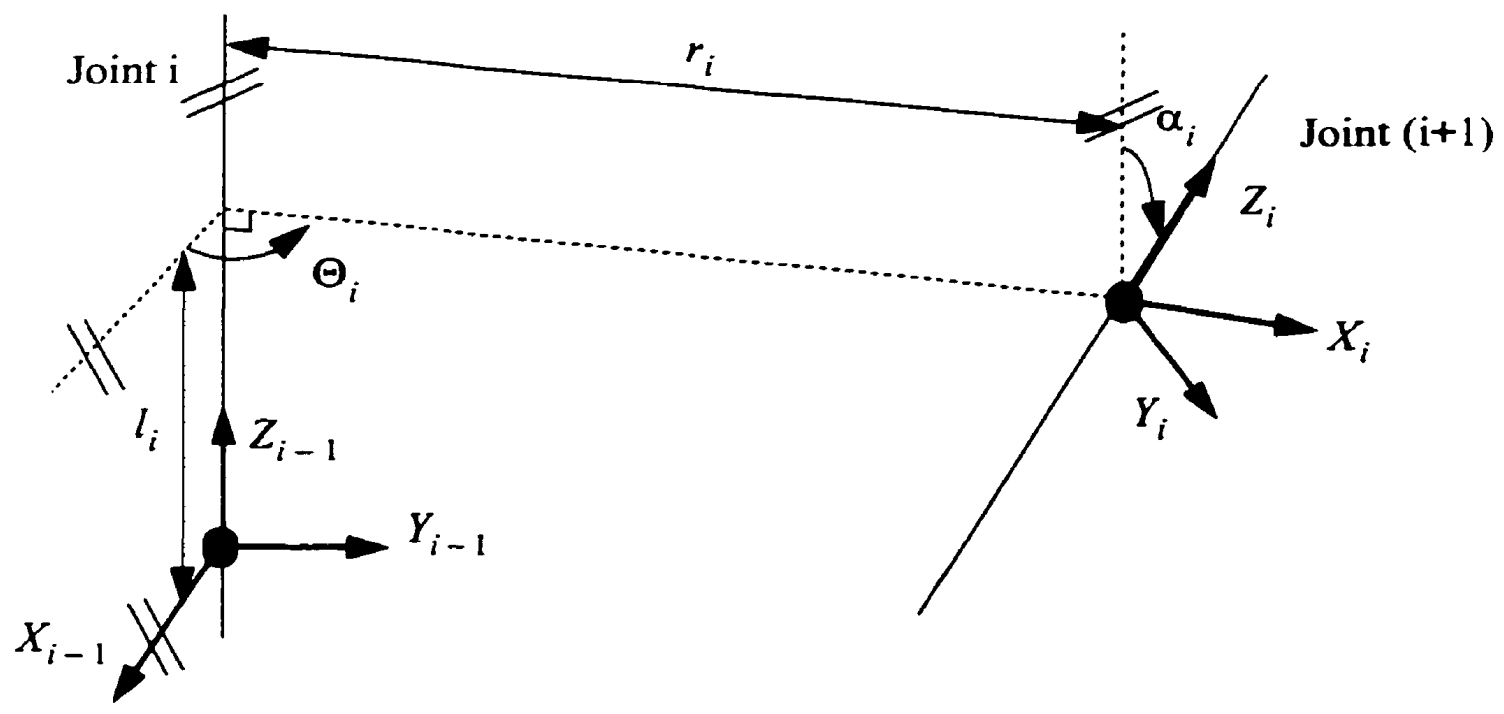

Figure 10. Representation of D-H parameters 
The homogeneous transformation $H_{i-1}^{i}$ is obtained by means of two rotation and two translation transformation.

- Rotation about $Z_{i-1}$ by $\Theta_{i}, R\left(Z_{i-1}, \Theta_{i}\right)$

- Translation along $Z_{i-1}$ by $r_{i}, T\left(Z_{i-1}, r_{i}\right)$

- Translation along $X_{i}$ by $l_{i}, T\left(X_{i}, l_{i}\right)$

- Rotation about $X_{i}$ by $\alpha_{i}, R\left(X_{i}, \alpha_{i}\right)$

$R($.$) represents a rotation transformation and T($.$) represents a translation transformation$

$$
\begin{aligned}
& H_{i-1}^{i}=R\left(Z_{i-1}, \Theta_{i}\right) \cdot T\left(Z_{i-1}, r_{i}\right) \cdot T\left(X_{i}, l_{i}\right) \cdot R\left(X_{i}, \alpha_{i}\right)
\end{aligned}
$$

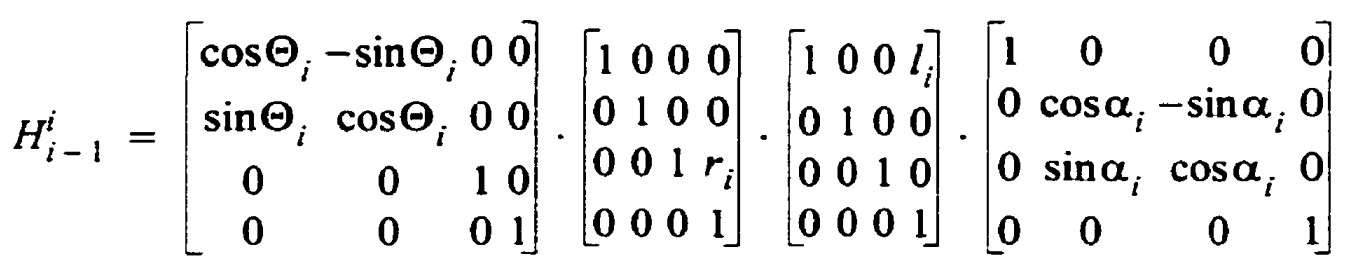

$$
\begin{aligned}
& H_{i-1}^{i}=\left[\begin{array}{cccc}
\cos \Theta_{i} & -\sin \Theta_{i} \cdot \cos \alpha_{i} & \sin \Theta_{i} \cdot \sin \alpha_{i} & l_{i} \cdot \cos \Theta_{i} \\
\sin \Theta_{i} & \cos \Theta_{i} \cdot \cos \alpha_{i} & -\cos \Theta_{i} \cdot \sin \alpha_{i} & l_{i} \cdot \sin \Theta_{i} \\
0 & \sin \alpha_{i} & \cos \alpha_{i} & r_{i} \\
0 & 0 & 0 & 1
\end{array}\right]
\end{aligned}
$$

\subsubsection{Hayati-DH Model for Near-Parallel Rotary Joints}

Hayati introduced a new set of rules to determine the link parameters for the special case of two consecutive near-parallel joints [9]. The new link coordinate frame for frame $i$ is obtained by following the four step outlined below (refer to figure 11).

1. Pass a plane perpendicular to the $Z_{i-1}$ axis. The intersection of this plane with the joint axis $(i+1)$ is $O_{i}$ (Origin of frame i). 


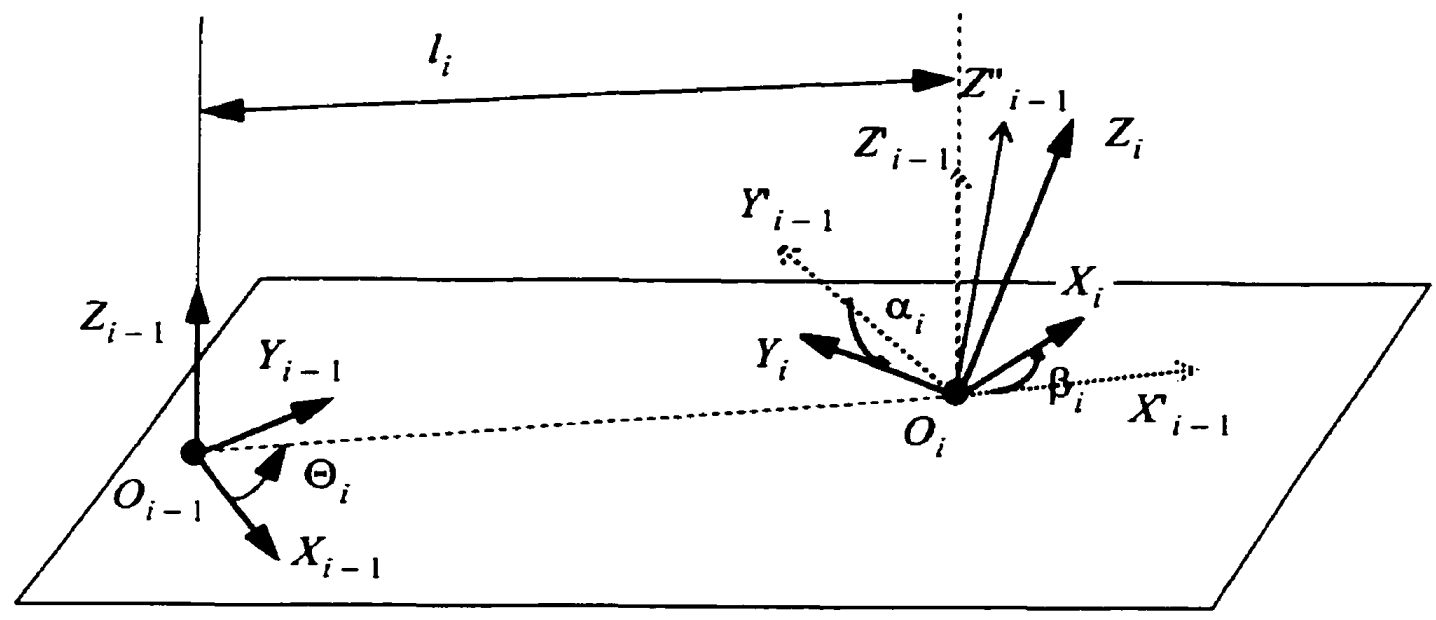

Figure 11. Representation of Hayati parameters

2. Rotate the frame (i-1) about $Z_{i-1}$ to align $X_{i-1}$ with the line connecting the points $O_{i-1}$ and $O_{i}$.

3. Translate the origin of the last frame to $O_{i}$ and obtain frame $X_{i-1} Y_{i-1} Z_{i-1}$

4. Perform a rotation about $X_{i-1}$ to obtain the frame $X^{\prime \prime}{ }_{i-1} Y^{\prime \prime}{ }_{i-1} Z^{\prime \prime}{ }_{i-1}$ so that $Y_{i-1}$ aligns with $Y_{i}$.

5. Perform a rotation about $Y^{\prime \prime}{ }_{i-1}$ to obtain the frame $X_{i} Y_{i} Z_{i}$ so that $X_{i-1}$ aligns with $X_{i}$.

The Hayati representation makes use of four parameters for transforming the frame (i-1) to frame $i$ which represents a linkage connected by two nominally parallel joints. These are the joint angle $\left(\Theta_{i}\right)$, link length $\left(l_{i}\right)$, twist angle $\left(\alpha_{i}\right)$ and the Hayati angle $\left(\beta_{i}\right)$. The joint angle $\left(\Theta_{i}\right)$ represents the angle between $X_{i-1}$ and $X_{i-1}$. The link length $\left(l_{i}\right)$ represents the distance 
between the origins of the frame (i-l) and $i$. The twist angle $\left(\alpha_{i}\right)$ represents the angle between $Y_{i-1}$ and $Y_{i}$. The Hayati angle $\left(\beta_{i}\right)$ represents the angle between $X_{i-1}$ and $X_{i}$.

The homogeneous transformation $H_{i-1}^{i}$ can be obtained by means of three rotation and one translation transformation.

- Rotation about $Z_{i-1}$ by $\Theta_{i}, R\left(Z_{i-1}, \Theta_{i}\right)$

- Translation along $X_{i-1}$ by $l_{i}, T\left(X_{i-1}, l_{i}\right)$

- Rotation about $X_{i-1}$ by $\alpha_{i}, R\left(X_{i-1}, \alpha_{i}\right)$

- Rotation about $Y^{\prime \prime}{ }_{i-1}$ by $\beta_{i}, R\left(Y^{\prime \prime}{ }_{i-1}, \beta_{i}\right)$

$R$ (.) represents a rotation transformation and $T($.$) represents a translation transformation$

$$
\begin{gathered}
H_{i-1}^{i}=R\left(Z_{i-1}, \theta_{i}\right) \cdot T\left(X_{i-1}, l_{i}\right) \cdot R\left(X_{i-1}, \alpha_{i}\right) \cdot R\left(Y_{i-1}^{\prime \prime}, \beta_{i}\right) \\
H_{i-1}^{i}=\left[\begin{array}{crrr}
\cos \Theta_{i}-\sin \Theta_{i} & 0 & 0 \\
\sin \Theta_{i} & \cos \Theta_{i} & 0 & 0 \\
0 & 0 & 1 & 0 \\
0 & 0 & 0 & 1
\end{array}\right] \cdot\left[\begin{array}{cccc}
1 & 0 & 0 & l_{i} \\
0 & 1 & 0 & 0 \\
0 & 0 & 1 & 0 \\
0 & 0 & 0 & 1
\end{array}\right] \cdot\left[\begin{array}{cccc}
1 & 0 & 0 & 0 \\
0 & \cos \alpha_{i}-\sin \alpha_{i} & 0 \\
0 & \sin \alpha_{i} & \cos \alpha_{i} & 0 \\
0 & 0 & 0 & 1
\end{array}\right] \cdot\left[\begin{array}{cccc}
\cos \beta_{i} & 0 & \sin \beta_{i} & 0 \\
0 & 1 & 0 & 0 \\
\sin \beta_{i} & 0 & \cos \beta_{i} & 0 \\
0 & 0 & 0 & 1
\end{array}\right] \\
H_{i-1}^{i}=\left[\begin{array}{cccc}
-s \alpha_{i} \cdot s \beta_{i} \cdot s \Theta_{i}+c \beta_{i} \cdot c \Theta_{i} & -c \alpha_{i} \cdot s \Theta_{i} & s \alpha_{i} \cdot c \beta_{i} \cdot s \Theta_{i}+s \beta_{i} \cdot c \Theta_{i} & l_{i} \cdot c \Theta_{i} \\
s \alpha_{i} \cdot s \beta_{i} \cdot c \Theta_{i}+c \beta_{i} \cdot s \Theta_{i} & c \alpha_{i} \cdot c \Theta_{i} & -s \alpha_{i} \cdot c \beta_{i} \cdot c \Theta_{i}+s \beta_{i} \cdot s \Theta_{i} & l_{i} \cdot s \Theta_{i} \\
-c \alpha_{i} \cdot s \beta_{i} & s \alpha_{i} & c \alpha_{i} \cdot c \beta_{i} & 0 \\
0 & 0 & 0 & 1
\end{array}\right]
\end{gathered}
$$

In equation (19), $\mathrm{c}$ represents $\cos ($.$) and \mathrm{s}$ represents $\sin ($.

\subsubsection{A Model for RoboTwin}

The homogeneous transformation, $H_{0}^{N}$ can be determined using $H_{i-1}^{i}$ by equation (13) where, 


$$
\begin{gathered}
H_{i-1}^{i}=f\left(\Theta_{i} l_{i}, \alpha_{i}, \beta_{i}\right) \text { for nominally parallel joints } \\
H_{i-1}^{i}=f\left(\Theta_{i}, l_{i}, r_{i}, \alpha_{i}\right) \text { for all other joints }
\end{gathered}
$$

The homogeneous transformation, $H_{0}^{N}$ can be written as,

$$
H_{0}^{N}=H_{0}^{1} \cdot H_{1}^{2} \cdot \ldots \cdot H_{i-1}^{i} \cdot \ldots \cdot H_{N-1}^{N}=f(\grave{l}, \vec{\alpha}, \vec{\Theta}, \vec{u})
$$

where,

$$
\begin{gathered}
i=\left[\begin{array}{llll}
l_{1} & l_{2} & \ldots & l_{N}
\end{array}\right]^{T}, \dot{l} \in \mathfrak{R}^{N \times 1} \\
\vec{\alpha}=\left[\begin{array}{llll}
\alpha_{1} & \alpha_{2} & \ldots & \alpha_{N}
\end{array}\right]^{T}, \vec{\alpha} \in \mathfrak{R}^{N \times 1} \\
\vec{\Theta}=\left[\begin{array}{llll}
\Theta_{1} & \Theta_{2} & \ldots & \Theta_{N}
\end{array}\right]^{T}, \vec{\Theta} \in \mathfrak{R}^{N \times 1} \\
\vec{u}=\left[\begin{array}{llll}
u_{1} & u_{2} & \ldots & u_{N}
\end{array}\right]^{T}, \vec{u} \in \mathfrak{R}^{N \times 1}
\end{gathered}
$$

In the above equations $[\ldots]^{T}$ represents the transpose of a matrix. In equation (26), the members of the vector $\vec{u}$, could be the link offset $(r)$ for the DH model or the Hayati angle $(\beta)$ for the Hayati model depending on the chosen configuration of the robot.

$$
u_{i}=r_{i} \text { or } \beta_{i}, i=1, \ldots, N
$$

The homogeneous transformation, $H_{0}^{N}$ can also be written as,

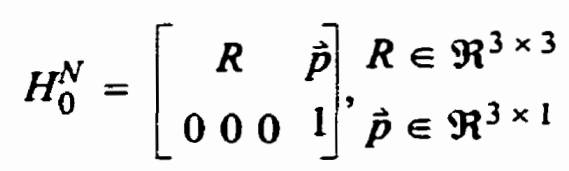

where, $\mathrm{R}$ represents the rotation matrix and $\vec{p}$ represents the end-effector position vector. 
Let, $\vec{x}$ represent the end-effector pose (position, $\vec{p}$ and orientation, $\vec{\rho}$ ). The end-effector position can be known by studying the homogeneous transformation $H_{0}^{N}$ and the orientation $\vec{p}$ can be determined from the rotation matrix, $R$. The end effector pose, $\vec{x}$ can be written as,

$$
\vec{x}=\left[\begin{array}{l}
\vec{p} \\
\vec{\rho}
\end{array}\right]=f(\grave{l}, \vec{\alpha}, \vec{\Theta}, \vec{u})
$$

Thus,

$$
\begin{aligned}
& \vec{p}=f(\grave{l}, \vec{\alpha}, \vec{\Theta}, \vec{u}) \\
& \vec{\rho}=f(\grave{l}, \vec{\alpha}, \vec{\Theta}, \vec{u})
\end{aligned}
$$

The joint angle value, $\Theta_{i}$ is known by reading the optical encoder on the revolute joints of the RoboTwin. The joint encoder reading $\left(\theta_{i}\right)$ is scaled and calibrated (level $I$ or joint level calibration) to provide the joint angle value $\left(\Theta_{i}\right)$ by the following equation.

$$
\Theta_{i}=\theta_{i}+\delta \theta_{i}
$$

where, $\delta \theta_{i}$ is the joint angle offset. Due to the high accuracy of the optical encoders, the joint encoder reading can be known exactly. Any error in the joint angle value would have to come from the joint offset. Thus, the joint offset is included in the parameters to be determined. The parameters to be determined for each link $q_{i}$, can be written as,

$$
\left[\vec{q}_{j} \vec{q}_{j+1} \vec{q}_{j+2} \vec{q}_{j+3}\right]^{T}=\left[l_{i} \alpha_{i} u_{i} \delta \theta_{i}\right]^{T}, j=4 i-3, i=1, \ldots, N
$$

The unknown parameter vector, $\vec{q}$ can be represented as,

$$
\vec{q}=\left[\begin{array}{llll}
q_{1} & q_{2} & \ldots & q_{4 N}
\end{array}\right]^{T}, \vec{q} \in \mathfrak{R}^{4 N \times 1}
$$

The joint encoder reading vector, $\vec{\theta}$ can be written as,

$$
\vec{\theta}=\left[\begin{array}{llll}
\theta_{1} & \theta_{2} & \ldots & \theta_{N}
\end{array}\right]^{T}, \vec{\theta} \in \Re^{N \times 1}
$$


From equations (33), (33), (34) and (35), the forward model in equation (30) can be rewritten as,

$$
\vec{p}=f(\vec{q}, \vec{\theta})
$$

\subsubsection{Measurement}

Being a re-configurable robot, the end-point measurement strategy for the RoboTwin cannot be configuration dependent. Unfortunately, many contact and non-contact measurement techniques used by other researchers are configuration dependent and are designed for a particular robot. Other techniques used by researchers, make use of expensive equipment like vision systems and coordinate measurement machines. These would be unsuitable for the RoboTwin and would conflict with the design criterion established.

Hollerbach's technique [33] of autonomous calibration known as closed-loop kinematic calibration is very attractive for calibrating the RoboTwin as it eliminates the need for an endpoint locating system. This would make the measurement system very inexpensive and independent of configuration. In [33] it is said that if a manipulator is formed into a closed kinematic chain, then its joint angle readings alone are enough to identify the kinematic parameters.

An attempt was made to implement this approach using a closed-loop kinematic calibration on the RoboTwin. The RoboTwin was classified as having one of two possible configurations: dual-arm configuration and single arm configuration. The dual-arm configuration was made into a mobile closed kinematic chain by connecting the two robot arms together using a bracing tool (figure 12). The single arm redundant configuration was made into a mobile closed chain by means of attaching a 3-dof passive joint to the end-effector (figure 12). The 3-dof passive joint was achieved by means of a ball-socket joint as shown in the figure. This mobile closed loop was 

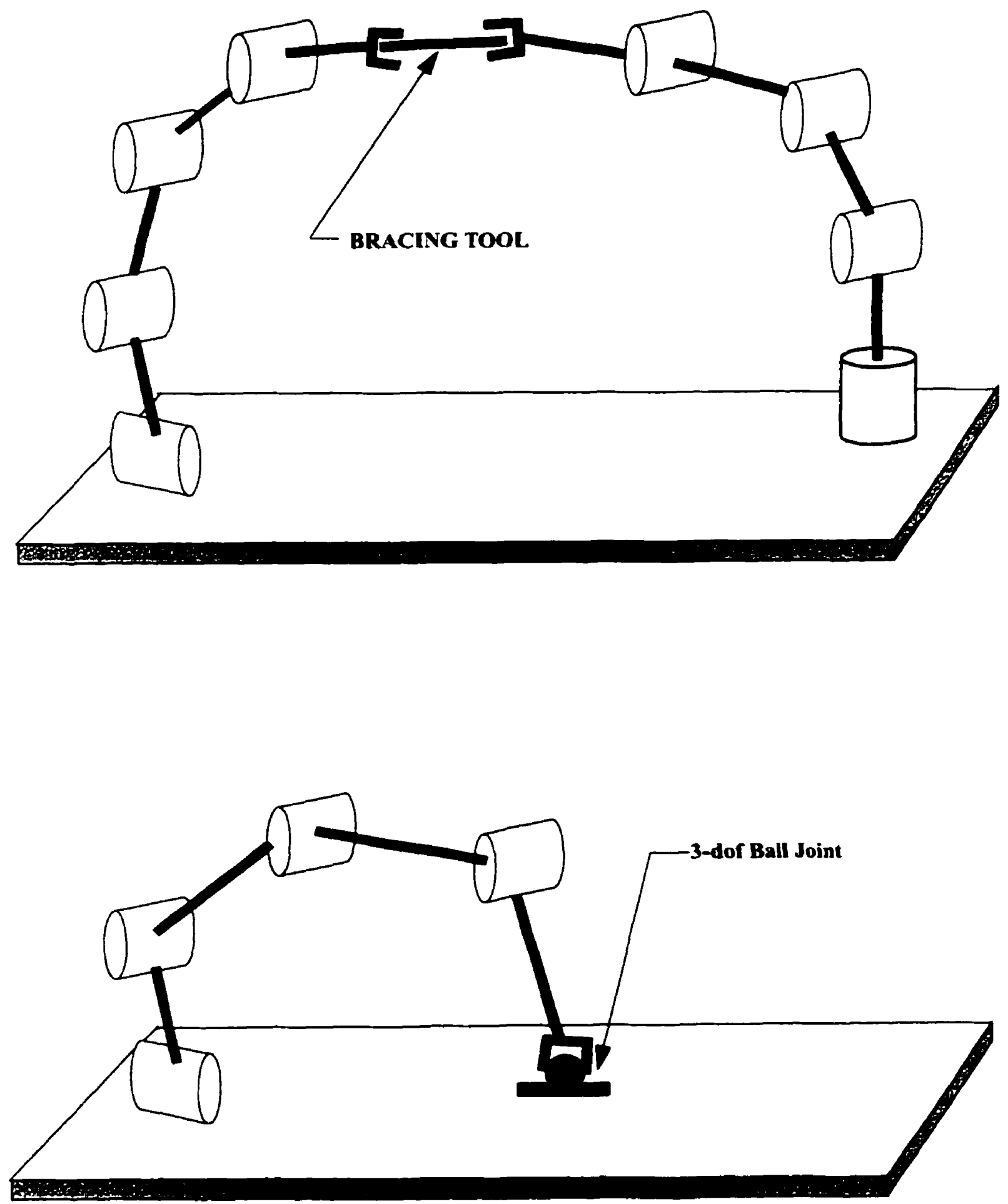

Figure 12. Mobile Closed Kinematic Chain 
then moved to many different postures manually to collect the joint angle data. This data was then used for identification purposesThis approach was unsuccessful due to the small degree of mobility achieved in the mobile closed loop chain. When two robot arms, each with four joints are connected together to form a chain, the degree of mobility achieved was two. When a four jointed robot arm was connected with a passive 3-dof constraint, the degree of mobility achieved was one. Due to the small degree of mobility, the manual motion of the chain for data collection was not propagated to all the robot joints. Depending on the configuration, some joints could become completely immobile. Due to the immobile joints, the Jacobian matrix representing a change in the end effector position with respect to the parameters becomes rank deficient. This led to a singularity during the identification, thus making the parameters of the immobile joint unidentifiable.

The approach was modified to circumvent the problem faced. The idea of using a bracing tool was discarded as there was no way of achieving a higher degree of mobility. Instead, the two arms would be calibrated separately. A greater degree of mobility of closed chain was achieved by using a six degree of freedom passive constraint. Such a constraint was realized by means of a ball-bar mechanism which consisted of two 3-dof ball joints connected at the two ends of a long steel bar. A schematic diagram showing a ball-bar mechanism connected to an arm with three joints is shown in figure 13.

The ball-bar mechanism consists of three components: (i) an adapter socket; (ii) two rod-end bearings; (iii) steel bars of different lengths threaded at both ends. An assembly drawing of the ball bar is shown in figure 14. The drawings for the rod end bearing and the adapter socket are shown in figures 15 and 16 respectively

The ball-bar mechanism is connected to the RoboTwin base plate by means of the adapter socket which has internal threads on one end and external threads on the other. The RoboTwin base plate is used for rigid connection between the base joint (first joint) and the work table. It consists of a grid of threaded holes. The external threads of the adapter are screwed into the 


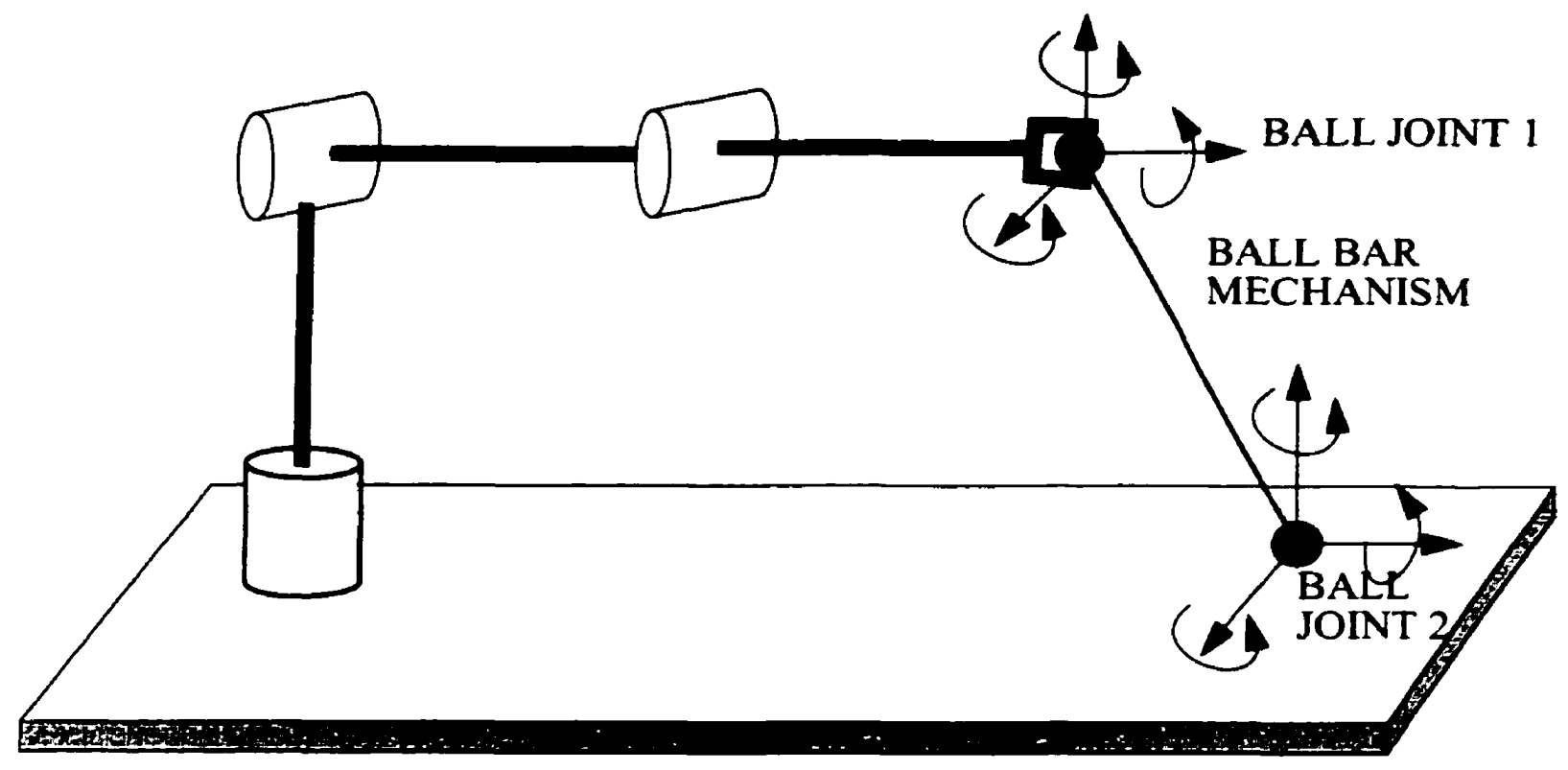

Figure 13. Mobile Closed Kinematic Chain Using a Ball-bar Mechanism

threaded holes of the base plate to provide a rigid connection. The female end of the rod end is screwed into the internal threads of the adapter. The steel bar is connected to the male end of the rod-end. A similar connection between the other rod end and the steel bar is established on the other end of the steel bar. The robot gripper holds to the rod end by means of the gripper and two nuts.

A major advantage of having a ball-bar mechanism that has the flexibility of the mechanism described is the large workspace that can be covered to collect data over. The adapter can be connected to different points on the base plate grid and different lengths of the steel bar can be used to cover a large workspace. Figure 17 shows the workspace covered by two bars of different lengths.

A similar approach was used by Driels [36] who used a 6-dof ball bar to calibrate a PUMA 500. Goswami [19] used a telescopic ball-bar mechanism to calibrate a PUMA robot. However, 


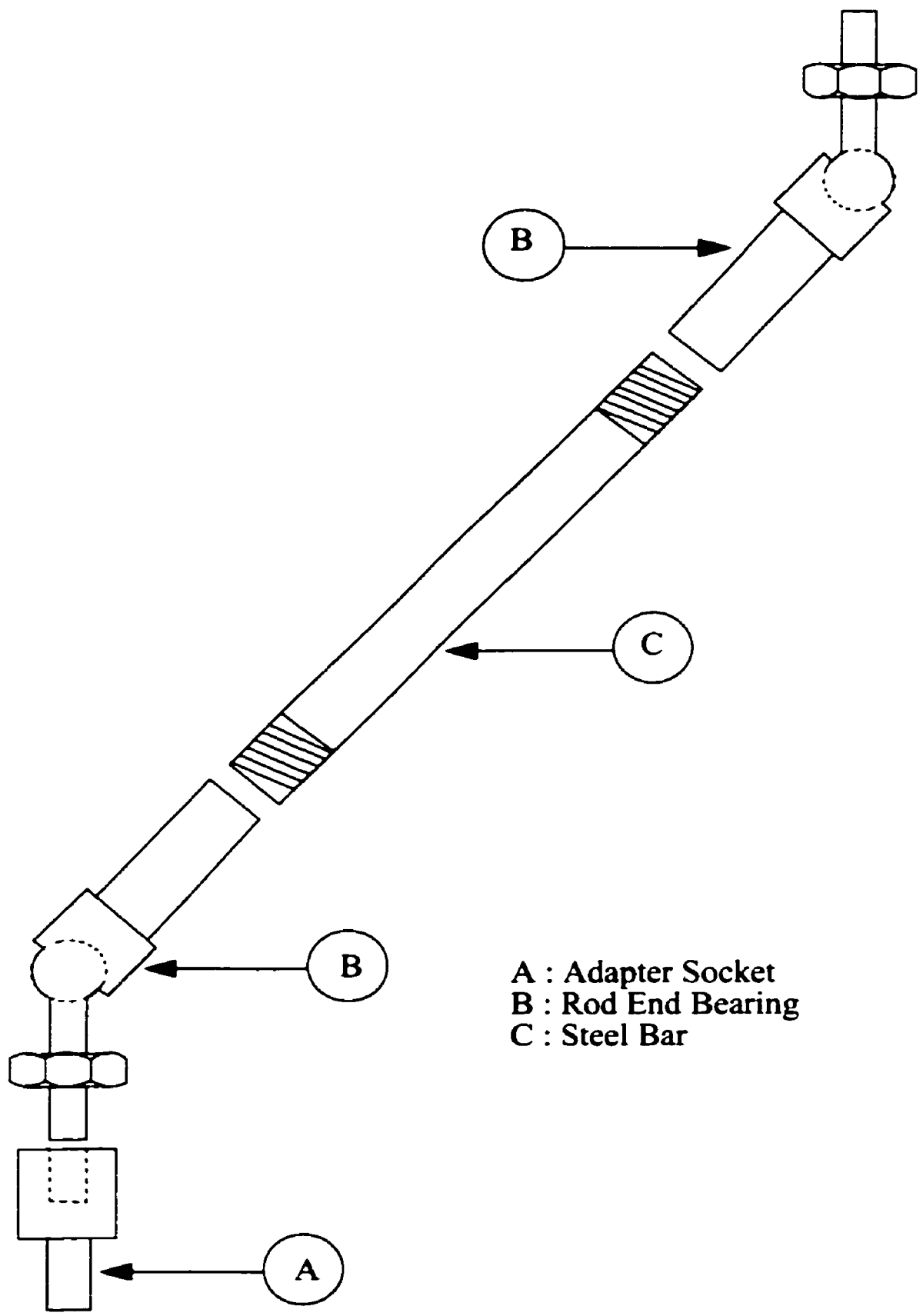

Figure 14. Assembly Drawing of the Ball Bar

the data collection could be done only in a limited workspace. Thus, the results of calibration could not be used in the entire workspace. By using the ball-bar mechanism described earlier, the entire workspace could be covered. 

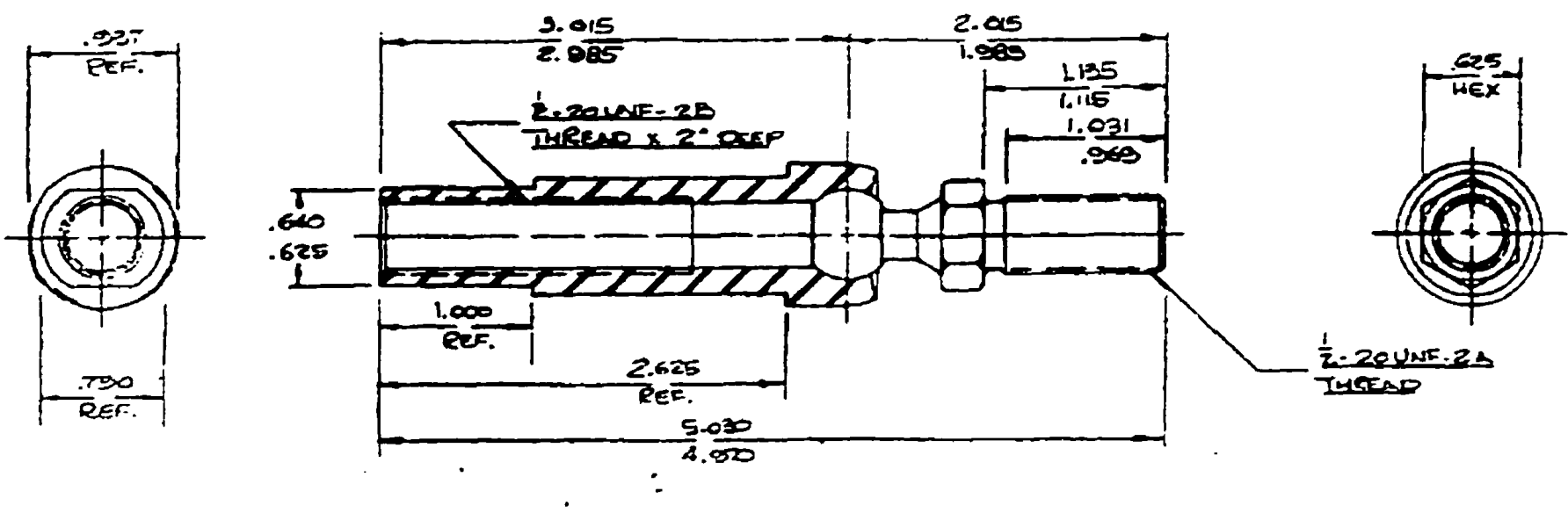

MATEPLLS:

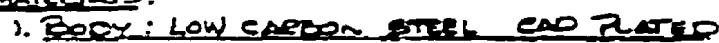

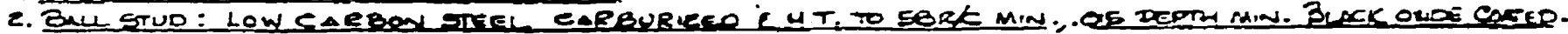
Nores:

1. MGALGNMENT ANGLE: $45^{\circ}$ TOTAL

2. RaOinh CleArance - .0001 $=.002$.

3. maximum ENO Ray . .006. (A)

4. BNe To SWIVEL FREELY N BeOY.

\begin{tabular}{|c|c|c|c|c|c|}
\hline \multicolumn{4}{|c|}{ REVISHONS } & \multirow{2}{*}{ AURORA } & \multirow{2}{*}{ BEARING CO. } \\
\hline & DATE & or & was & & \\
\hline at & 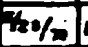 & $\infty$ & Acoes ares $2,0,4$ & ante $12-8-7$ & \multirow{3}{*}{$\begin{array}{l}\text { SWIVEL DEREINGG } \\
\text { ASOEMDLY }\end{array}$} \\
\hline 6 & & & & sene & \\
\hline c) & & & & Daniwn or C.e. & \\
\hline 0 & & & & Aprnoved & SWA-8-1 \\
\hline
\end{tabular}

Figure 15. Rod End Bearing

Joint angle data was collected as the closed kinematic chain was moved around manually in the workspace. The robot's nominal geometry was measured with a foot-ruler.

\subsubsection{Identification}

The identification methodology determines the robot parameters using the length of the steel bar as a 'yardstick'. It makes use of the joint angle data collected and the nominal robot parameters. 


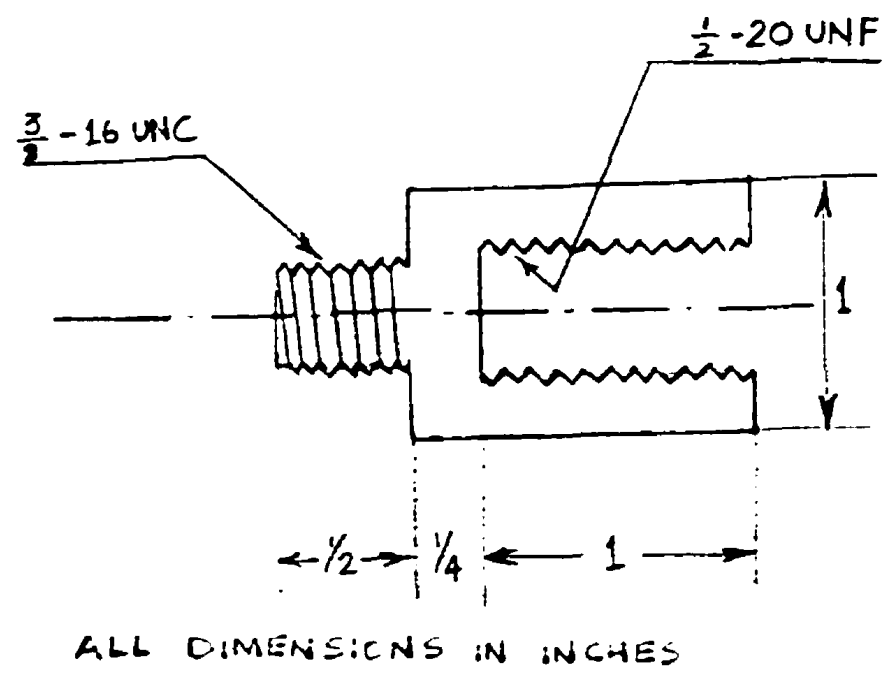

Figure 16. Adapter Socket

Consider a robot and ball-bar mechanism set up as shown in the figure 18. The point $A$ represents the center of the rod end ball joint attached to the base plate and point $B$ represents the center of the rod end ball joint attached to the end-effector. The length vector of the steel bar, $\vec{d}_{A B}$ may be defined by the equation.

$$
\vec{d}_{A B}=\vec{p}_{B}-\vec{p}_{A}
$$

where, $\vec{p}_{B}$ is the position vector to point $B$ (figure 18) in the base frame and $\vec{p}_{A}$ is the position vector to point $A$ (figure 18 ) in the base frame.

The identification methodology uses least square algorithm to minimize the error function given by the following equation.

$$
e=|\vec{e}|=\left|\vec{d}_{A B}^{e}-\vec{d}_{A B}^{n}\right|
$$

where,

$$
\vec{d}_{A B}^{e}=\left.\vec{d}_{A B}\right|_{\vec{q} e}, \text { length vector calculated at exact parameters, } \vec{q}
$$




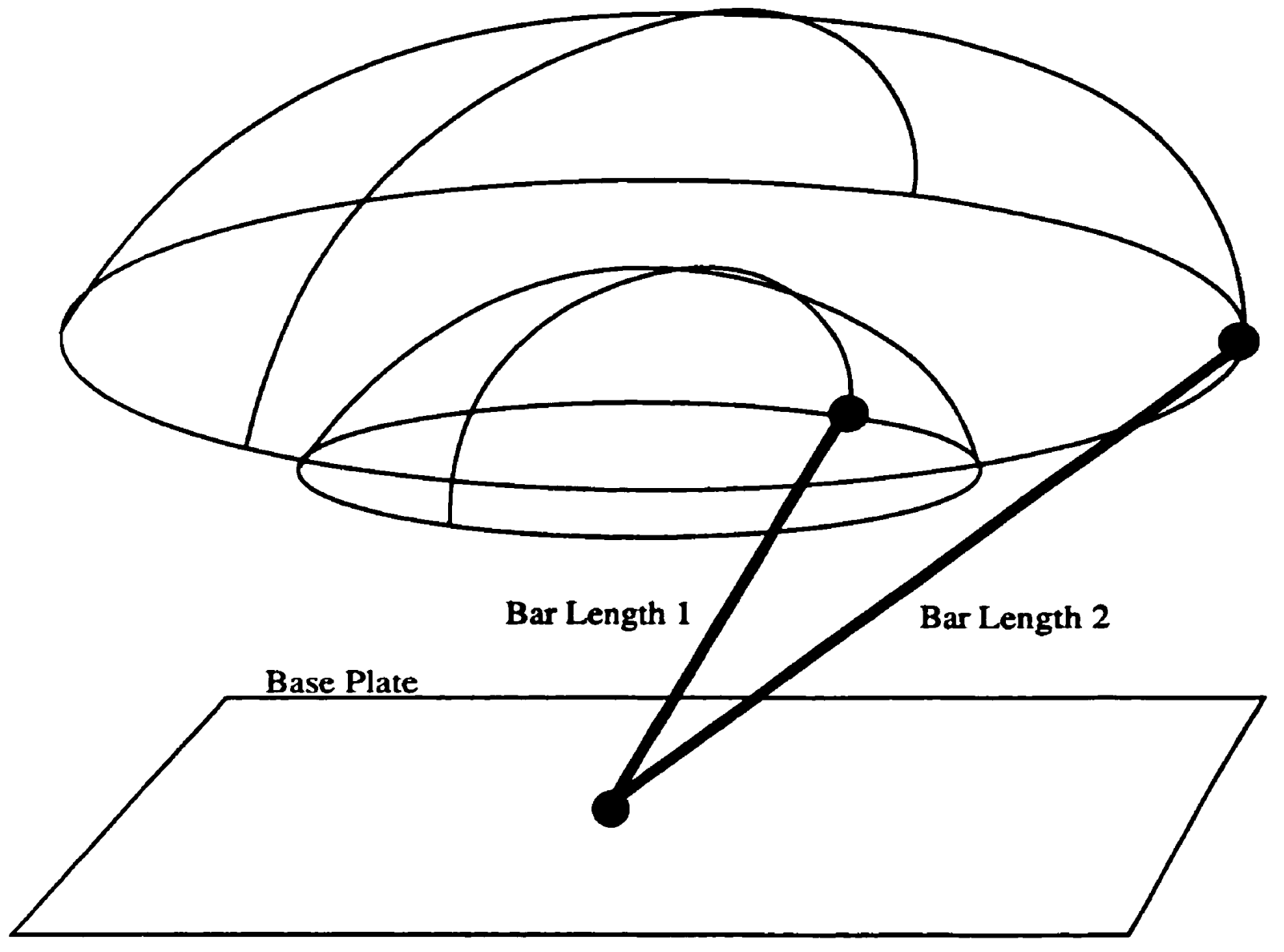

Figure 17. Workspace covered by the Ball-bar mechanism

$$
\vec{d}_{A B}^{n}=\left.\vec{d}_{A B}\right|_{\vec{q}^{*}} \text {, length vector calculated at nominal parameters, } \vec{q}_{n}
$$

and $|*|$ represents the magnitude of the vector.

Equation (36) can be re-written as,

$$
\vec{p}_{B}=f(\vec{q}, \vec{\theta})=f_{x}(\vec{q}, \vec{\theta}) \cdot \dot{i}+f_{y}(\vec{q}, \vec{\theta}) \cdot \dot{j}+f_{z}(\vec{q}, \vec{\theta}) \cdot \vec{k}
$$

where $i, j, \vec{k}$ are the orthogonal unit vectors in the base frame. 


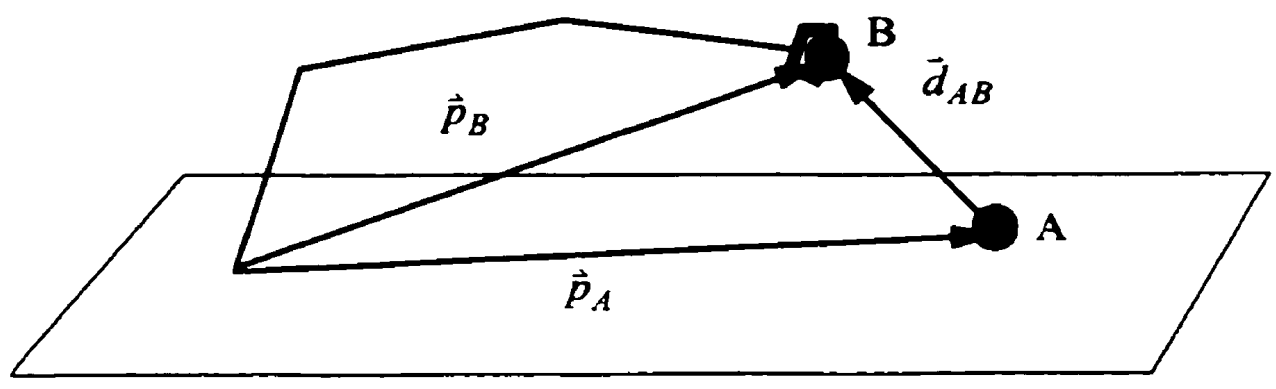

Figure 18. Vector Representation of the Ball-bar in base frame

The vector $\vec{p}_{. A}$ can be written as,

$$
\vec{p}_{A}=x \cdot \grave{i}+y \cdot \dot{j}+z \cdot \vec{k}
$$

Therefore, equation (37) can be re-written as,

$$
\begin{gathered}
\vec{d}_{A B}=\left(f_{x}(\vec{q}, \vec{\theta})-x\right) \cdot \grave{i}+\left(f_{y}(\vec{q}, \vec{\theta})-y\right) \cdot \grave{j}+\left(f_{z}(\vec{q}, \vec{\theta})-z\right) \cdot \vec{k} \\
\vec{d}_{A B}=d_{x} \cdot \grave{i}+d_{y} \cdot \grave{j}+d_{z} \cdot \dot{k}
\end{gathered}
$$

where,

$$
\begin{aligned}
& d_{x}=f_{x}(\vec{q}, \vec{\theta})-x=g_{x}(\vec{q}, \vec{\theta}, x) \\
& d_{y}=f_{y}(\vec{q}, \vec{\theta})-y=g_{y}(\vec{q}, \vec{\theta}, y) \\
& d_{z}=f_{z}(\vec{q}, \vec{\theta})-z=g_{z}(\vec{q}, \vec{\theta}, z)
\end{aligned}
$$

The length of the steel bar, $d$ can be calculated using equation (44),

$$
d=\left|\vec{d}_{A B}\right|=\sqrt{\left(d_{x}\right)^{2}+\left(d_{y}\right)^{2}+\left(d_{z}\right)^{2}}=h\left(d_{x}, d_{y}, d_{z}\right)
$$


The nominal length of the steel bar $d^{n}$, can be determined by using the robot's nominal parameters. The exact length of the steel bar, $d^{e}$, can be approximated using Taylor's series expansion in the neighborhood of its nominal length, $d^{n}$ as shown,

$$
\begin{array}{r}
d^{e}=d^{n}+\left(\left.\frac{\partial h}{\partial d_{x}}\right|_{\hat{q}^{n}} \cdot \Delta d_{x}+\left.\frac{\partial h}{\partial d_{y}}\right|_{\hat{q}^{n}} \cdot \Delta d_{y}+\left.\frac{\partial h}{\partial d_{z}}\right|_{\vec{q}^{n}} \cdot \Delta d_{z}\right)+ \\
\left(\left.\frac{\partial^{2} h}{\partial d_{x}}\right|_{\vec{q}^{n}} \cdot \Delta d_{x}^{2}+\left.\frac{\partial^{2} h}{\partial d_{y}}\right|_{\vec{q}^{n}} \cdot \Delta d_{y^{n}}^{2}+\left.\frac{\partial^{2} h}{\partial d}\right|_{\vec{q}^{n}} \cdot \Delta d_{z}^{2}\right)+\ldots
\end{array}
$$

As $\vec{q}^{n}$ is very close to $\vec{q}^{e}$, the second order error terms can be ignored, thus

$$
d^{e}=d^{n}+\left(\left.\frac{\partial h}{\partial d_{x}}\right|_{\hat{q}^{n}} \cdot \Delta d_{x}+\left.\frac{\partial h}{\partial d_{y}}\right|_{\vec{q}^{n}} \cdot \Delta d_{y}+\left.\frac{\partial h}{\partial d_{z}}\right|_{\hat{q}^{n}} \cdot \Delta d z\right)
$$

From equation (48),

$$
\frac{\partial h}{\partial d_{x}}=\frac{d_{x}}{d} ; \frac{\partial h}{\partial d_{y}}=\frac{d_{y}}{d} ; \frac{\partial h}{\partial d_{z}}=\frac{d_{z}}{d}
$$

Thus equation (50) becomes,

$$
e=\Delta d=d^{e}-d^{n}=\left.\frac{d_{x}}{d}\right|_{\grave{q}^{n}} \cdot \Delta d_{x}+\left.\frac{d_{y}}{d}\right|_{\grave{q}^{n}} \cdot \Delta d_{y}+\left.\frac{d z}{d}\right|_{\grave{q}^{n}} \cdot \Delta d z
$$

This can be re-written as,

$$
e=\frac{d_{x}^{n}}{d^{n}} \cdot \Delta d_{x}+\frac{d_{y}^{n}}{d^{n}} \cdot \Delta d_{y}+\frac{d_{z}^{n}}{d^{n}} \cdot \Delta d_{z}
$$

As the joint encoder readings can be read accurately and the position of point $\mathrm{A}$ is known exactly, the first order Taylor series approximation of equations (45), is

$$
\Delta d_{x}=d_{x}^{e}-d_{x}^{n}=\left.\frac{\partial g_{x}}{\partial \vec{q}}\right|_{\vec{q}^{n}} \cdot \cdot \Delta \vec{q}
$$


where, $d_{x}^{e}$ is the exact value of $d_{x}$ and $d_{x}^{n}$ is the value of $d_{x}$ based on the nominal robot model. Therefore equation (54) can be rewritten as,

$$
\Delta d_{x}=\left.\frac{\partial g_{x}}{\partial \vec{q}}\right|_{\vec{q}^{n}} \cdot \Delta \vec{q}=\left.\frac{\partial f_{x}}{\partial \vec{q}}\right|_{\vec{q}^{n}} \cdot \Delta \vec{q}=\left(\left.\frac{\partial f_{x}}{\partial q_{1}}\right|_{q_{1}^{n}}+\left.\frac{\partial f_{x}}{\partial q_{2}}\right|_{q_{2}^{n}}+\ldots+\left.\frac{\partial f_{x}}{\partial q_{4 N}}\right|_{q_{\sharp . .}^{n}}\right) \cdot \Delta \vec{q}
$$

Similarly,

$$
\begin{aligned}
& \Delta d_{y}=\left.\frac{\partial g_{y}}{\partial \vec{q}}\right|_{\vec{q}^{n}} \cdot \Delta \vec{q}=\left.\frac{\partial f_{y}}{\partial \vec{q}}\right|_{\vec{q}^{n}} \cdot \Delta \vec{q}=\left(\left.\frac{\partial f_{y}}{\partial q_{1}}\right|_{q_{1}^{n}}+\left.\frac{\partial f_{y}}{\partial q_{2}}\right|_{q_{z}^{n}}+\ldots+\left.\frac{\partial f_{y}}{\partial q_{4 N}}\right|_{q_{4 . N}^{n}}\right) \cdot \Delta \vec{q} \\
& \Delta d_{z}=\left.\frac{\partial g_{z}}{\partial \vec{q}}\right|_{\vec{q}^{n}} \cdot \Delta \vec{q}=\left.\frac{\partial f_{z}}{\partial \vec{q}}\right|_{\vec{q}^{n}} \cdot \Delta \vec{q}=\left(\left.\frac{\partial f_{z}}{\partial q_{1}}\right|_{q_{1}^{n}}+\left.\frac{\partial f_{z}}{\partial q_{2}}\right|_{q_{2}^{n}}+\ldots+\left.\frac{\partial f_{z}}{\partial q_{4 N N}}\right|_{q_{t, N}^{n}}\right) \cdot \Delta \vec{q}
\end{aligned}
$$

Equation (53) can be re-written as,

$$
\begin{gathered}
e=\left(\left.\frac{d_{x}^{n}}{d^{n}} \cdot \frac{\partial f_{x}}{\partial \vec{q}}\right|_{\vec{q}^{n}}+\left.\frac{d_{y}^{n}}{d^{n}} \cdot \frac{\partial f_{y}}{\partial \vec{q}}\right|_{\vec{q}^{n}}+\left.\frac{d_{z}^{n}}{d^{n}} \cdot \frac{\partial f_{z}}{\partial \vec{q}}\right|_{\vec{q}^{n}}\right) \cdot \Delta \vec{q} \\
e=K \cdot \Delta \vec{q}
\end{gathered}
$$

where, $e \in \mathfrak{R}^{1 \times 1}, K \in \mathfrak{R}^{1 \times 4 N}, \Delta \vec{q} \in \mathfrak{R}^{4 N \times 1}$

The error in length can also be looked at as a vector, $\vec{e}$

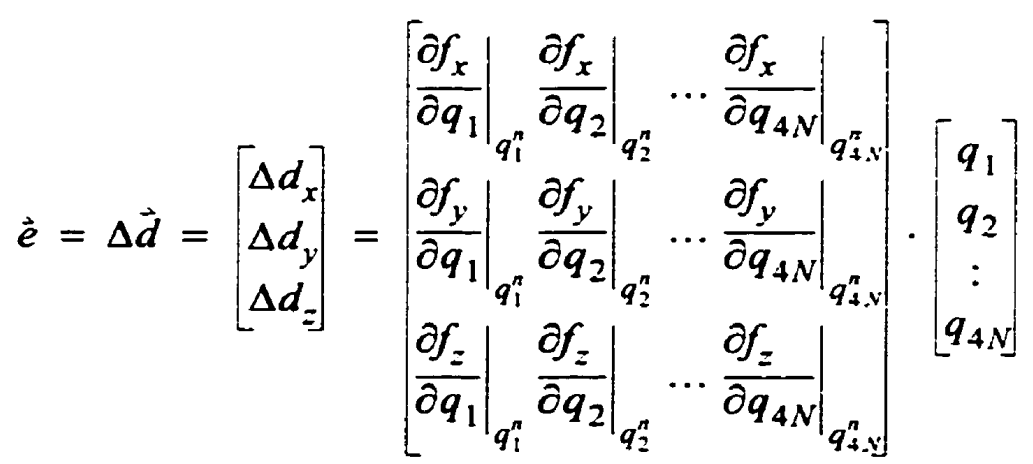




$$
\vec{e}=\left[\begin{array}{llll}
J_{11} & J_{12} & \ldots & J_{1 N} \\
J_{21} & J_{22} & \ldots & J_{2 N} \\
J_{31} & J_{32} & \ldots & J_{3 N}
\end{array}\right] \cdot\left[\begin{array}{c}
q_{1} \\
q_{2} \\
: \\
q_{4 N}
\end{array}\right]=J \cdot \Delta \vec{q}
$$

where $J$ is the Jacobian matrix.

As the error in the bar length occurs only due to the difference in the nominal and actual robot parameters, error in the bar length is the same as the error in end-effector position vector.

$$
\vec{e}=\Delta \vec{d}=\Delta \vec{p}_{B}=J \cdot \Delta \vec{q}
$$

The end-effector position variation $\Delta \vec{p}_{B}$ is the sum of instantaneous linear variations in the link parameters,

$$
\Delta \vec{p}_{B}=\sum_{i=1}^{N}\left(\Delta \vec{p}_{B}\right)_{i}, i=1, \ldots, 4 N
$$

where, $\left(\Delta \vec{p}_{B}\right)_{i}$ is the contribution of the link parameter $i$ to the end effector linear variation.

Consider a link represented using the D-H convention. Let the vector $\vec{b}_{i+1}$ represent a vector from the origin of frame $i$ to the end-effector and the vector $\vec{b}_{i}$ represent a vector from the origin of frame $(i-l)$ to the end-effector. An instantaneous variation in the link offset, $\Delta r_{i}$ contributes to the linear end-effector variation in the direction of $\hat{z}_{i-1}$. An instantaneous variation in the link length, $\Delta l_{i}$ contributes to the linear end-effector variation in the direction of $\vec{x}_{i}$. Similarly an instantaneous variation in the joint angle, $\Delta(\delta \theta)$ contributes to the linear end-effector variation in the direction of $\vec{z}_{i-1} \times \vec{b}_{i}$ where $(x)$ represents the cross product. An instantaneous variation in the twist angle $\Delta \alpha$ contributes to end-effector variation in the direction of $\dot{z}_{i} \times \vec{b}_{i+1}$.

The linear end-effector variation of a robot represented only by $\mathrm{D}-\mathrm{H}$ parameters can be written as, 


$$
\vec{e}=\Delta \vec{p}_{B}=\sum_{i=1}^{N}\left(\Delta r_{i} \cdot \vec{z}_{i-1}+\Delta l_{i} \cdot \vec{x}_{i}+\vec{z}_{i-1} \times \vec{b}_{i} \cdot \Delta(\delta \theta)+\vec{x}_{i} \times \vec{b}_{i+1} \cdot \Delta \alpha_{i}\right)
$$

The Jacobian columns for a link $i$ represented by D-H convention can be written as,

$$
J_{r i}=\left(\Delta r_{i} \cdot \hat{z}_{i-1}\right) ; J_{l i}=\left(\Delta l_{i} \cdot \vec{x}_{i}\right) ; J_{\theta i}=\left(\hat{z}_{i-1} \times \vec{b}_{i} \cdot \Delta(\delta \theta)\right) ; J_{\alpha i}=\left(\vec{x}_{i} \times \vec{b}_{i+1} \cdot \Delta \alpha_{i}\right)
$$

Consider a link represented using the Hayati convention. Let the vector $\vec{b}_{i+1}$ represent a vector from the origin of frame $i$ to the end-effector and the vector $\vec{b}_{i}$ represent a vector from the origin of frame (i-l) to the end-effector. An instantaneous variation in the joint angle. $\Delta(\delta \theta)$ contributes to the linear end-effector variation in the direction of $\vec{z}_{i-1} \times \vec{b}_{i}$. An instantaneous variation in the link length, $\Delta l_{i}$ contributes to the linear end-effector variation in the direction of $\vec{x}^{\prime}{ }_{i}$. An instantaneous variation in the twist angle $\Delta \alpha$ contributes to end-effector variation in the direction of $\vec{x}_{i}{ }_{i} \times \vec{b}_{i+1}$. An instantaneous variation in the Hayati angle, $\Delta \beta_{i}$ contributes to the linear end-effector variation in the direction of $\vec{y}_{i} \times \vec{b}_{i+1}$.

The linear end-effector variation of a robot represented only by Hayati parameters can be written as,

$$
\Delta \vec{p}_{B}=\sum_{i=1}^{n}\left(\hat{z}_{i-1} \times \vec{b}_{i} \Delta \delta \theta\right)+\left(\vec{x}_{i} \cdot \Delta l_{i}\right)+\left(\vec{x}_{i}{ }_{i} \times \vec{b}_{i+1} \Delta \alpha_{i}\right)+\left(y_{i} \times \vec{b}_{i+1} \Delta \beta_{i}\right)
$$

From figure $11, \dot{x}_{i}^{\prime}$ can be written as,

$$
\vec{x}_{i}{ }_{i}=C \theta_{i} \cdot \vec{x}_{i-1}+S \theta_{i} \cdot \vec{y}_{i-1}
$$

Equation (66) can be re-written as,

$$
\begin{gathered}
\Delta \vec{p}_{B}=\sum_{i=1}^{n}\left(\hat{z}_{i-1} \times \vec{b}_{i} \Delta \delta \theta\right)+\left(\left(C \theta_{i} \cdot \vec{x}_{i-1}+S \theta_{i} \cdot \vec{y}_{i-1}\right) \Delta l_{i}\right)+ \\
\left(\left(C \theta_{i} \cdot \vec{x}_{i-1}+S \theta_{i} \cdot \vec{y}_{i-1}\right) \times \vec{b}_{i+1} \Delta \alpha_{i}\right)+\left(y_{i} \times \vec{b}_{i+1} \Delta \beta_{i}\right)
\end{gathered}
$$


The Jacobian columns for a link represented by Hayati convention can be written as,

$$
\begin{gathered}
J_{\beta i}=\left(y_{i} \times \vec{b}_{i+1} \Delta \beta_{i}\right) ; J_{l i}=\left(C \theta_{i} \cdot \vec{x}_{i-1}+S \theta_{i} \cdot \vec{y}_{i-1}\right) \Delta l_{i} \\
J_{\theta i}=\left(\dot{z}_{i-1} \times \vec{b}_{i} \cdot \Delta(\delta \theta)\right) ; J_{\alpha i}=\left(C \theta_{i} \cdot \vec{x}_{i-1}+S \theta_{i} \cdot \vec{y}_{i-1}\right) \times \vec{b}_{i+1} \Delta \alpha_{i}
\end{gathered}
$$

The row matrix, $\mathrm{K}$ (refer to equation (59)), using the entries in the Jacobian matrix, can be used to determine the value of $e$.

The process of data collection was done by manually moving the robot over the whole workspace into many postures. The $\mathrm{K}$ matrix and the $e$ value were determined for every posture and arranged as shown,

$$
E=\left[\begin{array}{c}
e^{1} \\
e^{2} \\
: \\
e^{m}
\end{array}\right] ; \quad M=\left[\begin{array}{c}
K^{1} \\
K^{2} \\
: \\
K^{m}
\end{array}\right]
$$

Equation (59) can be re-written in general as,

$$
E=M \cdot \Delta \vec{q}
$$

where, $E \in \mathfrak{R}^{m \times 1}, M \in \mathfrak{R}^{1 \times N}, \Delta \vec{q} \in \mathfrak{R}^{N \times 1}$

By linear least squares method, $E$ can be minimized when

$$
\Delta \vec{q}=\left(M^{T} \cdot M\right)^{-1} \cdot M^{T} \cdot E
$$

The error in parameters, $\Delta \vec{q}$ is used to update the nominal parameter estimate and obtain the corrected parameters.

In equation (59), the expression for the error in the bar length, $e$, was linearized by ignoring the second order error terms. The linearization error can be removed by using the identification algorithm iteratively until the error, $\Delta \vec{q}$ becomes small or less than some predefined coefficient. The parameter values after each iteration, $\vec{q}^{j+1}$, can be written as, 


$$
\vec{q}^{j+1}=\vec{q}^{j}+\Delta \vec{q}^{j}
$$

where, $\Delta \vec{q}^{j}$ represents the correction in the $j$ th iteration and $\vec{q}^{j}$ represents the parameter values before the $j t h$ iteration.

\subsubsection{A summary of the algorithm}

Step 1. Choose and specify a robot configuration.

Step 2. Form a mobile closed kinematic chain using a ball-bar mechanism fixed at a known point on the base plate grid at one end and the robot end-effector at the other end.

Step 3. Manually move the mobile chain into many postures and store the joint encoder data.

Step 4. Change the bar length by using a different steel bar. If all the steel bar lengths have been used go to step 6 .

Step 5. Go to step 3.

Step 6. Change the ball-bar base point location by moving the ball-bar to a different point on the base-plate grid. If the entire workspace is covered, go to step 8.

\section{Step 7. Go to step step 3.}

Step 8. Calculate the magnitude of error in the bar length due to use of nominal parameters for all postures and arrange in a row vector as shown in equation (70). The error for a particular pose, $e^{j}$ where $j=1, \ldots, m$ can be calculated using equations (36), (37) and (38).

Step 9. Calculate the matrix $M$ as shown in equation (70), by calculating the row matrix, $K$ for each posture. $K^{j},(j=1, \ldots, m)$ can be calculated using the entries in the Jacobian matrix as shown in equations (65) and (66). 
Step 10. The error in parameters, $\Delta \vec{q}$ can be calculated using equation (72).

Step 11. If $\Delta \vec{q} \approx 0$, go to step 13 .

Step 12. Update the nominal parameter estimate by using equation (73). Use the new parameter estimate and go to step 8 .

Step 13. Use the values of $\vec{q}$ as the corrected parameters.

\subsubsection{User Interface Design}

A user interface for calibration was designed as a separate module in the existing user interface that controls the RoboTwin. This module can be initiated by selecting option 6 in the control menu. However, it is essential that the robot encoders are initialized before calibration is done. The initialization can be done by option 0 in the control menu.

The calibration module resides on the host CPU and consists of many screens which guide the user in performing the calibration task.

Screen 1 confirms whether or not the encoders are initialized. In case they are not initialized the user can go back to the control menu by pressing ESCAPE.

Screen 2 prompts the user for the robot configuration information. It prompts the user to enter the number of joints in use and the arm on which these are used. The user could specify that both arms are in use by entering 0 . The user is also prompted for information about the sequence in which the joints are wired to the computer. This information can be entered as a list separated by commas and terminated by ENTER. The computer is connected to the robot joints through amplifiers which control the two arms. The information about the sequence can be easily obtained by labels on the amplifiers. All the information collected is stored in variables. 
Screens 3 and 4 deal with information regarding the nominal robot parameters. The identification algorithm requires the nominal D-H parameters. The algorithm is 'intelligent' as it detects the need for Hayati's parameter based on the D-H model. It assumes the nominal model to have a zero Hayati angle nominally for parallel joints. Screen 3 shows a table with the joints as rows and the D-H parameters (length, offset and twist) as the columns. The user is supposed to enter the parameters by moving the respective fields and pressing ENTER. Screen 4 is a confirmation screen where the nominal D-H parameters are shown. The user is prompted for confirmation of data.

Screen 5 deals with the data collection. The user is prompted to enter the length of the ballbar in use and its XY position relative to the robot base frame. This can be entered as a grid location. The user could store the current state of the encoders by pressing F1 key. The user is expected to manually move all the robot joints and collect the encoder data. The user can change the ball-bar length by pressing F2 and entering the new value. The user could also change the base location by pressing F3 and entering the new XY location. After collecting all the required data, the user is prompted for a file name in which the data should be stored. The name of the file defaults to temp.dat. The data is stored in the file for inspection and/or backup.

After the data is collected and stored, the identification routine is launched. The result of the identification is presented in screen 6 . It shows the new corrected parameters. 


\section{Chapter 4}

\section{Implementation of the Calibration \\ Algorithm and Analysis of Results}

The calibration methodology developed in the previous chapter was implemented on two RoboTwin configurations. The results are presented in this chapter.

Two single arm configurations were chosen. The first configuration was chosen so that it could be modelled using the DH convention and did not have any consecutive parallel or nearparallel joint axes. The second configuration chosen had parallel joint axes and was modelled using the Hayati-DH convention. In the following sections, the implementation on the these configurations is explained and the results presented.

\subsection{Implementation on a DH configuration}

The RoboTwin was assembled into a three-jointed single arm configuration. It consisted of three perpendicular joints as shown in figure 19. 


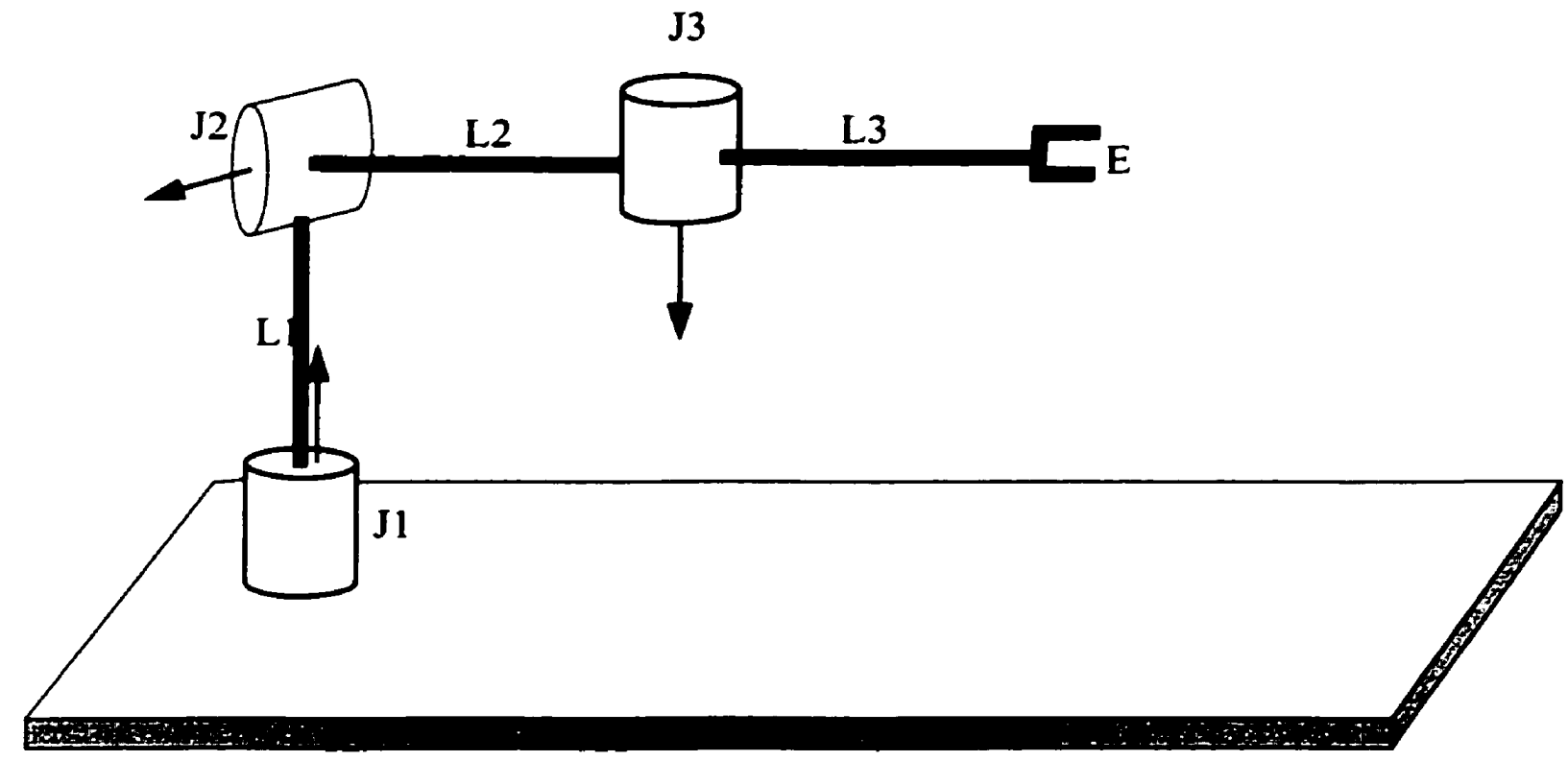

Figure 19. A DH configuration

$\mathrm{J} 1, \mathrm{~J} 2$ and $\mathrm{J} 3$ represent the three revolute joints. $\mathrm{L} 1, \mathrm{~L} 2$ and $\mathrm{L} 3$ represent the three linkages. E represents the end-effector. The first joint $(\mathrm{J} 1)$ is a re-configurable joint configured as a type II joint. The other joint ( $\mathrm{J} 2$ and $\mathrm{J} 3$ ) are type I joints. The joint axes of two consecutive joints are perpendicular.

The RoboTwin was formed into a mobile closed-loop kinematic chain by using a ball-bar mechanism. One end of the ball-bar was held by the gripper while the other end was screwed to the base plate. The hold of the gripper on the ball-bar end was strengthened by means of an extra nut used as shown in figure 20. 


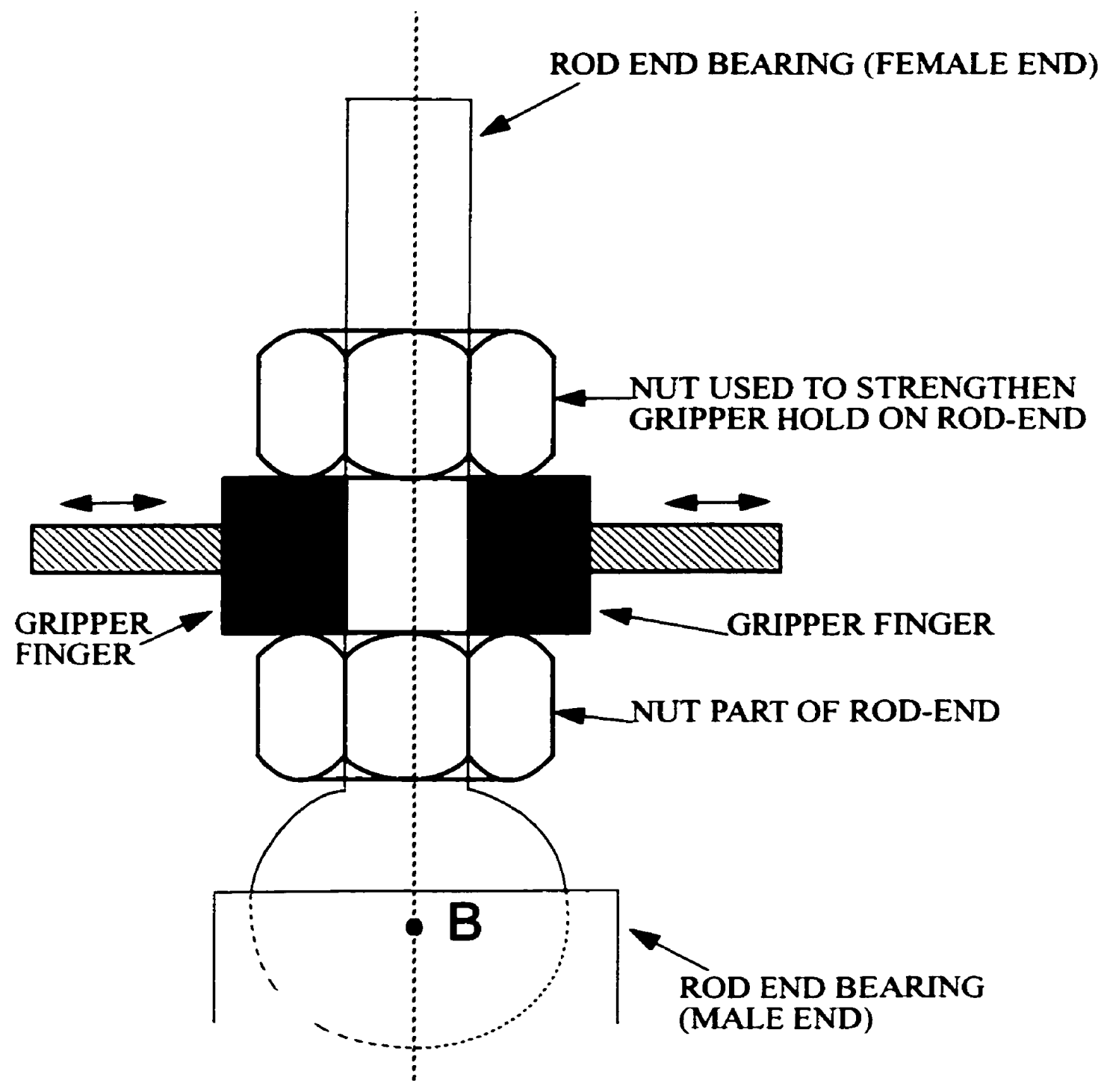

Figure 20. Hold of the gripper on the Bal-bar end

In figure 20 , the point $B$ represents the center of the rod-end ball and is the point about which the rod-end (male end) swivels. 
As the robot does not have any consecutive parallel or near-parallel joints, it was modelled using the DH convention and frames were assigned accordingly. The frame allocation for the mobile chian is shown in figure 21 .

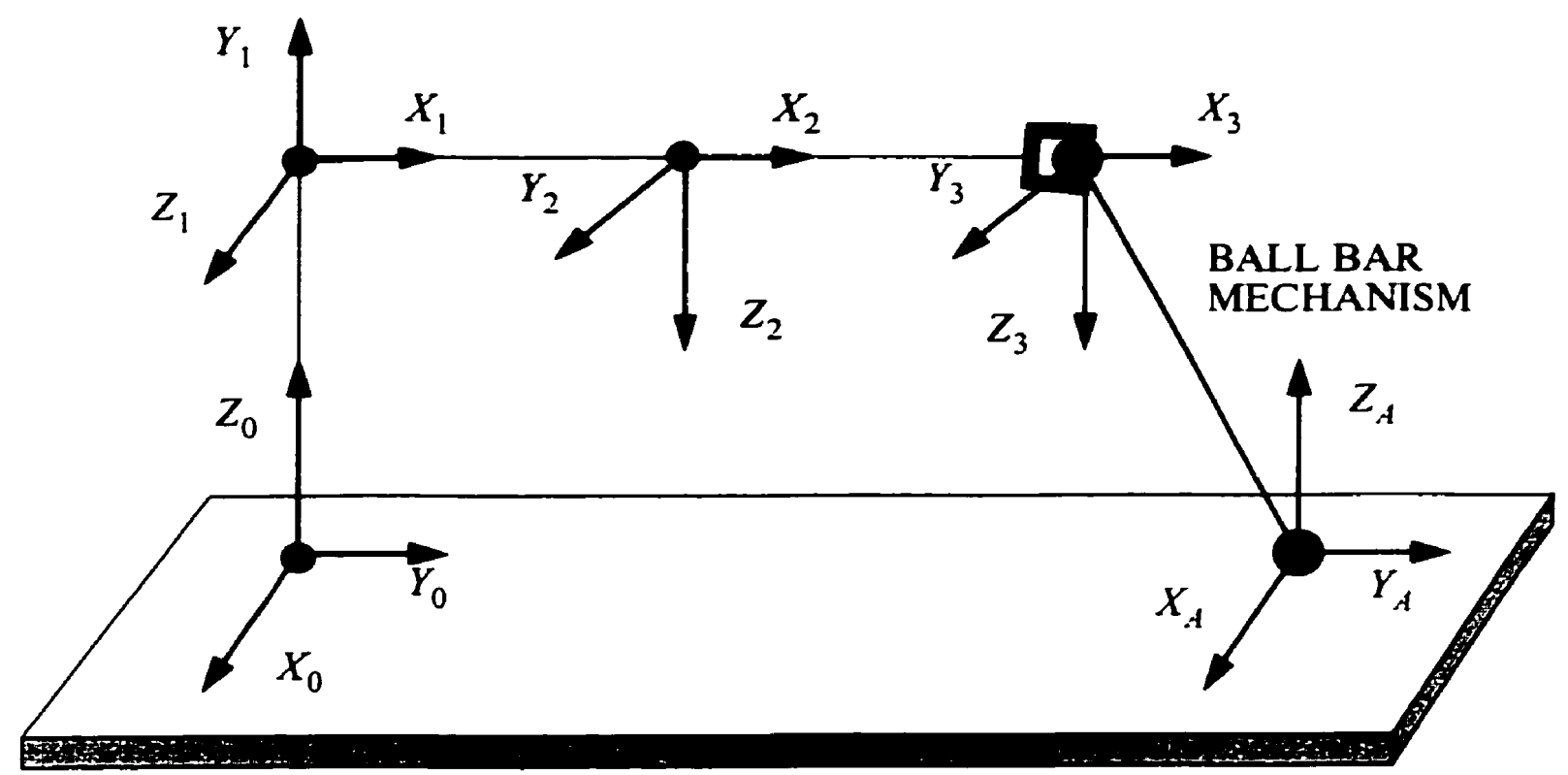

Figure 21. Frame allocation

The base frame $\left(X_{0} Y_{0} Z_{0}\right)$ was assigned arbitrarily. The end-effector frame was located at point $B$ which is shown in figure 20. In figure 21, point A represents the swivel point of the other end of the ball-bar which is screwed to the base plate. The orientation of the frame located at the point $\mathrm{A}\left(X_{A} Y_{A} Z_{A}\right)$ is the same as that of the base frame.

Using the frame allocation, nominal measurements were made on the robot. These were made by marking points on the robot with a marker and measuring the distances using a footruler. These measurements are reported in Table 1. The encoder offsets were nominally assumed to be zero. 
TABLE 1. Nominal Measurements of the Robot

\begin{tabular}{c||cccc}
\hline Link Number & Length (l) in mm $\begin{array}{c}\text { Link Offset }(r) \text { in } \\
\mathrm{mm}\end{array}$ & $\begin{array}{c}\text { Twist Angle }(\alpha) \\
\text { in degrees }\end{array}$ & $\begin{array}{c}\text { Encoder Offset } \\
(\delta \theta) \text { in degrees }\end{array}$ \\
\hline \multirow{2}{*}{$\begin{array}{l}l_{1}=0 \\
2\end{array}$} & $r_{1}=463.55$ & $\alpha_{1}=90^{\circ}$ & $\delta \theta_{1}=0^{\circ}$ \\
3 & $l_{2}=244.475$ & $r_{2}=0$ & $\alpha_{2}=90^{\circ}$ & $\delta \theta_{2}=0^{\circ}$ \\
$l_{3}=244.475$ & $r_{3}=0$ & $\alpha_{3}=90^{\circ}$ & $\delta \theta_{3}=0^{\circ}$
\end{tabular}

The ball-bar mechanism was screwed to a point on the base-plate grid (point A). The location of the point $\mathrm{A}$ was noted by counting the number of holes on the grid in $\mathrm{X}$ and $\mathrm{Y}$ direction relative to the base frame. As the base plate is grid is manufactured to a high tolerance ( 0.002 inch), the location of the point A relative to the base plate is known exactly. Also, the length of the ball-bar is known exactly.

The calibration user-interface was initiated and the nominal robot parameters were entered. The length of the ball-bar and the location of the point $\mathrm{A}$ were also specified. The ball-bar was then moved manually in the workspace. Due to a rigid connection between the ball-bar and the robot, this motion was translated into motion of the robot joints. The joint encoder readings were stored at different points in the workspace by pressing F1 on the keyboard. The length of the ballbar was changed and more readings were stored. The location of the point A was changed and further readings were taken.

The identification algorithm uses one measured point in the workspace to obtain one equation as shown in equation (59). Measurements made at twelve points would provide a system of twelve such equations which would be adequate to solve for the twelve unknown parameters. However, these twelve measurements would not be representative of the entire workspace and the resulting values of the parameters would be valid for those twelve points only. Thus it was 
decided to take measurements at more than the required twelve points in the workspace to obtain an over-determined system of equations.

Due to the relative ease of collecting points, measurements were made at 1440 points in the entire workspace by altering the ball-bar lengths and the location of the point $A$. The data collection process took about two hours. The intention of taking measurements at over fourteen hundred points was to use some of the readings for the identification and the rest for testing the results. Also these 1440 points adequately represented the entire workspace.

Using the 1440 measurement points, four data sets were created. One data set consisted of all the 1440 measurement points. The other three data sets were created by randomly selecting 99 points from the complete data set. The random selection was done using a $\mathrm{C}$ program. The $\mathrm{C}$ program was used thrice to generate the three data sets. The identification algorithm was implemented in MATLAB using all the four data sets.

As only the position related data is used by the algorithm, the parameter $\alpha_{3}$ becomes unidentifiable as it affects only the end-point orientation and not the position. Also, the knowledge of the exact value of $\alpha_{3}$ is not essential to locate the end-point. The parameter $r_{3}$ represents the distance between the centre of the end-effector and the point B (as shown in figure 20) on the rodend. This is known accurately from the drawings and does not need to be identified. The identification algorithm thus identifies the remaining ten parameters. 
The results of identification are presented in Table 2 in the form of identified parameters. Table 2 shows values of identified parameters using each of the four data sets. It also shows the nominal values for comparison.

TABLE 2. Identified Parameters

\begin{tabular}{|c|c|c|c|c|c|c|c|}
\hline Parameter & $\begin{array}{l}\text { Nominal } \\
\text { Value }\end{array}$ & $\begin{array}{c}\text { Using all } \\
1440 \\
\text { points }\end{array}$ & $\begin{array}{l}\text { Using data } \\
\text { set } 1\end{array}$ & $\begin{array}{c}\text { Using data } \\
\text { set } 2\end{array}$ & $\begin{array}{l}\text { Using data } \\
\text { set } 3\end{array}$ & $\mid \begin{array}{c}\text { Range of } \\
\text { variation of } \\
\text { the } \\
\text { identified } \\
\text { parameter }\end{array}$ & $\begin{array}{c}\text { Mean } \\
\text { value of } \\
\text { parameter }\end{array}$ \\
\hline$l_{1}(m m)$ & 0.000 & 1.5844 & 0.1498 & 0.0386 & 1.8925 & 1.8539 & 0.9163 \\
\hline$r_{1}(m m)$ & 463.55 & 472.7512 & 472.4742 & 471.7816 & 471.1253 & 1.6259 & 472.0331 \\
\hline$\alpha_{1}\left(^{\circ}\right)$ & 90.00 & 89.9560 & 90.5104 & 89.7948 & 90.3945 & 0.7156 & 90.1639 \\
\hline$\delta \theta_{1}\left({ }^{\circ}\right)$ & 0.0000 & 0.0421 & -0.5889 & -0.0335 & -0.5959 & 0.6380 & -0.1451 \\
\hline$l_{2}(\mathrm{~mm})$ & 244.475 & 246.1907 & 247.7781 & 245.3099 & 247.6986 & 2.4682 & 246.7443 \\
\hline$r_{2}(\mathrm{~mm})$ & 0.0000 & 0.7441 & -3.3703 & 1.4203 & -4.5821 & 6.0024 & -1.4470 \\
\hline$\alpha_{2}\left(^{\circ}\right)$ & 90.00 & 90.1872 & 89.9678 & 90.4277 & 89.9669 & 0.4608 & 90.1374 \\
\hline$\delta \theta_{2}\left({ }^{\circ}\right)$ & 0.0000 & 0.3676 & 0.3583 & 0.4173 & 0.5924 & 0.2341 & 0.4339 \\
\hline$l_{3}(m m)$ & 244.475 & 238.1572 & 238.3315 & 239.3426 & 236.9069 & 2.4357 & 238.1845 \\
\hline$r_{3}(m m)$ & 31.75 & 31.75 & 31.75 & 31.75 & 31.75 & $\begin{array}{c}\text { Not } \\
\text { Identified }\end{array}$ & 31.75 \\
\hline$\alpha_{3}\left({ }^{\circ}\right)$ & 0.0000 & 0.0000 & 0.0000 & 0.0000 & 0.0000 & $\begin{array}{c}\text { Not } \\
\text { Identified }\end{array}$ & 0.0000 \\
\hline$\delta \theta_{3}\left({ }^{\circ}\right)$ & 0.0000 & -1.0098 & -1.3231 & -1.0001 & -1.0769 & 0.3230 & -1.1024 \\
\hline
\end{tabular}


The identification algorithm converged very fast (usually in two iterations) to a steady value. The results of identification are very close to each other for all the four data sets. This is reflected in the low range of variation amongst the identified parameters.

As a means of checking the success of identification, the identified parameters were used to calculate the error in the bar length at all the 1440 observation points. This error is shown for each data point in figures 22, 24, 26 and 28 . The error in the bar length calculated using the nominal parameters is also shown for comparison. In figure 22, the parameters are identified using data set 1. Figures 24 and 26 show the error in bar length when the parameters were identified using data sets 2 and 3 respectively. Figure 28 uses the parameters identified using all the collected parameters. The frequency distribution of the error in the bar lengths for data sets $1,2,3$ and the complete data set are shown in figures 23, 25, 27 and 29 respectively. 


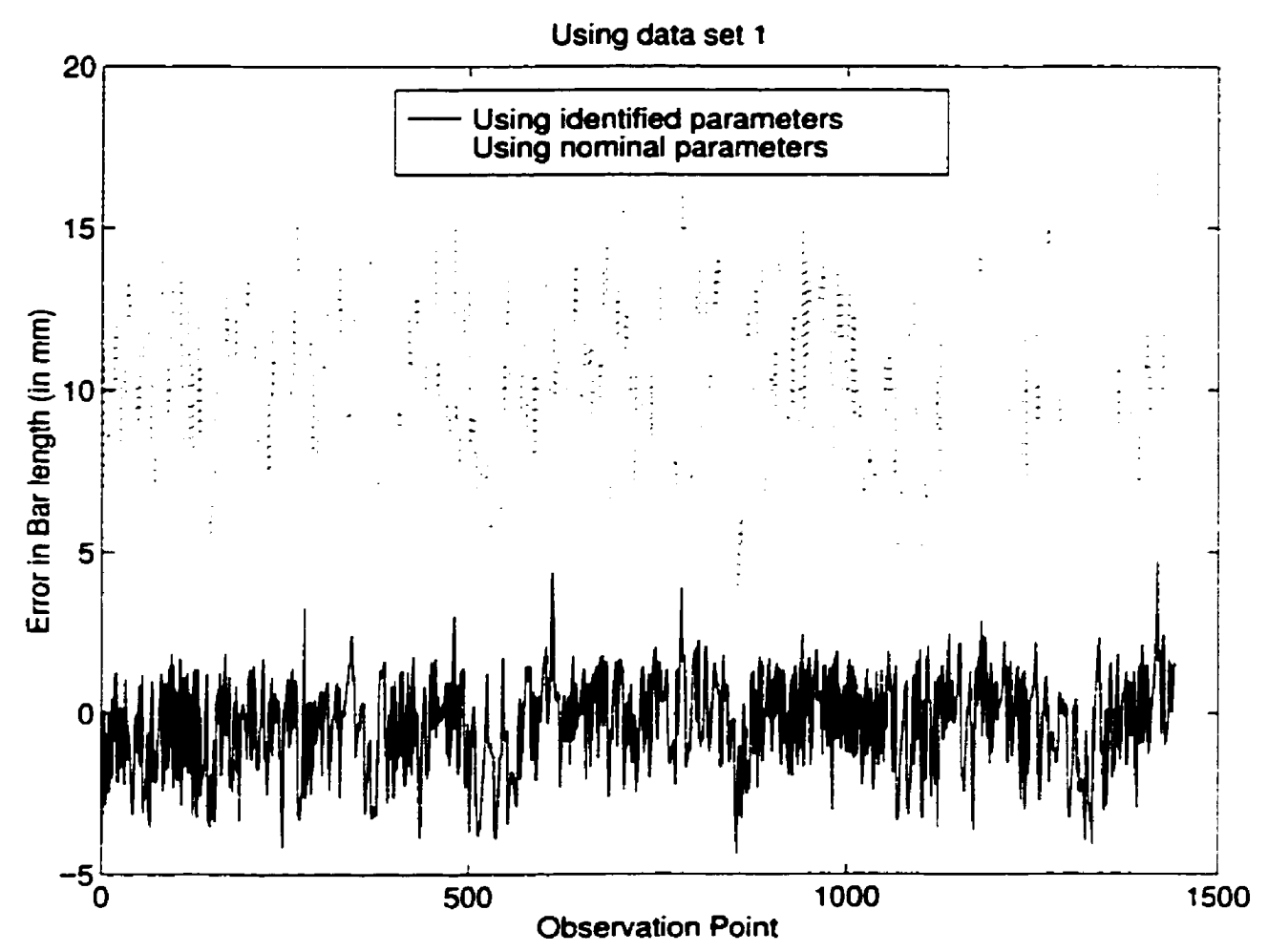

Figure 22. Error in the Bar Length (Identification Using data set 1)

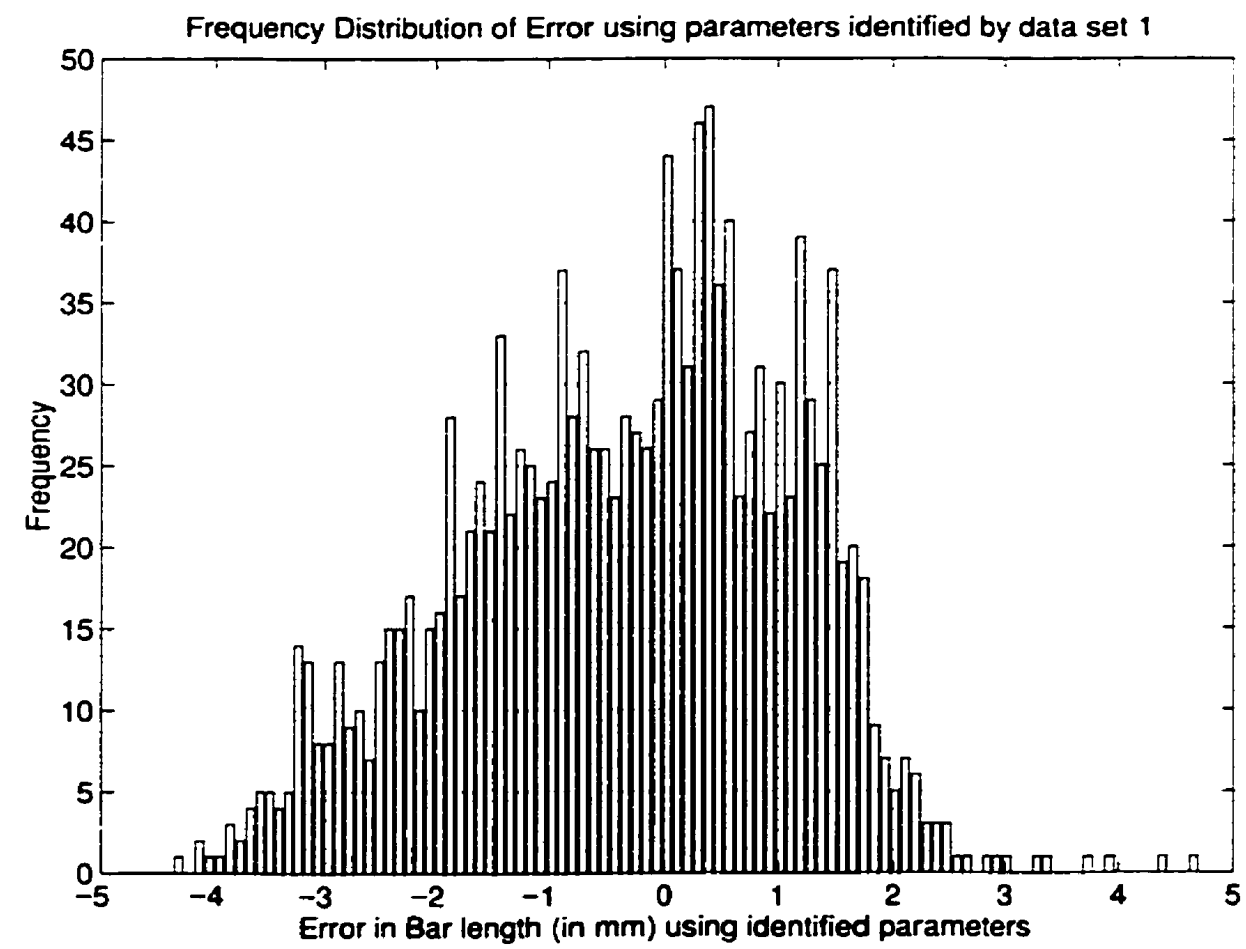

Figure 23. Error Frequency Distribution (Identification using data set 1) 


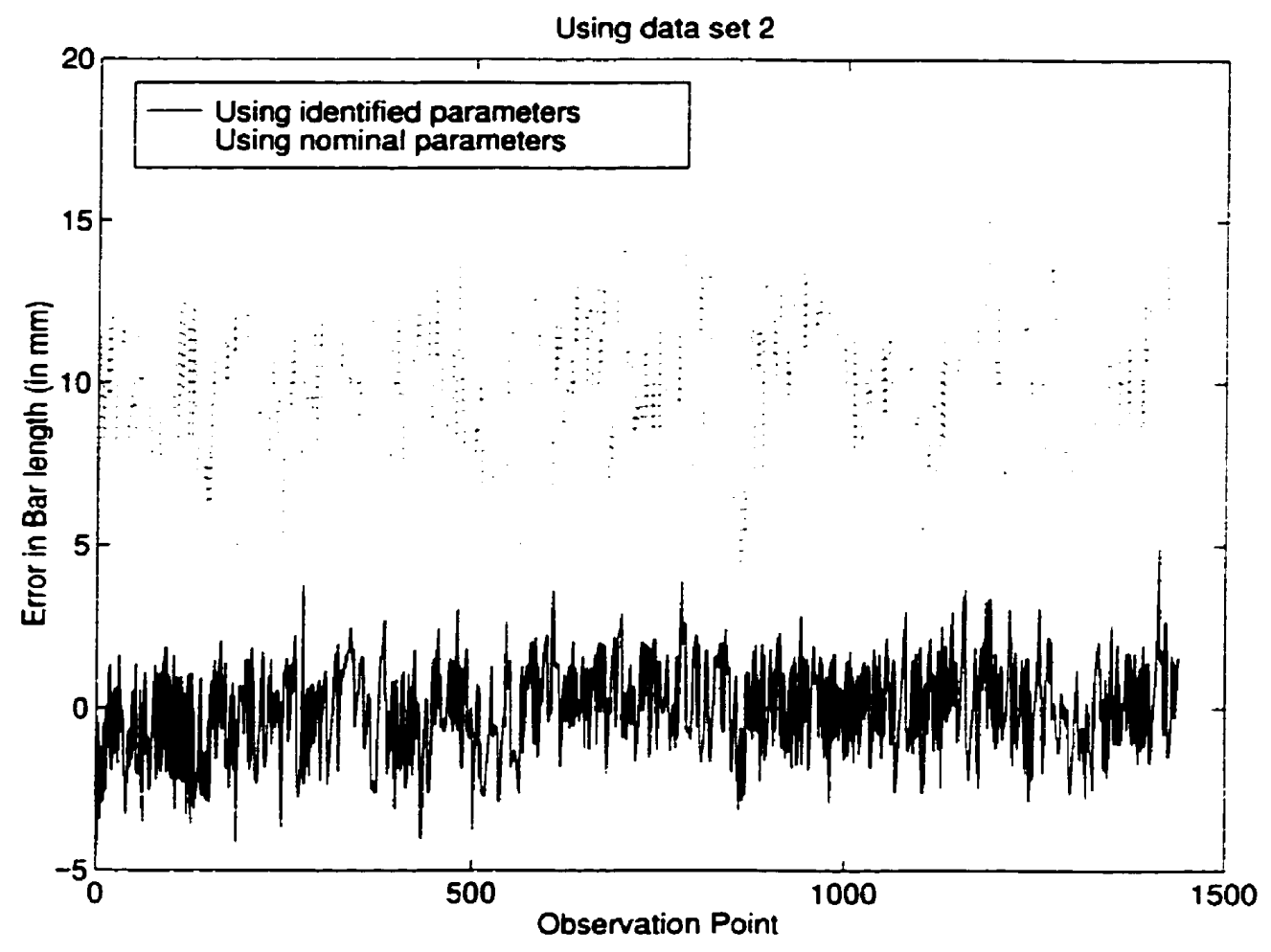

Figure 24. Error in Bar length (Identification Using data set 2)

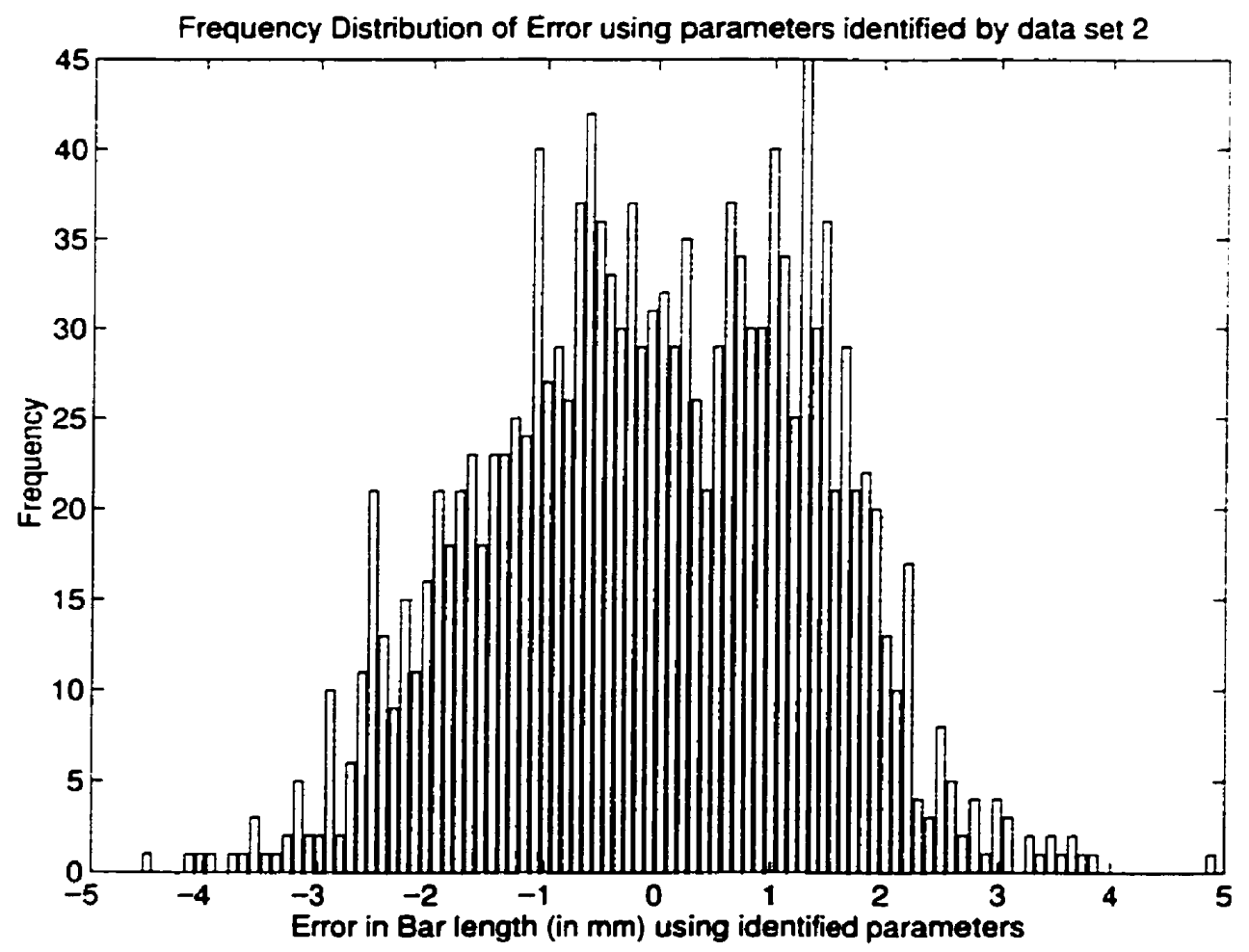

Figure 25. Error Frequency Distribution (Identification Using data set 2) 


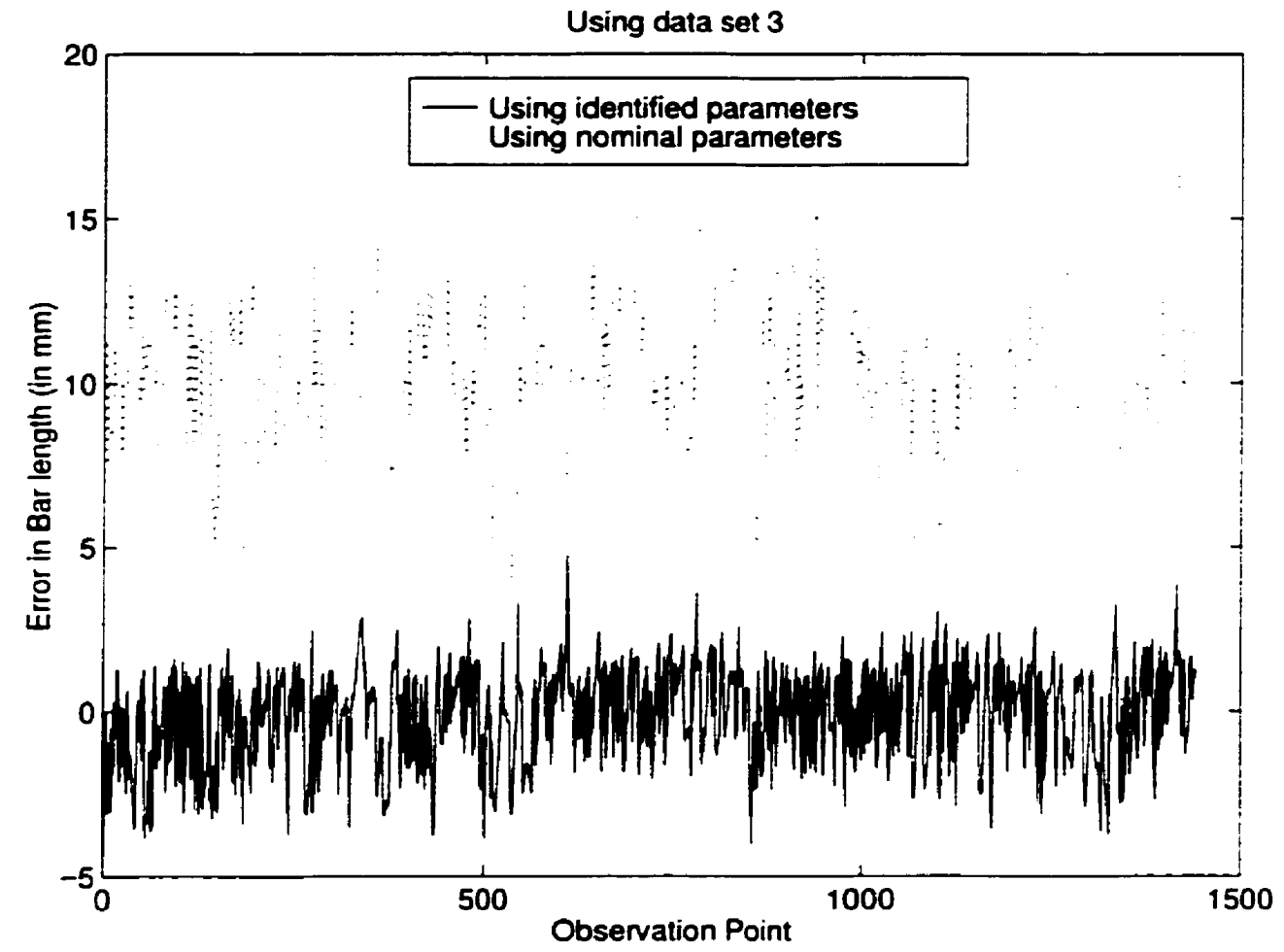

Figure 26. Error in bar length (Identification Using data set 3 )

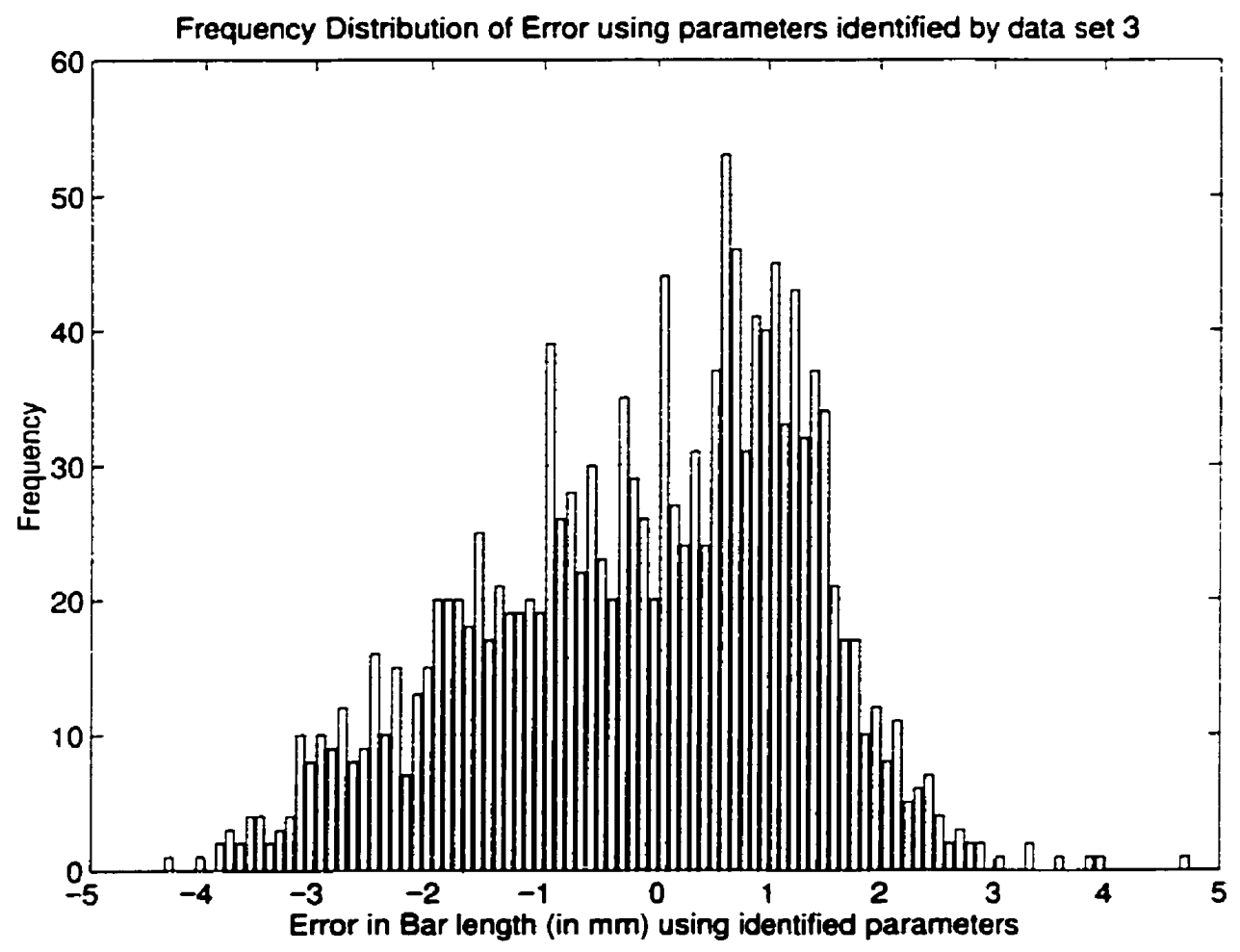

Figure 27. Error Frequency Distribution Using data set 3 


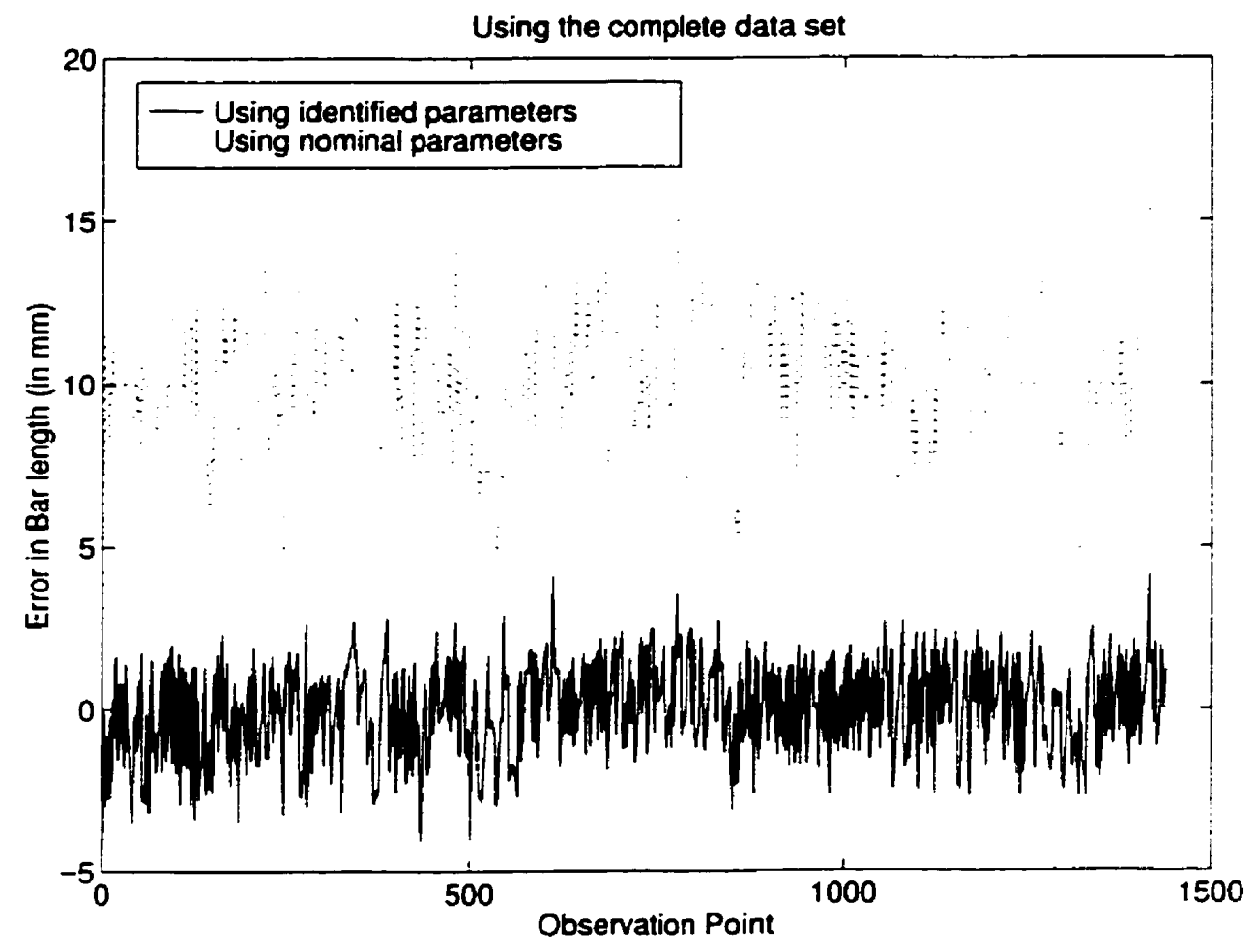

Figure 28. Error in Bar Length (Identification Using the complete data set)

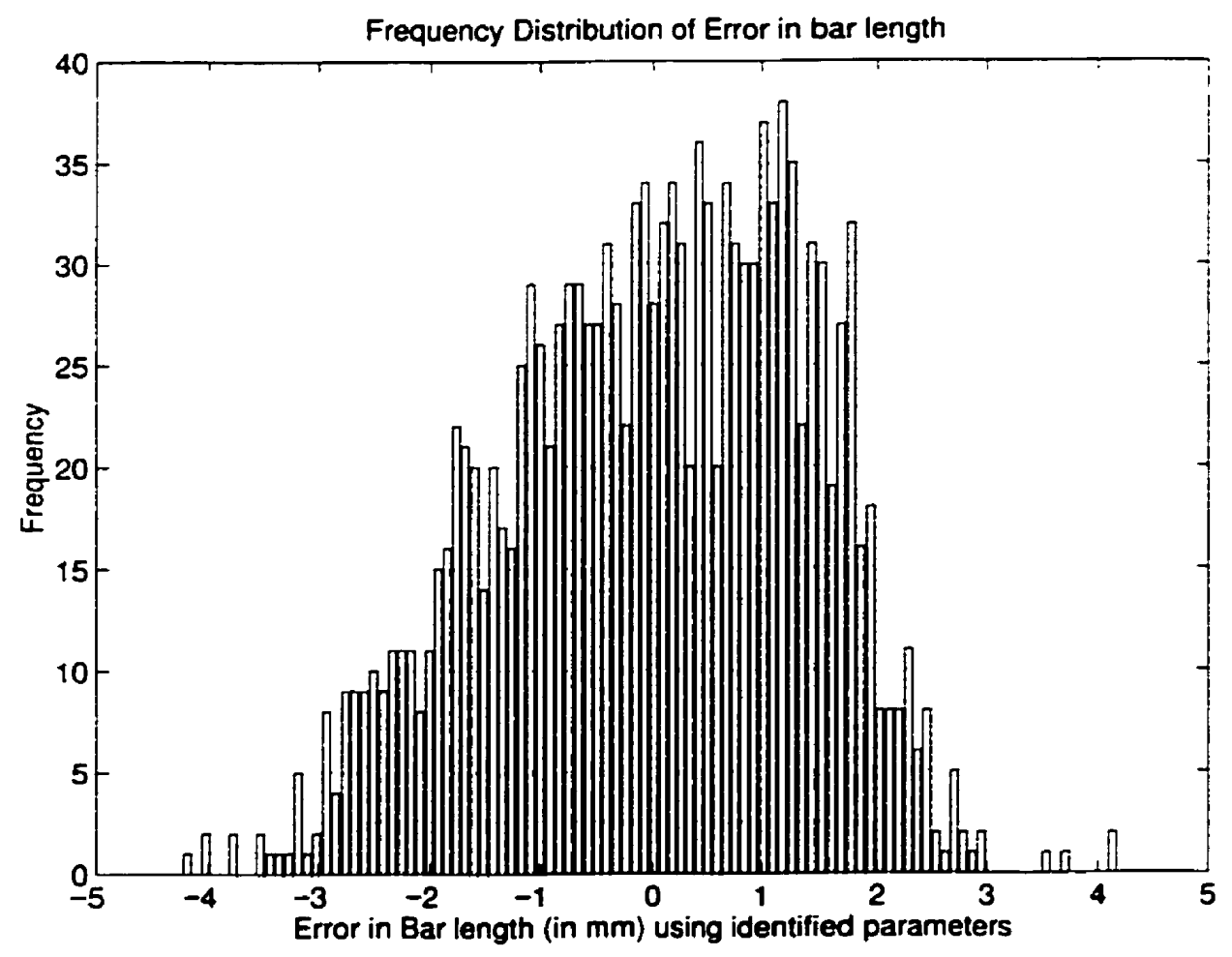

Figure 29. Error Frequency Distribution (Identification Using the Complete Data Set) 
The standard deviation and the mean of these distributions are shown in table 3 . These values are calculated using the identified parameters (table 3) and nominal parameters (table 4).

TABLE 3. Mean and SD of bar length error using identified parameters

\begin{tabular}{c||cccc}
\hline & $\begin{array}{c}\text { Parameters } \\
\text { identified using } \\
\text { data set 1 }\end{array}$ & $\begin{array}{c}\text { Parameters } \\
\text { identified using } \\
\text { data set 2 }\end{array}$ & $\begin{array}{c}\text { Parameters } \\
\text { identified using } \\
\text { data set 3 }\end{array}$ & $\begin{array}{c}\text { Parameters } \\
\text { identified using } \\
\text { the all data points }\end{array}$ \\
\hline Mean & $-0.063 \mathrm{~mm}$ & $-0.0682 \mathrm{~mm}$ & $-0.034 \mathrm{~mm}$ & $-0.04 \mathrm{~mm}$ \\
Standard Deviation & $1.37 \mathrm{~mm}$ & $1.29 \mathrm{~mm}$ & $1.39 \mathrm{~mm}$ & $1.37 \mathrm{~mm}$
\end{tabular}

TABLE 4. Mean and SD of the bar error using nominal parameters

\begin{tabular}{c||c}
\hline Mean & $10.027 \mathrm{~mm}$ \\
Standard Deviation & $3.2812 \mathrm{~mm}$
\end{tabular}

The mean error in the bar length was reduced from approximately $10 \mathrm{~mm}$ to less than 0.063 $\mathrm{mm}$ indicating $95 \%$ improvement in accuracy. The mean value and standard variation for all data are very close to each other indicating that 99 randomly chosen data points in the workspace are enough to produce the same result as 1440 points taken over the entire workspace.

\subsection{Implementation on a Hayati Configuration}

The DH configuration was modified as shown in figure 30 so that two consecutive joints were nominally parallel and a Hayati angle would have to be used to model the robot. 


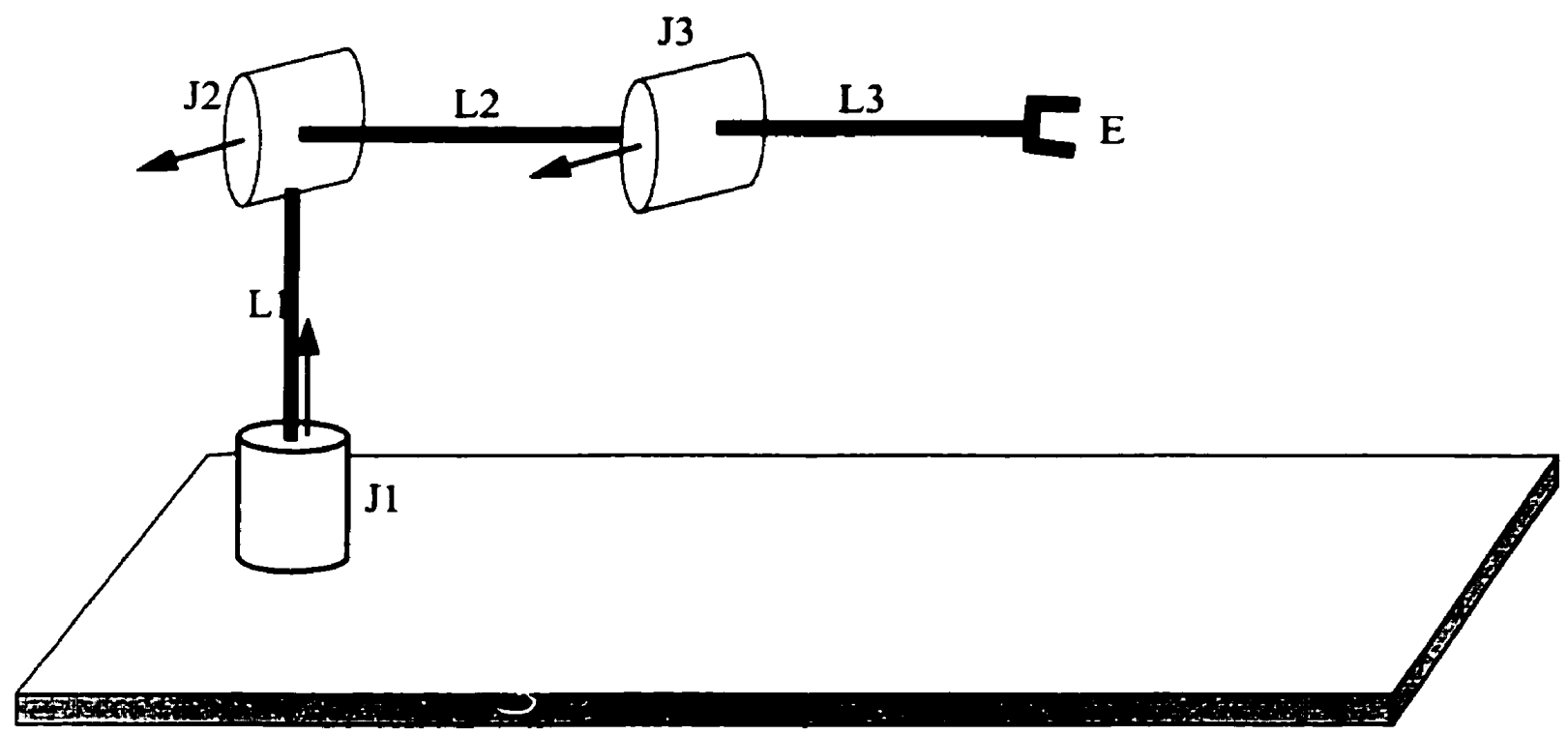

Figure 30. A Hayati Configuration

The robot was formed into a mobile closed kinematic chain using a ball-bar mechanism. The ball-bar was attached to robot and the base plate in the same way as the DH configuration. It was modelled based on the DH-Hayati and the frames were allocated as shown in figure 31 . 


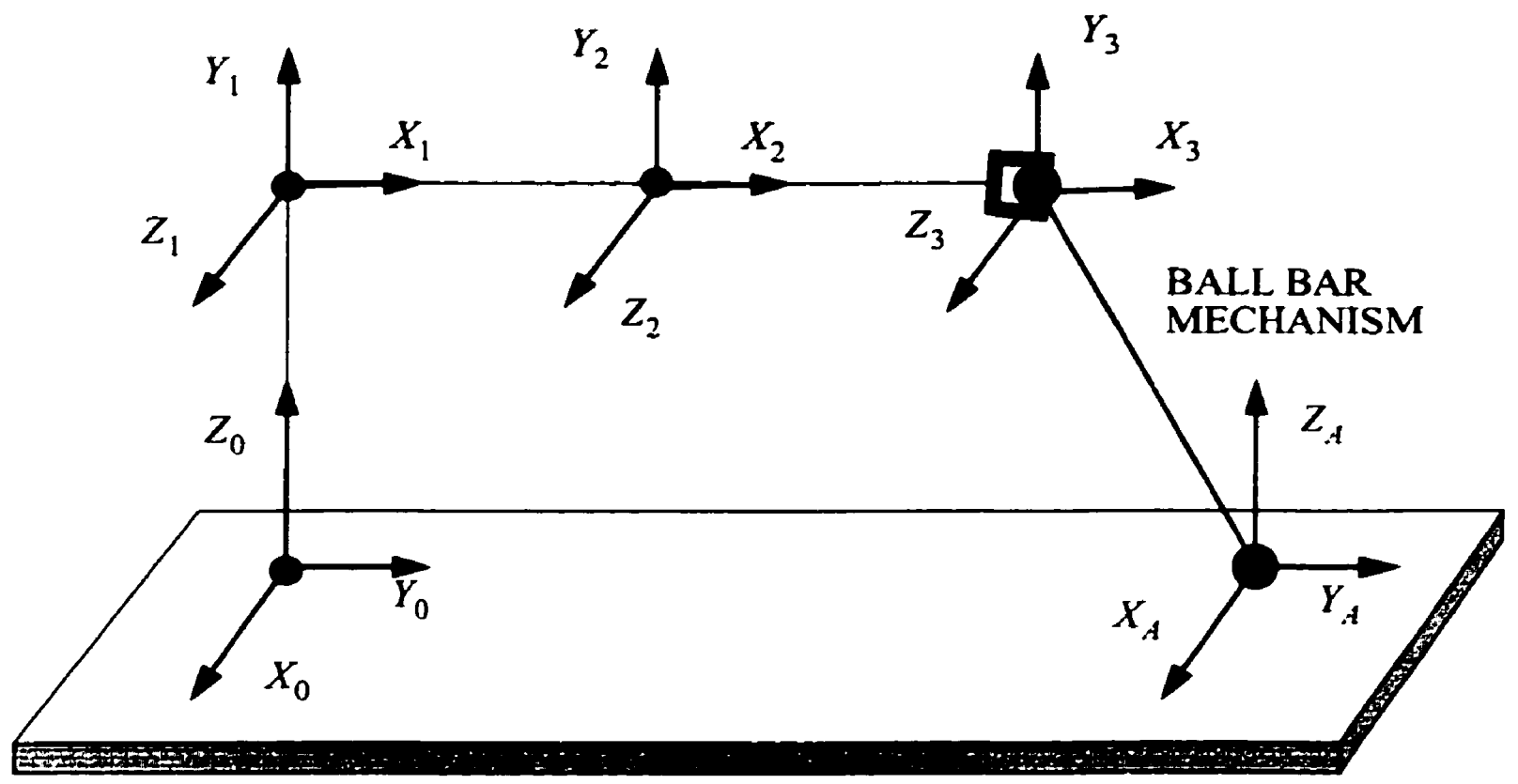

Figure 31. Frame Allocation for the Hayati Configuration

Nominal measurements were made on the robot based on the frame allocation scheme by marking points on the robot and taking measurements using a foot ruler. These measurements are reported in table 5 .

TABLE S. Nominal parameters for Hayati configuration

\begin{tabular}{c||ccccc}
\hline Link Number & $\begin{array}{c}\text { Length (l) in } \\
\mathrm{mm}\end{array}$ & $\begin{array}{c}\text { Link Offset ( } \mathrm{r}) \\
\text { in } \mathrm{mm}\end{array}$ & $\begin{array}{c}\text { Twist Angle } \alpha \\
\text { in degrees }\end{array}$ & $\begin{array}{c}\text { Hayati Angle } \beta \\
\text { in degrees }\end{array}$ & $\begin{array}{c}\text { Encoder offset } \\
\delta \theta \text { in degrees }\end{array}$ \\
\hline 1 & $l_{1}=0$ & $r_{1}=464.34$ & $\alpha_{1}=90^{\circ}$ & $\cdots$ & $\delta \theta_{1}=0^{\circ}$ \\
2 & $l_{2}=247.65$ & $\ldots$ & $\alpha_{2}=90^{\circ}$ & $\beta_{2}=0^{\circ}$ & $\delta \theta_{2}=0^{\circ}$ \\
3 & $l_{3}=246.528$ & $r_{3}=0$ & $\alpha_{3}=0^{\circ}$ & $\ldots$ & $\delta \theta_{3}=0^{\circ}$
\end{tabular}


The calibration user-interface was initiated and the nominal parameters specified. By moving the location of point $A$ around the base plate and varying the length of the bar mechanism, readings at 120 points in the entire workspace were taken. The process of collecting the points took about forty minutes. Parameters were identified using the data coilected. These values are reported in table 6.

TABLE 6. Identified Parameters

\begin{tabular}{|c|c|c|}
\hline & Nominal Value & Identified Value \\
\hline$l_{1}$ in $\mathrm{mm}$ & 0.0000 & 20.0712 \\
\hline$r_{1}$ in $\mathrm{mm}$ & 464.34 & 488.1385 \\
\hline$\alpha_{1}$ in degrees & 90.0000 & 89.5692 \\
\hline$\delta \theta_{1}$ in degrees & 0.0000 & -0.2131 \\
\hline$l_{2}$ in $\mathrm{mm}$ & 247.65 & 248.1366 \\
\hline$\beta_{2}$ in degrees & 0.0000 & -0.9629 \\
\hline$\alpha_{2}$ in degrees & 0.0000 & -0.4533 \\
\hline$\delta \theta_{2}$ in degrees & 0.0000 & -1.1825 \\
\hline$l_{3}$ in $\mathrm{mm}$ & 246.528 & 237.5159 \\
\hline$r_{3}$ in $\mathrm{mm}$ & 0.0000 & -4.044 \\
\hline$\alpha_{3}$ in degrees & 0.0000 & 0.0000 \\
\hline$\delta \theta_{3}$ in degrees & 0.0000 & -1.2608 \\
\hline
\end{tabular}


To check the success of calibration, the identified parameters were used to calculate the error in the bar length. The results are plotted in figure 32. Figure 33 shows a frequency distribution of the error in bar length. The mean and the standard deviation of the error in the bar length using nominal and identified values are shown in Table 7.

TABLE 7. Mean and SD

\begin{tabular}{l||cc}
\hline & $\begin{array}{c}\text { Using Nominal } \\
\text { parameters }\end{array}$ & $\begin{array}{c}\text { Using Identified } \\
\text { parameters }\end{array}$ \\
\hline \hline Mean error in bar length & $5.8884 \mathrm{~mm}$ & $0.0040 \mathrm{~mm}$ \\
$\begin{array}{c}\text { Standard deviation of the } \\
\text { error in bar length }\end{array}$ & $2.6845 \mathrm{~mm}$ & $1.4327 \mathrm{~mm}$ \\
\end{tabular}




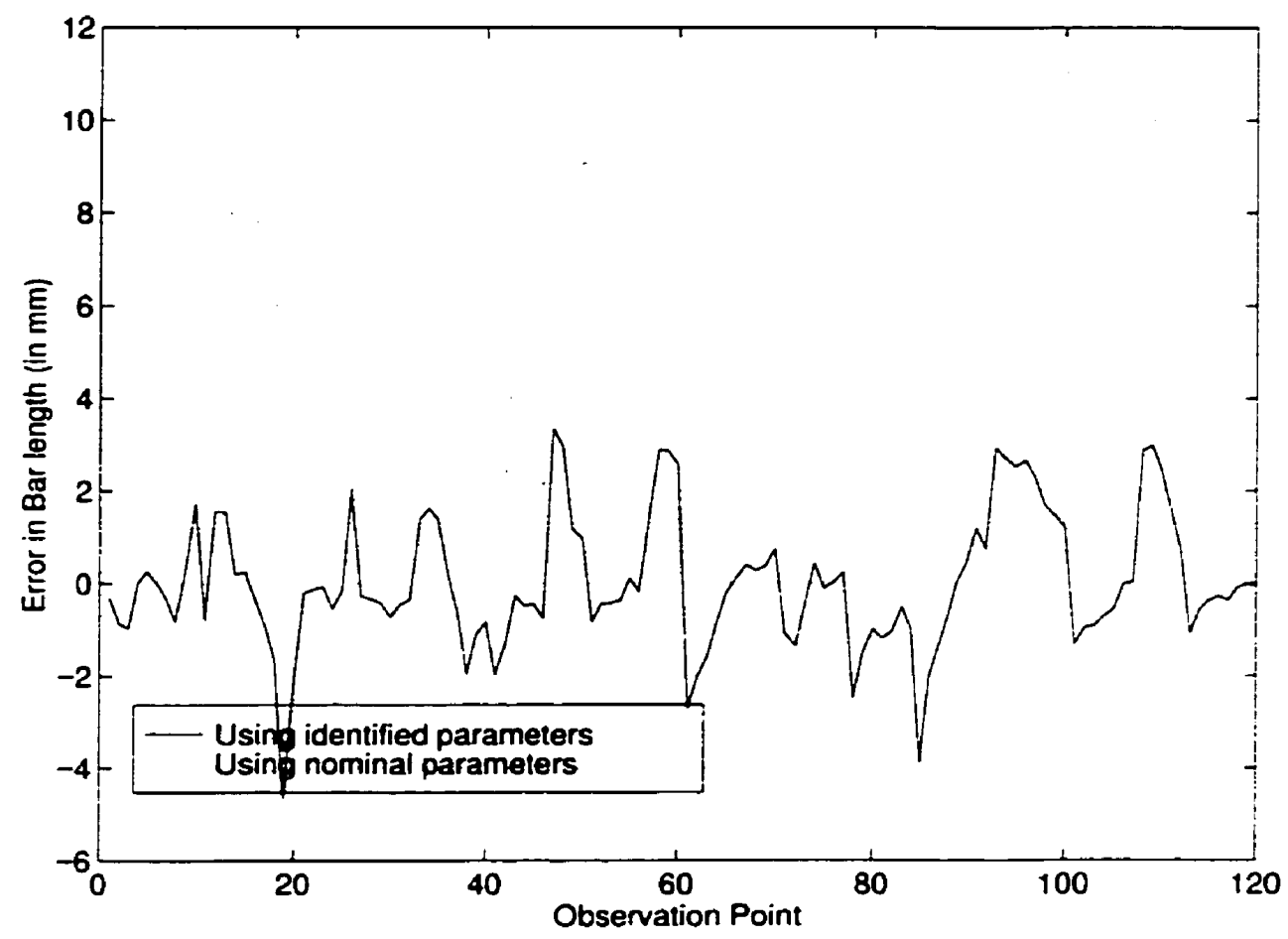

Figure 32. Error in Bar Length

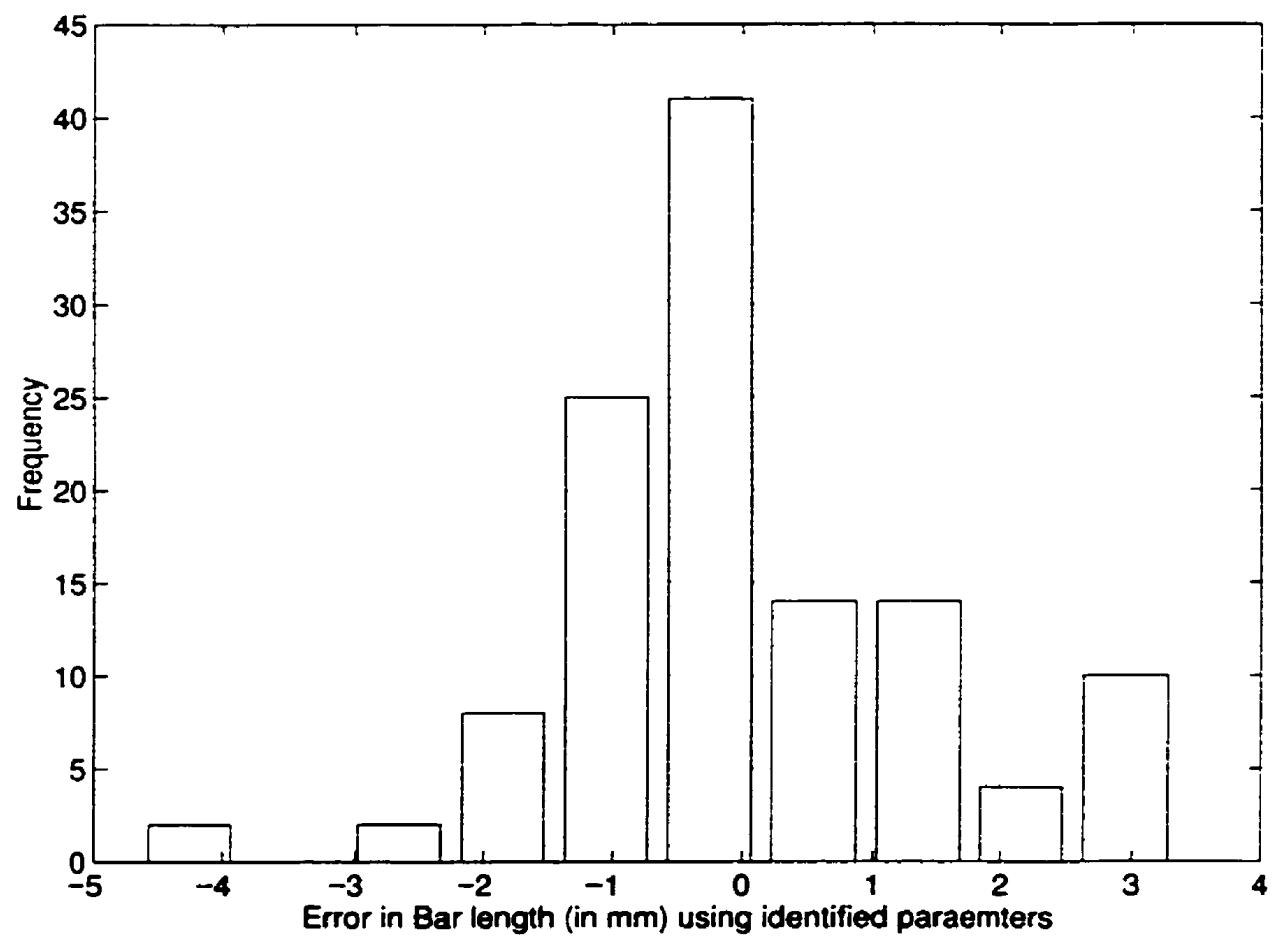

Figure 33. Frequency Distribution of Error in Bar Length 


\subsection{Analysis of Results}

A good measure of the accuracy of the system can be obtained by looking at the mean values of the error in the bar lengths at the observation points. In the DH configuration, the mean error was reduced from $10.027 \mathrm{~mm}$ to less than $-0.06 \mathrm{~mm}$ (tables 3 and 4 ). In the Hayati configuration, the mean error was reduced from $5.8884 \mathrm{~mm}$ to $0.004 \mathrm{~mm}$ (table 7). This showed an improvement in accuracy of at least $90 \%$.

Referring to tables 3,4 and 7, the standard deviations of the error distributions was reduced from $3.2812 \mathrm{~mm}$ to less than $1.39 \mathrm{~mm}$ for the $\mathrm{DH}$ configuration and from $2.6845 \mathrm{~mm}$ to 1.4327 $\mathrm{mm}$ for the Hayati configuration. The relatively high standard deviations can be attributed to mechanical problems with RoboTwin joints. A 'backlash effect' was observed in the RoboTwin joints due to the wear of the mechanical connection between the motor and the gear train. As the encoders are attached after the gear train, the readings were not very reliable. This effect was observed only in the second joint. However, realizing that the wear was due to a design defect in the joints, the 'backlash effect' could be playing a part in all the joints.

While calibrating the DH configuration, four different data sets were used. The parameters identified are shown in table 2 . The low range of variation in the identified parameters indicate that 99 randomly chosen produced results as good as 1440 points chosen over the entire work space. 


\section{Chapter 5}

\section{Conclusions and Future Work}

A methodology for calibrating the RoboTwin was developed. Calibration was achieved by modelling the robot configuration, taking measurements on the robot and identifying parameters representing the robot. The methodology developed is independent of the number of joints in the chosen RoboTwin configuration.

The DH-Hayati model was used to model the RoboTwin. The robot configurations were modelled based on the Hayati convention for nominally parallel joints and based on the DH convention otherwise.

A new passive measurement scheme was developed to collect partial pose data of the robot. A 6-dof ball-bar mechanism with a known bar length was used as a measurement device. The ball-bar mechanism consisted to of two 3-dof ball joints attached to the two ends of the bar. A closed kinematic chain was formed using the ball-bar mechanism by screwing one ball joint in the base plate and holding the other joint with the robot's gripper. This closed kinematic chain was moved in the workspace and joint angle data was collected at different points in the workspace. bars of different lengths were used and the ball bar mechanism was screwed at different points on the base plate, to increase the workspace covered.

Using nominal values of the robot parameters and the joint angle data collected at different observation points, the nominal length of the bar was calculated. The error in the bar was 
calculated using the nominal length and the known exact length. The error in the bar length was minimized using a linear least squares algorithm iteratively, to determine the correct robot parameters.

A new method of calculating the Jacobian matrix representing a change in the bar length with respect to the robot parameters was developed. This made it possible to use a linear least squares method iteratively and achieve a faster convergence.

A user friendly calibration interface was developed to simplify the task of taking measurements and performing identification. The user is led through the task of calibration by means of several screens. The interface was programmed as a separate module in the existing RoboTwin user-interface.

The calibration methodology developed was implemented on two RoboTwin configuration. At least $90 \%$ improvement in the accuracy was observed. The calibration methodology implemented met the initial design criterion and was able to accommodate reconfigurability, modularity and expandability. It is inexpensive and easy to use.

This methodology can be used for calibrating any robot where the motors could be moved manually with power removed.

\subsection{Future Work}

Better accuracy of the RoboTwin can be achieved by improving the joint design and calibrating the robot using the calibration methodology developed. A 'backlash effect' in the RoboTwin joints was observed due to the improper fitting of the motor and the gear train making the joint encoder readings unreliable. This effect was observed in joints that were used 
extensively and became more prominent due to the wear in the pins connecting the motor and the gears.

The calibration system developed provides the user with the corrected robot parameters. The user is expected to use these parameters to obtain an inverse kinematics solution and implement this in the controller. To automate the process of implementing the results, a subroutine could be written to use the results of calibration and update the controller. 


\section{References}

[1] Renders J., Rossingnol E., Becquet M., Hanus R., "Kinematic calibration and geometrical parameter identification for robots". IEEE Transactions on Robotics and Automation, 1991, v.7 no.6, pp. 721-732.

[2] Roth Z., Mooring B.W., Ravani B., "An overview of robot calibration", IEEE Journal of Robotics and Automation, 1987, RA-3, pp. 331-340.

[3] Shamma J. S., Whitney D. E., "A method for inverse robot calibration", Journal of Dynamic Systems, Measurement, and Control, 1987, v.109 no.1, pp. 36-43.

[4] Denavit J., Hartenberg R.S., "A kinematic notation for lower pair mechanisms based on matrices". Journal of Applied Mechanics, 1955, v.22, pp. 215-221.

[5] Paul, R. P., Robot Manipulators: Mathematics, Programming and Control, The MIT Press, 1982.

[6] Kirchner H.O.K., Gurumoorthy B., Prinz F.B., "A pertubation approach to robot calibration", International Journal of Robotics Research, 1987, v.6 no.4, pp. 47-59. 
[7] Schroer K., "Theory of kinematic modelling and numerical procedures for robot calibration", Robot Calibration, ed. Bernhardt R and Albright S., 1993, pp. 157-193.

[8] Ibarra R., Perreira N. D., "Determination of linkage parameter and pair variable errors in open chain kinematic linkages using a minimal set of pose measurement data", Journal of Mechanisms, Transmissions, and Automation in Design, 1986, pp. 159-166.

[9] Hayati S. A., Mirmirani M., "Improving the absolute positioning accuracy of robot manipulators", Journal of Robotics Systems, 1985, v.2, pp 397-413.

[10] Judd R. P., Knasinski A. B., “A technique to calibrate industrial robots with experimental verification", Proceedings of IEEE International Conference of Robotics and Automation. 1987, pp. 351-357.

[11] Sugimoto K., Okada T., "Compensation of positioning errors caused by geometric deviations in the robot system", Robotics Research: The Second International Symposium, 1985, pp. 231-236.

[12] Vietschegger W. K., Wu C-H., "Robot accuracy analysis based on kinematics", IEEE Journal of Robotics and Automation, 1986, RA-2, pp. 171-179.

[13] Everett L., Suryohadiprojo A., "A study of kinematic models for forward calibration of manipulators", Proceedings of IEEE International Conference on Robotics and Automation, 1988, pp. 798-800.

[14] Chen J., Chao L. M., "Positioning error analysis for robot manipulators with all rotary joints", Proceedings of IEEE International Conference on Robotics and Automation, 1986, pp. 1011-1016.

[15] Sheth P. N., Uicker J. J., "A generalized symbolic notation for mechanisms", $A S M E$ Journal of Engineering for Industry, 1971, v.93 no.1, pp 102-112, 1971. 
[16] Ziegert J., Datseris P., "Simplified Method for kinematic calibration of robots", Proceedings of ASME International Manufacturing Conference, 1990, pp. 177-185.

[17] Goswami A., Quaid A., Peshkin M., “Identifying robot parameters using partial pose information", IEEE Control Systems, October 1993, pp. 6-14.

[18] Everett L. J., Driels M., Mooring B. W., "Kinematic Modelling for Robot Calibration", Proceedings of 1987 IEEE International Conference of Robotics and Automation, 1987, pp. 183-189.

[19] Everett L. J., Wong H. T., "Theory of Kinematic Parameter Identification for Industrial Robots", Journal of Dynamic Systems, Measurement and Control, 1988, v.10, pp. 96100.

[20] Ziegert J., Datseris P., "Basic Considerations for Robot Calibration", International Journal of Robotics and Automation, 1988, v.4, pp. 158-166.

[21] Whitney D. E., Lozinski C. A., Rourke J. M., "Industrial robot forward calibration method and results", Journal of Dynamic Systems, Measurement, and control, 1986, v. 108 no. 1 , pp. $1-8$.

[22] Lau K., Hocken R. J., Haight W. C., “Automatic laser tracking interferometer system for robot metrology", Precision Engineering, 1986, v.8 no.1.

[23] Stone H. W., Sanderson A. C., "A prototype arm signature identification system", Proceedings of IEEE International Conference on Robotics and Automation, 1987, pp. 175-182.

[24] Stone H. W., Sanderson A.C., Neuman C. P., “Arm signature identification", Proceedings of IEEE International Conference on Robotics and Automation, 1986, pp. 41-48. 
[25] Hollerbach J. M., Bennett D. J., "Automatic kinematic calibration using a motion tracking system", Robotics Research 4rth International Symposium. 1988, pp. 191-198.

[26] An C. H., Atkeson C. G., Hollerbach J. M., Model Based Control of a Robot Manipulator, 1988, pp. 49-64.

[27] Vietschegger W. K., Wu C-H., "A method for calibrating and compensating robot kinematic errors", Proceedings of IEEE International Conference on Robotics and Automation, 1987, pp. 39-44.

[28] Borm J-H., Menq C-H., "Experimental study of observability of parameters errors in robot calibration", Proceedings of IEEE Conference on Robotics and Automation, 1989, pp. 587-592.

[29] Tang G-R, Liu L-S, “A study of three robot calibration methods based on flat surfaces", Mechanisms and Machine Theory, 1994, v.29, pp. 195-206.

[30] Bennett D. J., Hollerbach J. M., "Self calibration of single loop, closed kinematic chains formed by dual or redundant manipulators." Proceedings of 27th IEEE Conference on Decision and Control, 1988, pp. 627-629.

[31] Bennett D. J., Hollerbach J. M., "Autonomous calibration of single loop closed kinematic chains formed by manipulators with passive end point constraints", IEEE Transaction on Robotics and Automation, 1991, v.7 no.5, pp. 597-606.

[32] Bennett D. J., Hollerbach J. M., "Identifying the kinematics of robots and their tasks", Proceedings of IEEE Conference on Robotics and Automation, 1988, pp. 580-586.

[33] Bennett D. J., Hollerbach J. M., “Autonomous robot calibration for hand-eye coordination", International journal of Robotics Research, 1991, v.10 no.5, pp. 550-559. 
[34] Hollerbach J. M., “Closed loop kinematic calibration of the RSI 6-dof hand controller”, Proceedings of IEEE International Conference on Robotics and Automation, 1993, pp. 142-148.

[35] Bennett D. J., Hollerbach J. M., “Closed loop kinematic calibration of the Utah-MIT Hand", Experimental Robotics I - The First International Symposium. 1989, pp. 539553.

[36] Driels M. R., "Using passive end-point motion constraints to calibrate robot manipulators", Journal of Dynamic Systems, Measurement and Control, 1993, v.115 no.3, pp. 560-566.

[37] Stone H. W., Kinematic Modelling, Identification, and Control of Robotic Manipulators, Kluwer Academic Publishers, Boston 1987.

[38] Sklar M., "Geometric calibration of industrial manipulators by circle point analysis", Proceedings of Second Conference on Recent Advances in Robotics, 1987, pp. 175-182.

[39] Driels M., Pathre U., "Significance of observation strategy on design of robot calibration experiments", Journal of Robotic Systems, 1990, v.7, pp. 197-223.

[40] Driels M., Pathre U., "Simulation experiments on parameter identification for Robot Calibration", International Journal of Advanced Manufacturing Technology, 1990. v.5, pp. 13-33.

[41] Ananthanarayanan S. P., Szymczyk C., Goldenberg A.A., "Identification of kinematic parameters of multiple closed chain robot manipulators working in coordination", Proceedings of IEEE International Conference on Robotics and Automation, 1992, v.1 pp. 358-363. 
[42] Broderick P. L., Cipra R. J., "A method for determining and correcting robot position and orientation errors due to manufacturing", Journal of Mechanical Transmissions and Automation in Design, v.110, n.1.pp. 3-10.

[43] Hui R., Kircanski N., Goldenberg A., Zhou C., et al, "Design of the IRIS Facility - A Modular, Reconfigurable and Expandable Robot Test Bed", Proceedings of IEEE International Conference on Robotics and Automation, 1992, pp. 155-160.

[44] Hui R., Detailed Design Report for the IRIS Facility, RAL Internal Report, Department of Mechanical Engineering, University of Toronto, Toronto, Canada, 1992.

[45] Kircanski N. M., IRIS Grasping and Manipulation Facility: Performance Evaluation, Modelling and Parameter Identification, RAL Internal Report, Department of Mechanical Engineering, University of Toronto, Toronto, Canada, 1993. 


\section{Appendix : Program Listing}

The program developed for the user interface is presented here. This does not depend or interfere with the other robot control programs.

pragma off (Align_members);

\#define inport(port) inpw(port)

\#define outport(port, v) outpw(port, v)

\# define pi 3.141593

\# include <dos.h>

\# include <stdio.h>

\# include <conio.h>

\# include <ctype.h>

\# include <signal.h>

\# include <io.h>

\# include <graph.h>

\# include <string.h>

\# include <stdlib.h>

\# include <math.h>

\# include <fontl.h>

\# include <sysltypes.h>

\# include <syslstat.h> 
\# include "iris_lib.c"

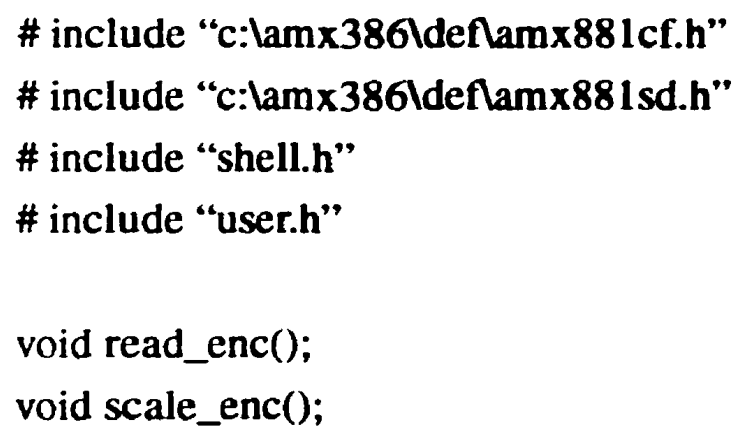

float minor( float $\mathbf{A}[3][3]$, int row, int col);

float minor3 ( float $A[4][4]$, int row, int col);

void adj ( float $B[8][200]$, float $C[200][8]$ );

void inverse(float $A[4][4]$, float $D[4][4])$;

void multiply ( float $A[4][4]$, float $D[4][4]$,float $E[4][4]$ );

void cross(float yi[3], float bil[3], float Jbi[3]);

int config_rep[8]; 
float len[8], off[8], alp[8] ,bet[8], the[8];

float dlen[8], doff[8], dalp[8] ,dbet[8], dthe[8];

void store_encoder(void);

void change_barl(void);

void change_base(void);

void scr_frame(void);

void update_table(void);

void show_enc(void);

char lst[20],njts[10],warm[10],buffer[200];

float paramts[8][4],enc_data[200][12];

int $i, j$, no_of_joints, which_joints[8], which_arm;

int nobs $[10]=\{0\}$,bar_ptr $=0$,lbar_flag $=0$,tobs $=0$;

float $\operatorname{lbar}[10]=\{0\}$;

float Ix,ly,lz;

STATES st 1;

INP_SIGNALS sg 1;

void specify_config(void)

l

char c;

for $(\mathrm{i}=0 ; \mathrm{i}<8 ; \mathrm{i}++)$

which_joints $[\mathrm{i}]=0$;

calibration_scr_10);

$\mathrm{c}=\operatorname{getch}()$;

while (c!=RETURN \&\& c!=ESCAPE)

$c=\operatorname{getch}()$;

if ( $c=$ ESCAPE)

return;

calibration_scr_2();

sloop: calibration_scr_3();

calibration_scr_4();

$c=\operatorname{getch}()$; 


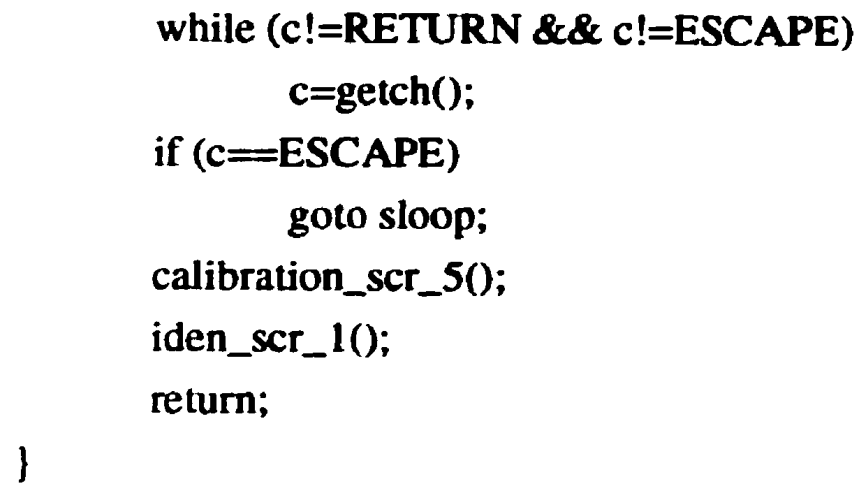


else

no_of_joints $=$ njts $[0]-48$;

if (no_of_joints>8 || no_of_joints <1)

goto loopa;

loopb:_settextposition (14,3);

_outtext("Which arm are you working with (1 or 2) (Default 2)");

fflush(stdin);

gets(warm);

if (warm[0]='10')

which_arm=2;

else

which_arm = warm $[0]-48$;

if (which_arm! $!$ \&\&which_arm $!=2$ )

goto loopb;

_outtext("In Specify the joints you are working with.");

_outtext("In This can be learn by studying the amplifier connections");

_outtext("In Enter the joints as a list separated by commas.");

_outtext("In Terminate the list by a retum (Default 2,3,4):");

fflush(stdin);

gets(lst);

$\mathrm{i}=0$;

$\mathrm{j}=0$;

if (lst[0] $\left.==^{\prime} 10^{\prime}\right)$

I

which_joints $[0]=2$;

which_joints $[1]=3$;

which_joints $[2]=4$;

\}

while (lst[i] !=’10')

1

$$
\begin{aligned}
& \text { if }(\operatorname{lst}[\mathrm{i}]==, ?) \\
& \text { it+; }
\end{aligned}
$$




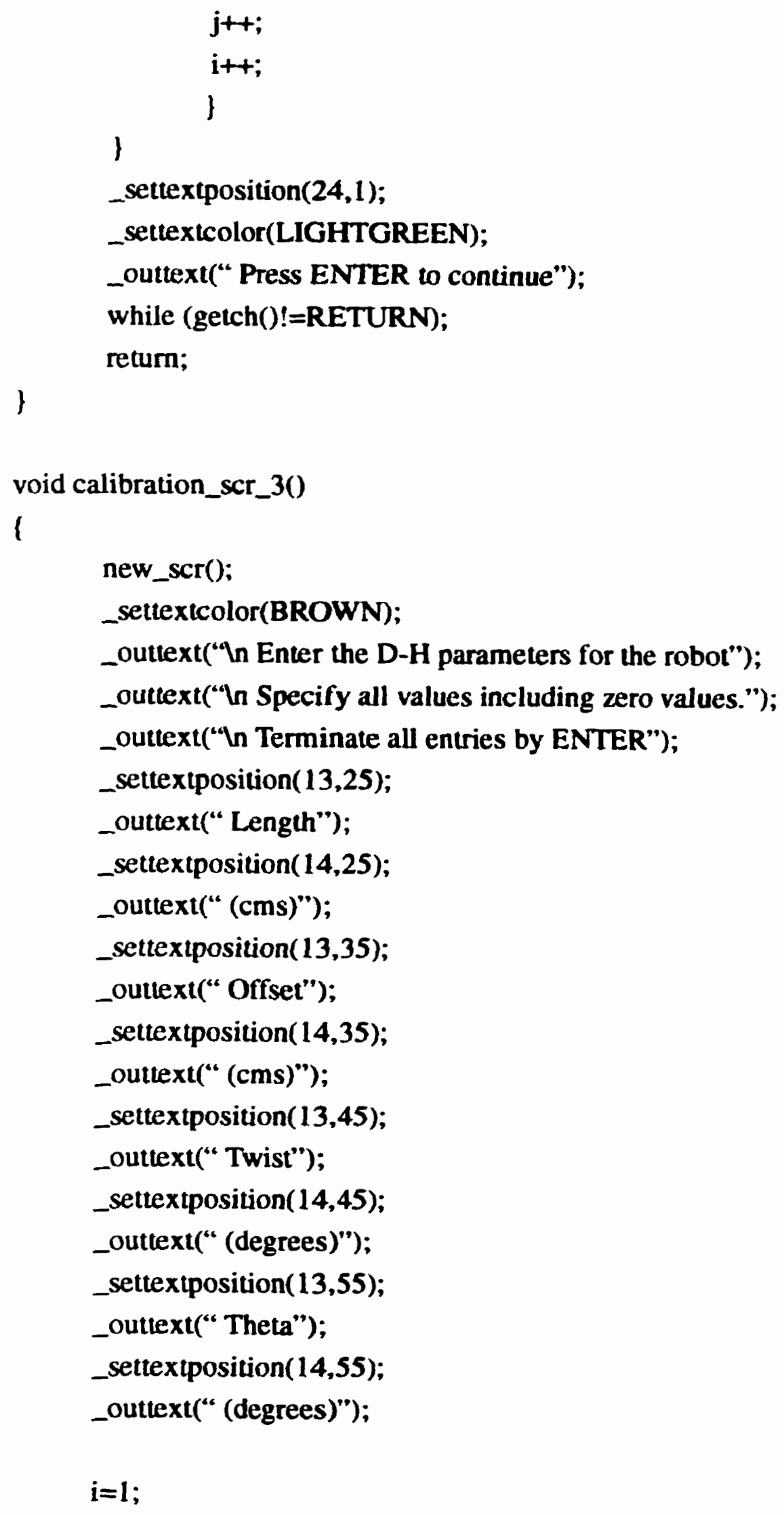




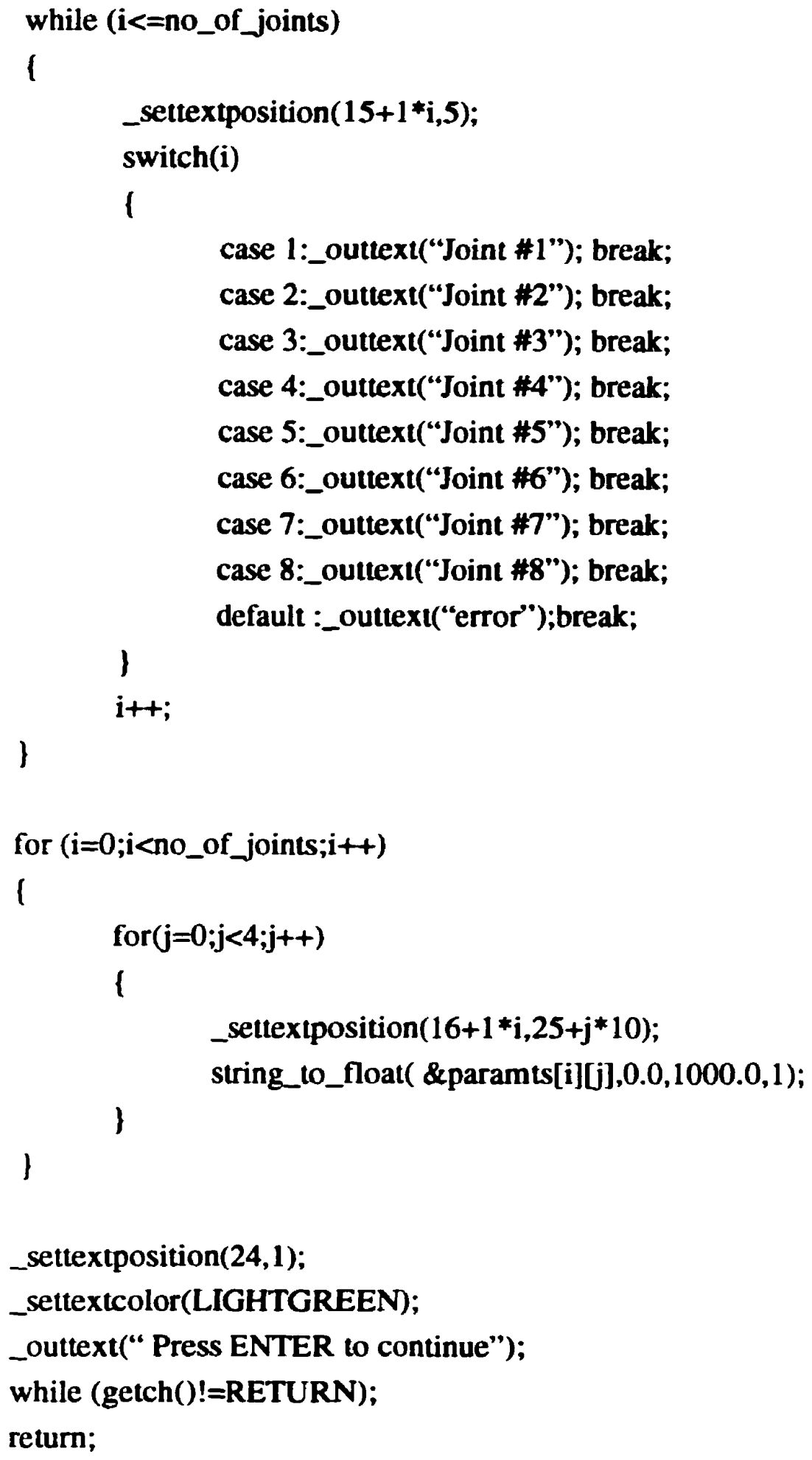


new_scr();

_settextcolor(BROWN);

_outtext( 'In Parameters entered by you are :");

_settextposition( $(1,25)$;

_outtext(" Length");

_settextposition(12,25);

_outtext(" (cms)");

_settextposition $(11,35)$;

_outtext(" Offset");

_settextposition(12,35);

_outtext(" (cms)");

_settextposition $(11,45)$;

_outtext(" Twist");

_settextposition(12,45);

_outtext(" (degrees)");

_settextposition(11,55);

_outtext(" Theta");

_settextposition(12,55);

_outtext(“" (degrees)");

/*_settextposition(12,25);

_outtext(" Length (cms)");

_settextposition(12,40);

_outtext(" Offset (cms)");

_settextposition(12,55);

_outtext(“ Twist (degrees)"); *I

$\mathrm{i}=1$;

while (i<=no_of_joints)

I

_settextposition $(13+1 * i, 5)$;

switch(i)

I

case 1:_outtext(“Joint \#1"); break; 


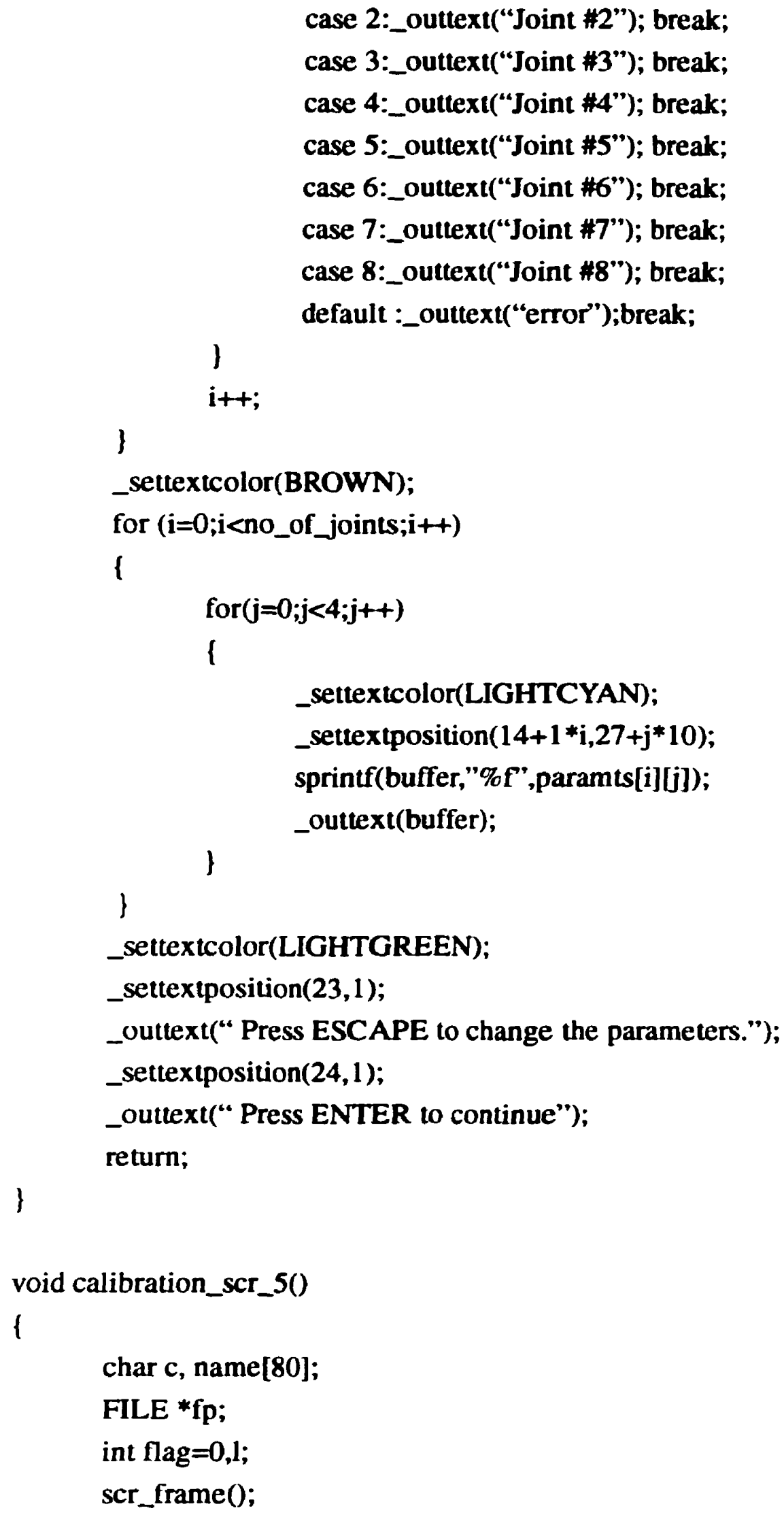




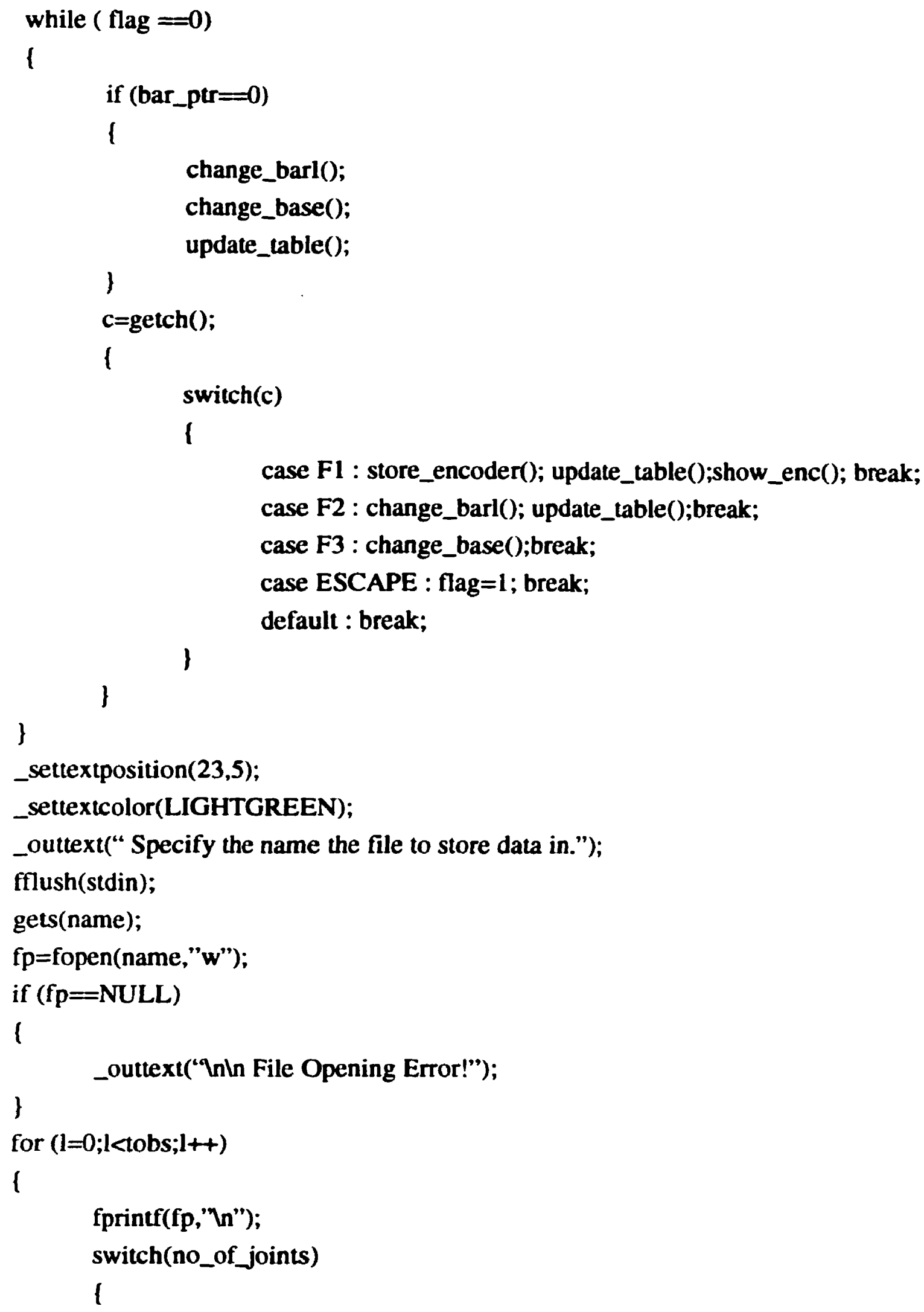




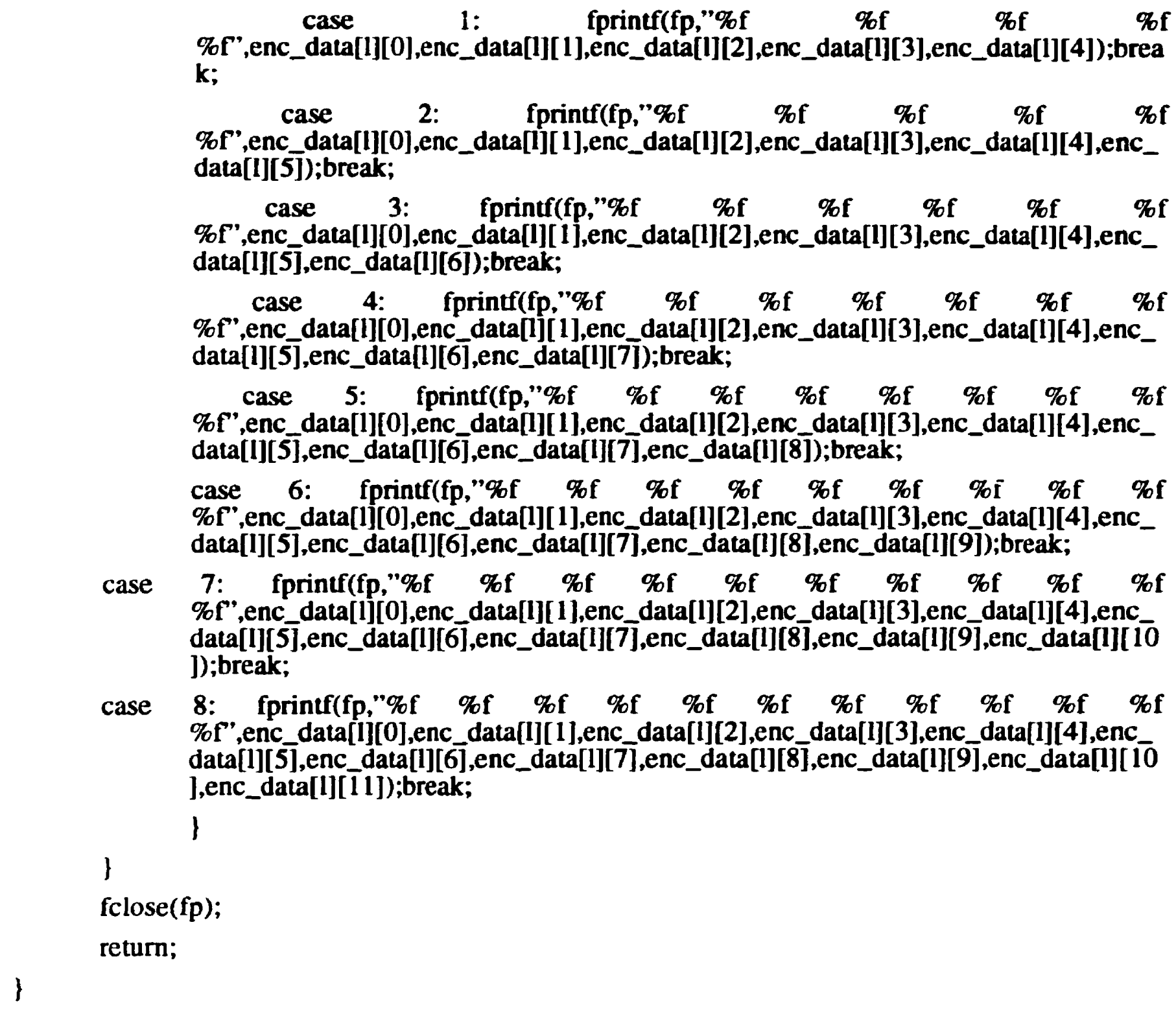
\%f",enc_data[1][0],enc_data[1][1],enc_data[1][2],enc_data[1][3],enc_data[1][4],enc_ data[l] [5], enc_data[1][6], enc_data[1][7],enc_data[1][8]); break;

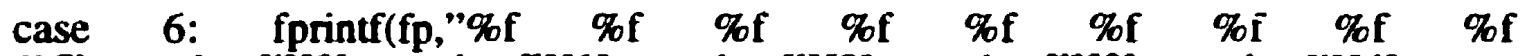
\%f",enc_data[1][0],enc_data[1][1],enc_data[1][2],enc_data[1][3],enc_data[1][4],enc_ data[1][5], enc_data[1][6], enc_data[1][7] ,enc_data[1][8],enc_data[1][9]); break;

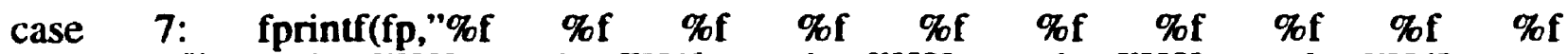
$\% \mathrm{f}^{\prime}$, enc_data[1][0],enc_data[1][1],enc_data[1][2],enc_data[1][3],enc_data[1][4],enc data[1][5],enc_data[1]][6],enc_data[1][7], enc_data[1][8],enc_data[1]][9],enc_data[1][10 ]);break;

case 8: fprintf(fp,"\%f $\% \mathrm{f} \quad \% \mathrm{f} \quad \% \mathrm{f} \quad \% \mathrm{f} \quad \% \mathrm{f} \quad \% \mathrm{f} \quad \% \mathrm{f} \quad \% \mathrm{f} \quad \% \mathrm{f}$ $\%$ f",enc_data[1][0],enc_data[I][1],enc_data[1][2],enc_data[1][3],enc_data[1][4],enc

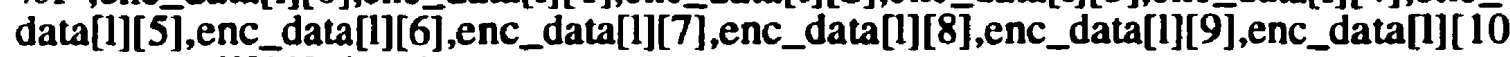
],enc_data[1][11]);break;

\}

l

fclose(fp);

return;

void iden_scr_1(void)

1

new_scr();

// Clear the screen and confirm that the encoders are initilized

_settextcolor(BROWN);

_settextposition(15,5);

_outtext(“Do you want to perform identification ?");

_settextposition(17,5);

_outtext("Press F1 to say YES or press ESCAPE to say NO"); 


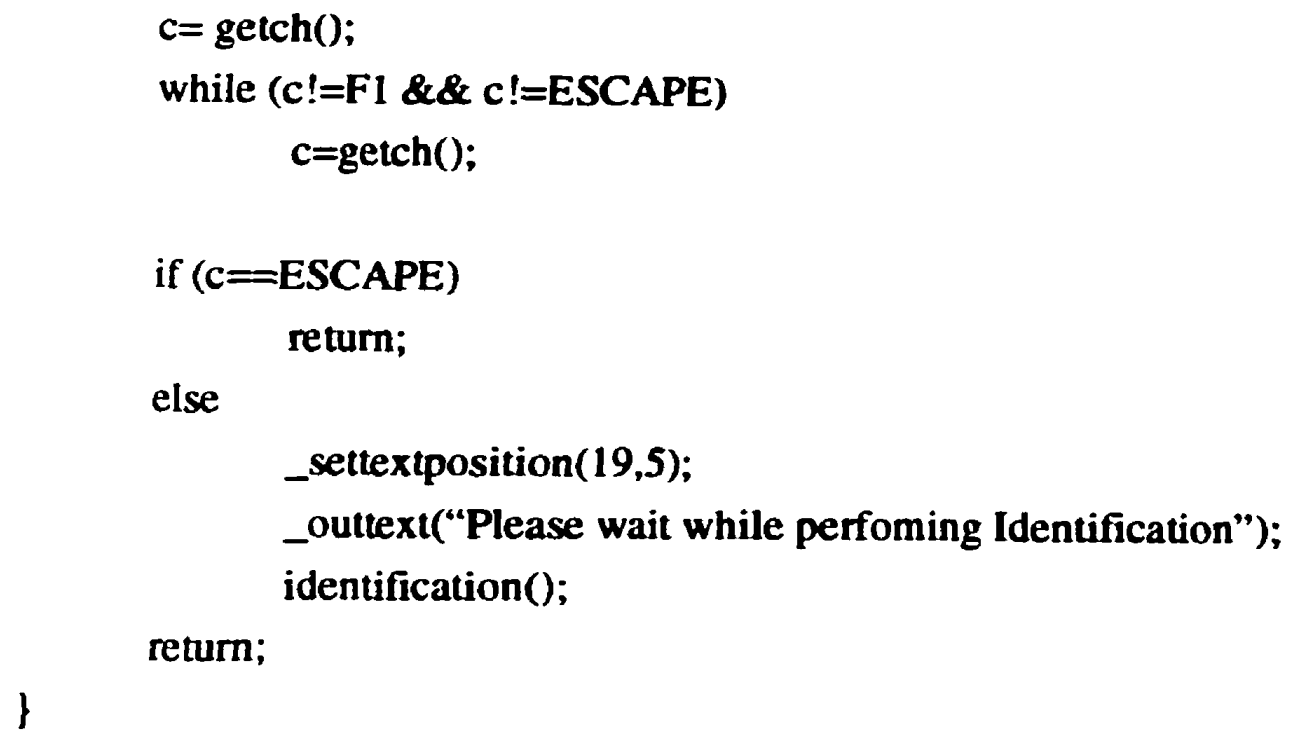

int $1, f$;

float enc_d[8];

$\mathrm{f}=0$;

read_enc();

scale_enc(); 


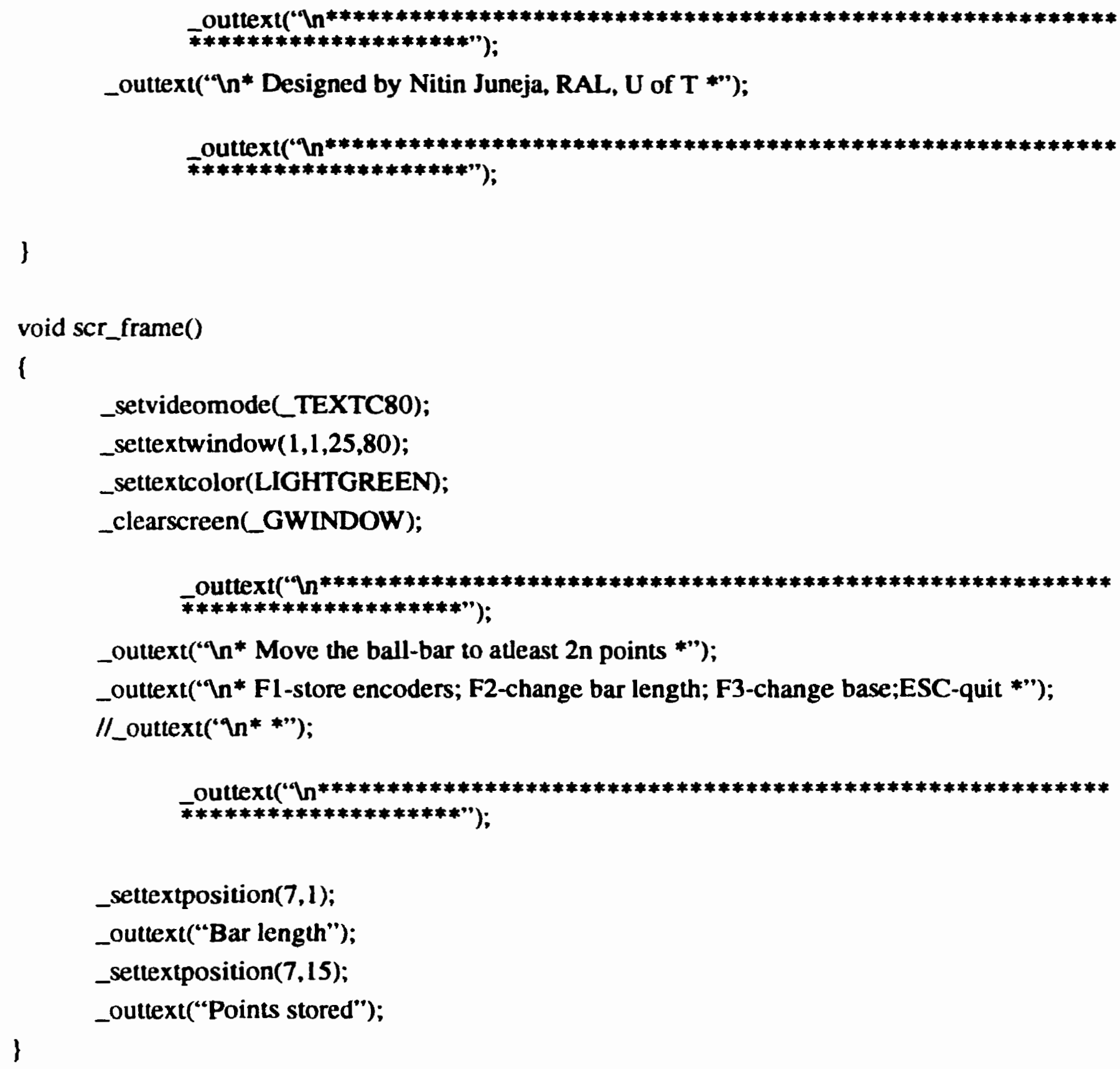




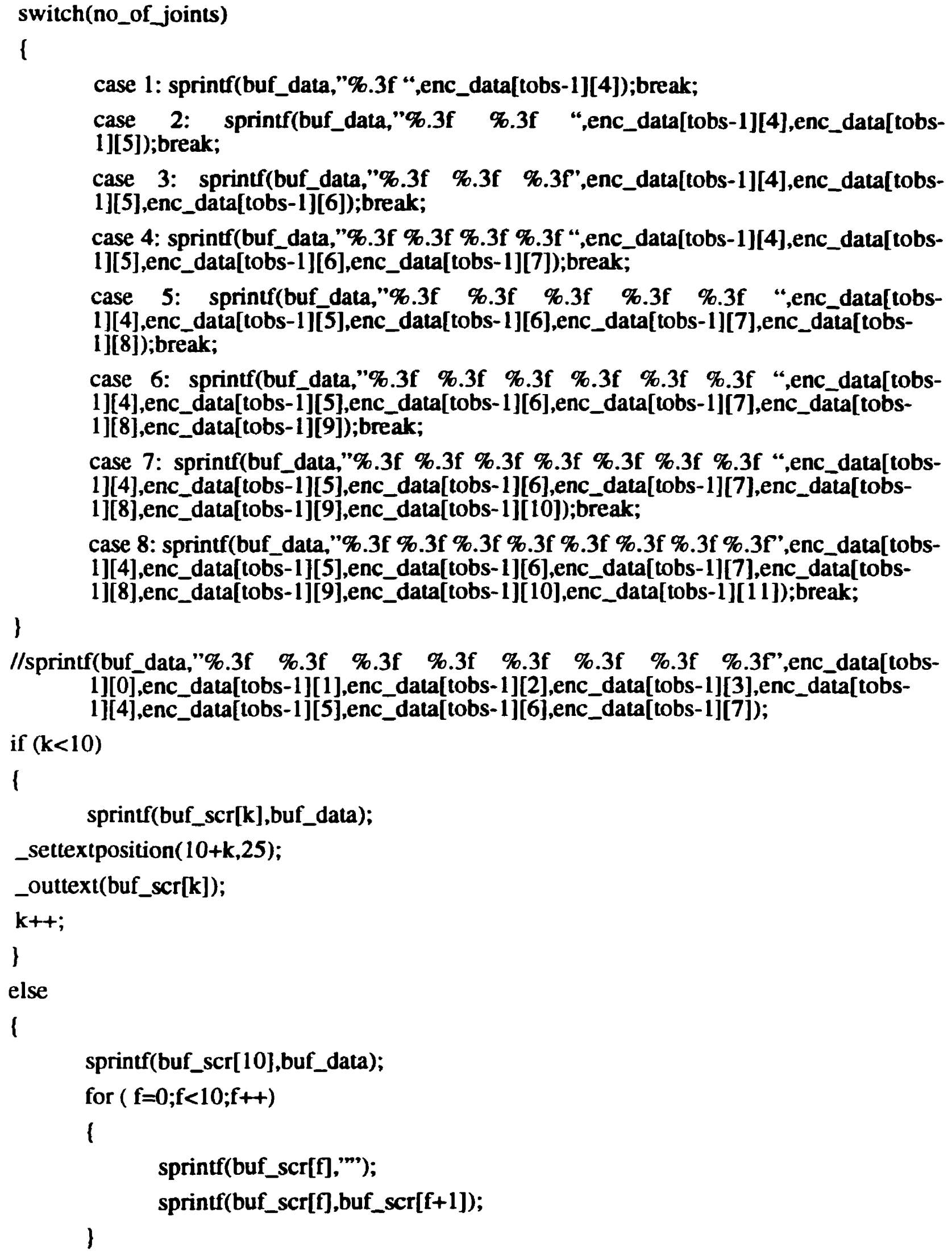

case 1: sprintf(buf_data,"\%.3f ",enc_data[tobs-1][4]);break;

case 2: sprintf(buf_data,"\%.3f \%.3f "enc_data[tobs-1][4],enc_data[tobs1][5]);break;

case 3: sprintf(buf_data,"\%.3f \%.3f \%.3f",enc_data[tobs-1][4],enc_data[tobs1][5],enc_data[tobs-1][6]); break;

case 4: sprintf(buf_data,"\%.3f \%.3f \%.3f \%.3f ",enc_data[tobs-1][4],enc_data[tobs1][5],enc_data[tobs-1][6],enc_data[tobs-1][7]);break;

case 5: sprintf(buf_data,"\%.3f \%.3f \%.3f \%.3f \%.3f “,enc_data[tobs1][4],enc_data[tobs-1][5],enc_data[tobs-1][6],enc_data[tobs-1][7],enc_data[tobs1][8]);break;

case 6: sprintf(buf_data,"\%.3f \%.3f \%.3f \%.3f \%.3f \%.3f “,enc_data[tobs1][4],enc_data[tobs-1][5],enc_data[tobs-1][6],enc_data[tobs-1][7],enc_data[tobs1][8],enc_data[tobs-1][9]); break;

case 7: sprintf(buf_data,"\%.3f \%.3f \%.3f \%.3f \%.3f \%.3f \%.3f “,enc_data[tobs1][4],enc_data[tobs-1][5],enc_data[tobs-1][6],enc_data[tobs-1][7],enc_data[tobs1][8],enc_data[tobs-1][9],enc_data[tobs-1][10]);break;

case 8: sprintf(buf_data,"\%.3f \%.3f \%.3f \%.3f \%.3f \%.3f \%.3f \%.3f",enc_data[tobs1][4],enc_data[tobs-1][5],enc_data[tobs-1][6],enc_data[tobs-1][7],enc_data[tobs1][8],enc_data[tobs-1][9],enc_data[tobs-1][10],enc_data[tobs-1][11]); break;

\}

//sprintf(buf_data,"\%.3f $\% .3 f \quad \% .3 f \quad \% .3 f \quad \% .3 f \quad \% .3 f \quad \% .3 f \quad \% .3 f$,enc_data[tobs1][0],enc_data[tobs-1][1],enc_data[tobs-1][2],enc_data[tobs-1][3],enc_data[tobs1][4],enc_data[tobs-1][5],enc_data[tobs-1][6],enc_data[tobs-1][7]);

if $(k<10)$

1

sprintf(buf_scr[k],buf_data);

_settextposition $(10+k, 25)$;

_outtext(buf_scr[k]);

$k++$;

\}

else

sprintf(buf_scr[10],buf_data);

for $(f=0 ; f<10 ; f++)$

1

sprintf(buf_scr[f],"');

sprintf(buf_scr[f],buf_scr[f+1]);

\} 


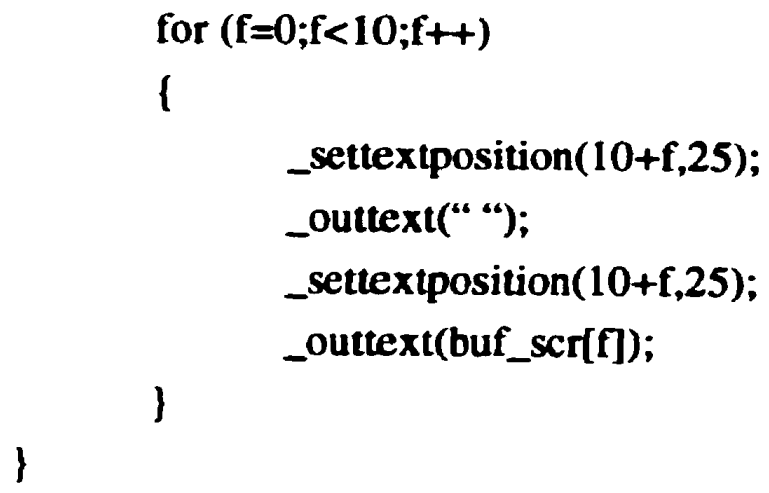




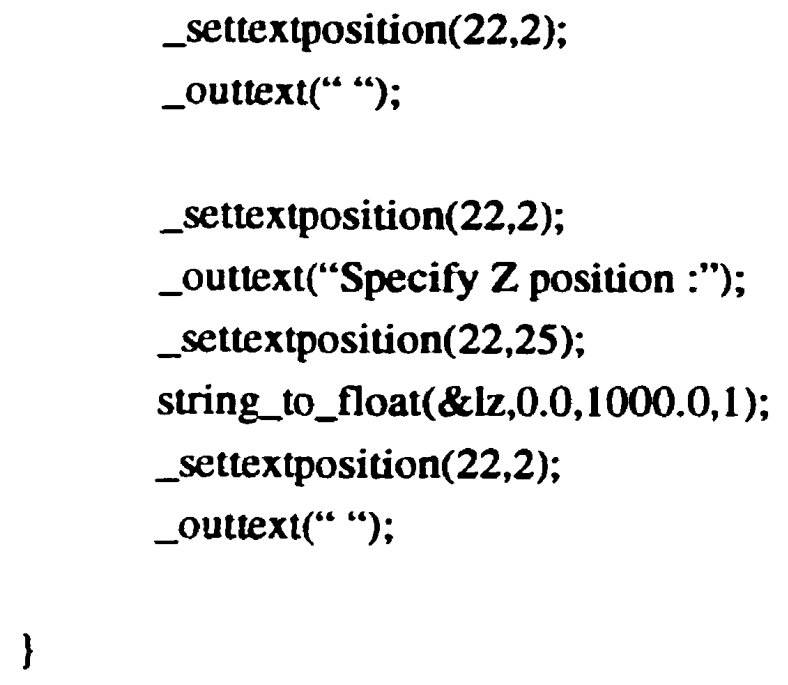


sg 1.arm2.iq[J4] = (int) pos_aux[8];

)
// Encoder: 500 CPR * 4
// Gear ratio: 51 for
$/ /$ joints $(3 a, 3 b$ and $4 a, 4 b)$
// Gear ratio: 101 for
// joints (1a,1b and 2a,2b)
// Encoder constant:
// 360 / number_of_pulses
// $360 /(500 * 4 *$ Gear_ratio)

\# define ECONV_J1 0.00178218 // Encoder constant: 360/202000

\# define ECONV_J2 0.00178218 // Encoder constant: 360/202000

\# define ECONV_J3 0.00352941 // Encoder constant: 360/102000

\# define ECONV_J4 0.00352941 // Encoder constant: 360/102000

void scale_enc() // This routine should go to screen task

\{

stl.arm 1.q[J1] = ECONV_Jl * (float) sgl $\operatorname{arm} 1 . \mathrm{iq}[\mathrm{J} 1]+36.18$;

st 1.arm 1.q[J2] = ECONV_J2 * (float) sg 1.arm1.iq[J2] + 11.05;

st l.arm 1.q[J3] = -ECONV_J3 * (float) sg1.arm 1.iq[J3] - 4.24 ;

st l.arm 1.q[J4] = ECONV_J4 * (float) sgl.arm l.iq[J4] + 0.0;

st l.arm2.q[J1] = ECONV_J1 * (float) sg1 .arm2.iq[J1] - 38.59489 1;

st 1.arm2.q[J2] = ECONV_J2 * (float) sg1.arm2.iq[J2] + 48.101036;

st1.arm2.q[J3] = ECONV_J3 * (float) sgl .arm2.iq[J3] + 0.0;

st 1.arm2.q[J4] = ECONV_J4 * (float) sg1.arm2.iq[J4] + 0.0;

了

void identification (void)

l

// Declare variables

int $\mathbf{u}, \mathbf{v}, \mathbf{w}$;

float theta[8];

float $K[8]=\{99,99,99,99,99,99,99,99\}$; 
float $M[200][8], D[200]$;

float $\mathrm{Mt}[8][200]$;

float bar_err;

//nitialize parameters

for $(u=0 ; u<8 ; u++)$

1

$\operatorname{len}[u]=99$;

off $[u]=99$;

alp $[u]=400$;

bet $[u]=400$;

the $[u]=400$;

l

// Determine config_rep

for( $\mathrm{u}=0 ; \mathrm{u}<$ (no_of_joints-1); $\mathrm{u++}$ )

I

if ( paramts[u][2] $==0)$

config_rep $[u]=1$; // Hayati representation

len[u] = paramts[u][0];

$\operatorname{alp}[u]=$ paramts $[u][2]^{*} \mathrm{pi} / 180$;

$\operatorname{bet}[\mathrm{u}]=0$;

the $[u]=\operatorname{paramts}[u][3]^{*} \mathrm{p} i / 180$;

$\operatorname{dlen}[u]=0$;

dalp $[u]=0$;

dbet $[u]=0$;

dthe $[u]=0$;

else

config_rep[u] $=0 ; / / \mathrm{DH}$ representation

$\operatorname{len}[u]=$ paramts[u][0];

off $[u]=$ paramts $[u][1]$;

alp $[u]=$ paramts[u][2]*pi/180;

the $[u]=$ paramts $[u][3]^{*} \mathrm{p} i / 180$;

dlen $[u]=0$;

$\operatorname{doff}[u]=0$;

dalp $[u]=0$; 


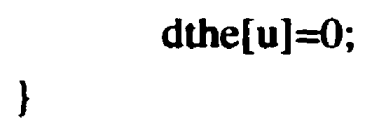

float det;

int $\mathrm{i}$;

det $A[0][0]^{*} \operatorname{minor} 3(A, 0,0)+A[0][1]^{*} \operatorname{minor} 3(A, 0,1)+A[0][2]^{*} \operatorname{minor} 3(A, 0,2)+A[0][3]^{*}=$ minor3 $(A, 0,3)$;

$B[0][0]=\operatorname{minor} 3(A, 0,0)$

$B[0][1]=\operatorname{minor} 3(A, 1,0)$;

$B[0][2]=\operatorname{minor} 3(A, 2,0)$;

$B[0][3]=\operatorname{minor} 3(A, 3,0)$; 


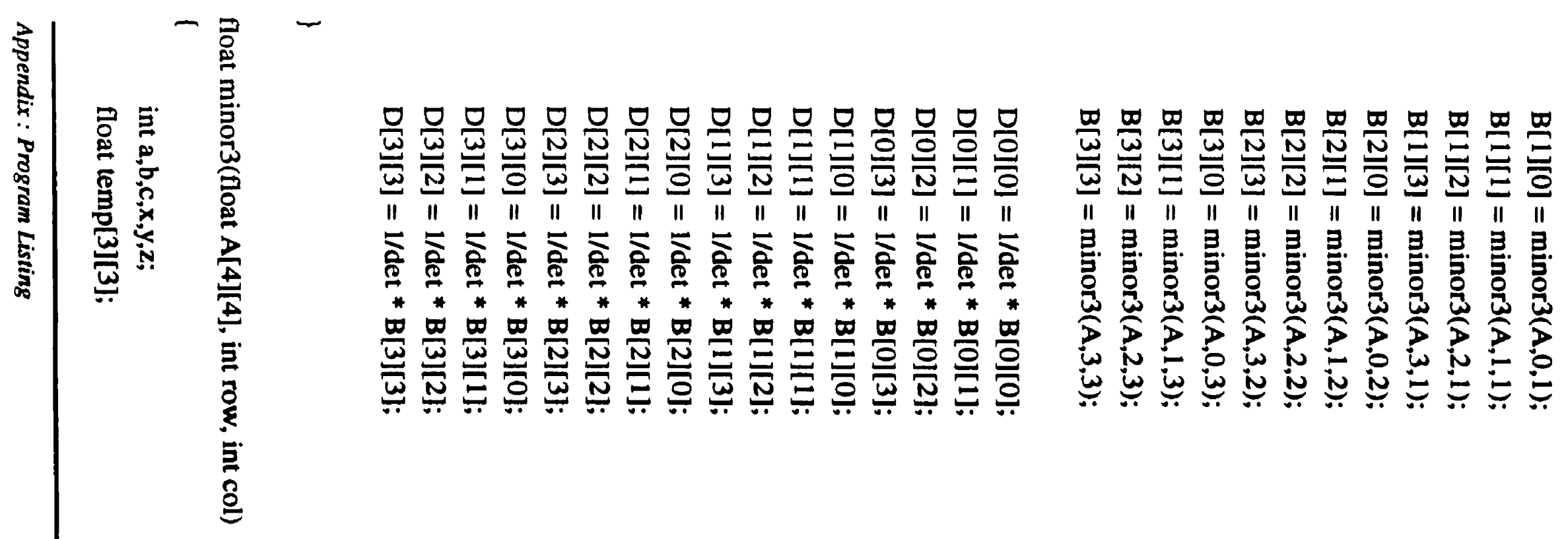




$$
\begin{aligned}
& \text { float det; } \\
& \begin{array}{r}
a=\text { row }+1 ; \\
\text { if }(a>=4) \\
a=a-4 ; \\
b=\text { row }+2 ; \\
\text { if }(b>=4) \\
\quad b=b-4 ; \\
c=\text { row }+3 ; \\
\text { if }(c>=4) \\
c=c-4 ;
\end{array}
\end{aligned}
$$

$\mathrm{x}=\mathrm{col}+1$;

if $(x>=4)$

$$
x=x-4 \text {; }
$$

$y=\mathrm{col}+2$;

if $(y>=4)$

$y=y-4$;

$\mathrm{z}=\mathrm{col}+3$;

if $(\mathrm{z}>=4)$

$$
z=z-4 \text {; }
$$

$$
\begin{aligned}
& \text { temp }[0][0]=\mathbf{A}[\mathbf{a}][\mathbf{x}] \text {; } \\
& \text { temp }[0][1]=A[a][y] ; \\
& \text { temp }[0][2]=\mathbf{A}[a][z] \text {; } \\
& \text { temp }[1][0]=\mathbf{A}[\mathrm{b}][\mathrm{x}] \text {; } \\
& \text { temp }[1][1]=A[b][y] ; \\
& \text { temp }[1][2]=A[b][z] ; \\
& \text { temp }[2][0]=A[c][x] \text {; } \\
& \text { temp }[2][1]=A[c][y] ; \\
& \text { temp }[2][2]=A[c][z] ;
\end{aligned}
$$

det

temp $[0][0]^{*} \operatorname{minor}(\operatorname{temp}, 0,0)+\operatorname{temp}[0][1] * \operatorname{minor}(\operatorname{temp}, 0,1)+\operatorname{temp}[0][2]{ }^{*} \operatorname{minor}(\operatorname{temp}$ $, 0,2)$;

if $((($ row + col $) \% 2)==1)$

$$
\operatorname{det}=-\operatorname{det} \text {; }
$$




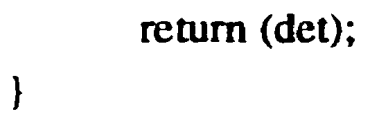


void multiply( float a[4][4], float b[4][4], float E[4][4])

l

int $r, c$;

for $(r=0 ; r<4 ; r++)$

l

for $(c=0 ; c<4 ; c++)$

I

$E[r][c]=a[r][0] * b[0][c]+a[r][1] * b[1][c]+a[r][2] * b[2][c]+a[r][3] * b[3][c] ;$

\}

1

)

float generate_K(float $K[8]$, float theta[8], int $w$ )

1

float $\mathbf{H}[8][4][4]$;

float DH[4][4];

float htemp 1[4][4],htemp2[4][4], rtemp1[4][4], rtemp2[4][4];

float pa, dp, dpl,bar_err;

float xi_1[3], yi_1[3], zi_l[3], xi[3], yi[3], zi[3], xih[3];

float di_1[3],di[3];

float Jbi[3],Jai[3],Jti[3],Jli[3],Jri[3];

int $\mathrm{u}$;

for $(\mathrm{u}=0 ; \mathrm{u}<\mathrm{no}$ of_joints; $\mathrm{u+})$

I

(

int $i, j$;

for $(i=0 ; i<4 ; i++)$

for $(j=0 ; j<4 ; j++)$

temp $[i][j]=t e m p 2[i][j]$;

\}

if (config_rep[u]= 1) // Hayati configuration

I

$H[u][0][0]=-\sin ($ alp $[u]) * \sin (\text { bet }[u])^{*} \sin ($ the $[u])+\cos (\operatorname{bet}[u]) * \cos ($ the $[u])$;

$H[u][0][1]=-\cos (\operatorname{alp}[u]) * \sin ($ the $[u]) ;$

$H[u][0][2]=\sin (\operatorname{alp}[u])^{*} \cos (\operatorname{bet}[u]) * \sin ($ the $[u])+\sin (b e t[u]) * \cos ($ the $[u])$; 


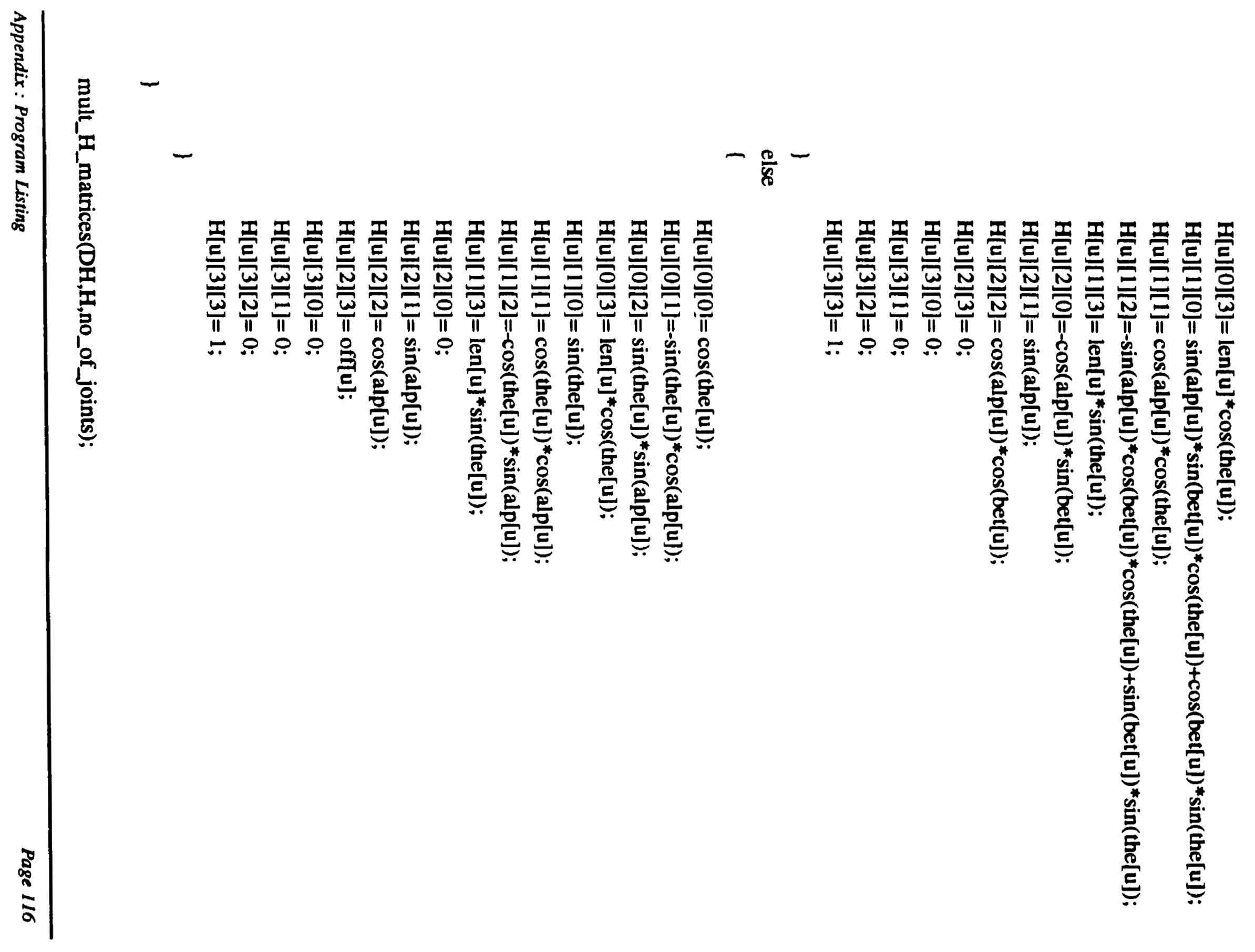




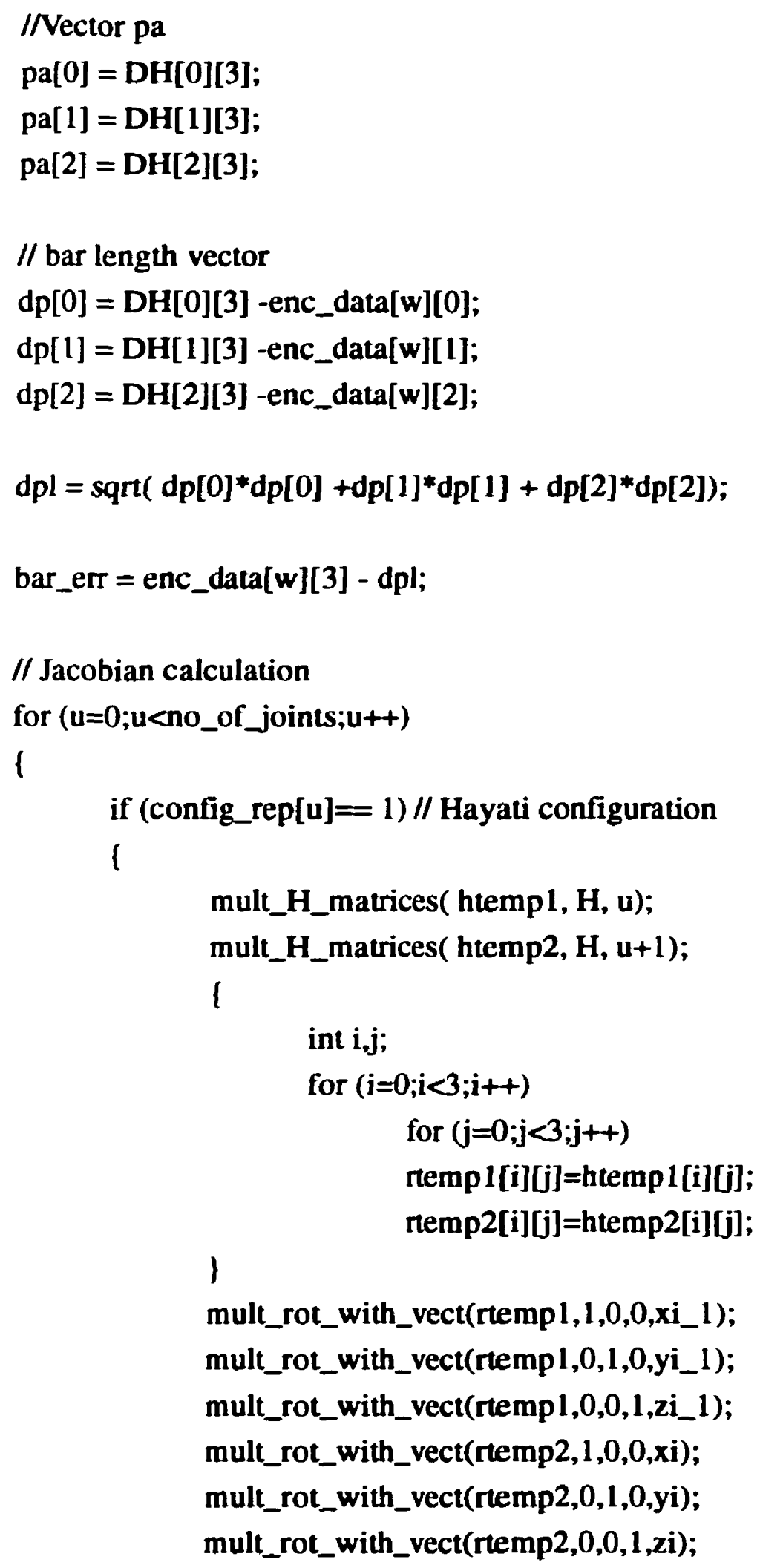




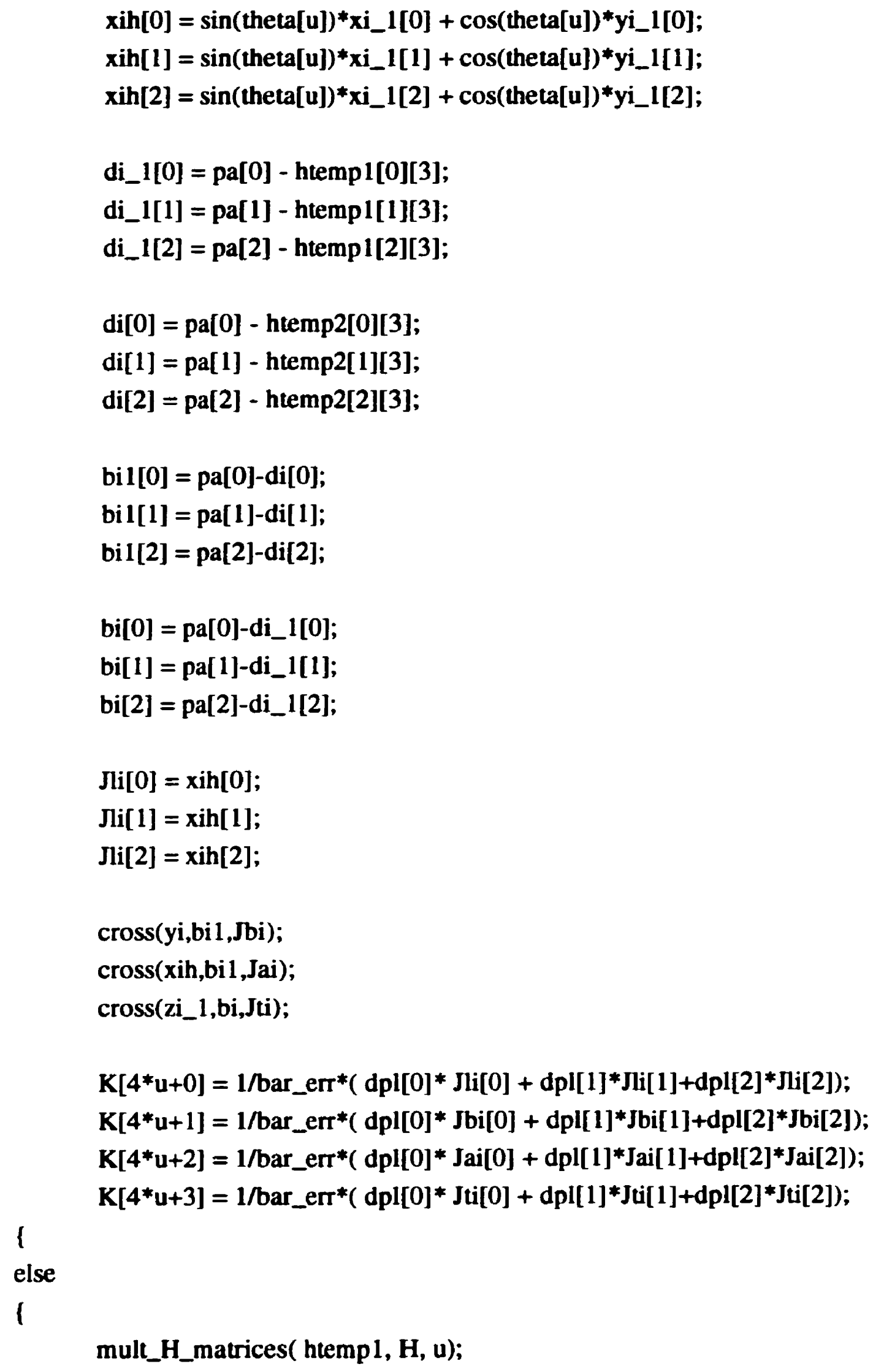

mult_H_matrices( htemp 1, H, u); 
mult_H_matrices( htemp2, $\mathrm{H}, \mathrm{u}+1$ );

I

int $i, j$;

for $(i=0 ; i<3 ; i++)$

for $(j=0 ; j<3 ; j++)$

rtemp 1 [i][j]=htemp 1 [i][j];

ntemp2[i][j]=htemp2[i][j];

)

mult_rot_with_vect(rtempl,1,0,0,xi_1);

mult_rot_with_vect(rtempl,0,1,0,yi_1);

mult_rot_with_vect(rtemp1,0,0,1,zi_1);

mult_rot_with_vect(rtemp2,1,0,0,xi);

mult_rot_with_vect(rtemp2,0,1,0,yi);

mult_rot_with_vect(rtemp2,0,0,1,zi);

di_1[0] = pa[0] - htemp $1[0][3]$;

di_1[1] = pa[1] - htemp $1[1][3]$;

di_1[2] = pa[2] - htemp 1[2][3];

$\operatorname{di}[0]=p a[0]-$ htemp2[0][3];

$\operatorname{di}[1]=\operatorname{pa}[1]-$ htemp2[1][3];

$\operatorname{di}[2]=p a[2]-$ htemp2[2][3];

bil[0] = pa[0]-di[0];

bil $[1]=\operatorname{pa}[1]-\operatorname{di}[1]$;

$\operatorname{bil}[2]=\operatorname{pa}[2]-\operatorname{di}[2]$;

bi[0] = pa[0]-di_1[0];

bi[1] = pa[1]-di_1[1];

bi[2] = pa[2]-di_1[2];

Ji[0] = xi[0];

$\mathrm{Jli}[1]=\mathrm{xi}[1]$;

$\operatorname{Jli}[2]=x i[2]$; 


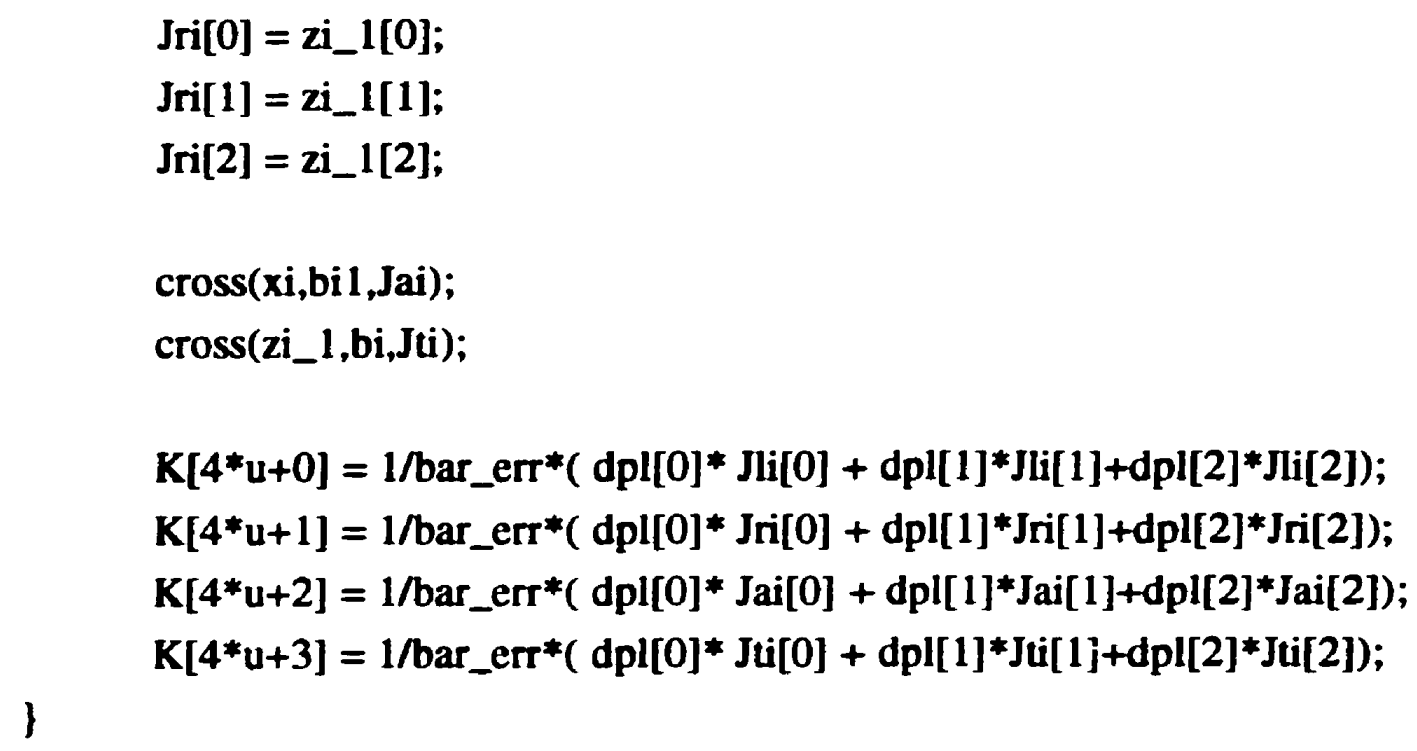

void mult_H_matrices ( float Hf[4][4],float $H[8][4][4]$, int nj)

l

float temp[4][4] $=\{(1,0,0,0\},\{0,1,0,0\},\{0,0,1,0\},\{0,0,0,1\}\}$;

float temp2 $[4][4]=\{\{1,0,0,0\},\{0,1,0,0\},\{0,0,1,0\},\{0,0,0,1\}\}$;

int $\mathbf{u}, \mathbf{v}, \mathbf{w}$;

for $(u=0 ; u<n j ; u++)$

1

for $(v=0 ; v<4 ; v++)$

for $(w=0 ; w<4 ; w++)$

temp[v][w]=temp2[v][w];

multiply(H[u],temp,temp2);

)

for $(v=0 ; v<4 ; v++)$

for $(w=0 ; w<4 ; w++)$

$H f[v][w]=t e m p 2[v][w]$;

\} 
void mult_rot_with_vect(float rtemp[3], int $i$, int $j$, int $k$, float res[3])

1 int $u$;

for $(u=0 ; u<3 ; u++)$

l

$$
\operatorname{res}[u]=\operatorname{rtemp}[0]^{* i}+\operatorname{rtemp}[1]^{* j}+\operatorname{rtemp}[2]^{* k}
$$

1

\}

void cross(float yi[3], float bi1[3], float Jbi[3])

l

$\operatorname{Jbi}[0]=\operatorname{yi}[1] * \operatorname{bil}[2]-\operatorname{yi}[2] * \operatorname{bil}[1] ;$

$\mathrm{Jbi}[1]=$ yi[2]*bil[0] - yi[0]*bil[2];

Jbi[2] = yi[0]*bil[1] - yi[1]*bil[0];

\} 